\title{
HYPERBOLIC LIMIT OF THE JIN-XIN RELAXATION MODEL
}

\author{
STEFANO BIANCHINI
}

AbStRaCt. We consider the special Jin-Xin relaxation model

$$
u_{t}+A(u) u_{x}=\epsilon\left(u_{x x}-u_{t t}\right)
$$

We assume that the initial data $\left(u_{0}, \epsilon u_{0}, t\right)$ are sufficiently smooth and close to $(\bar{u}, 0)$ in $L^{\infty}$ and have small total variation. Then we prove that there exists a solution $\left(u^{\epsilon}(t), \epsilon u_{t}^{\epsilon}(t)\right)$ with uniformly small total variation for all $t \geq 0$, and this solution depends Lipschitz continuously in the $L^{1}$ norm w.r.t. the initial data and time.

We then take the limit $\epsilon \rightarrow 0$, and show that $u^{\epsilon}(t)$ tends to a unique Lipschitz continuous semigroup $\mathcal{S}$ on a domain $\mathcal{D}$ containing the functions with small total variation and close to $\bar{u}$. The semigroup $\mathcal{S}$ defines a semigroup of relaxation limiting solutions to the quasilinear non conservative system

$$
u_{t}+A(u) u_{x}=0 .
$$

Moreover this semigroup coincides with the trajectory of a Riemann Semigroup, which is determined by the unique Riemann solver compatible with (0.1).

\section{Contents}

1. Introduction

2. Settings

2.1. Kinetic formulation

3.1. Lipschitz dependence w.r.t. $t$

3.2. Remarks about the structure of the Green kernel $\quad 15$

4. Center manifold of travelling profiles for relaxation $r$

4.1. Center manifold of travelling profiles $r$

4.2. Tangent vectors for travelling profiles in kinetic variables 18

5. Decomposition and source terms 19

5.1. Decomposition in travelling profiles $r$

5.2. Source terms of the components of the perturbation $h \quad 20$

5.3. Equations satisfied by $f, g \quad 25$

5.4. Simplification of the source terms $r$

6. Interaction estimates 28

$\begin{array}{lr}\text { 6.1. Opposite sign terms } & 28\end{array}$

$\begin{array}{lr}\text { 6.2. Transversal terms } & 28\end{array}$

6.3. Interaction terms of the same family 31

7. Conclusion of the proof of Theorem $1.1 \quad 37$

8. The hyperbolic limit $\epsilon \rightarrow 0 \quad 38$

8.1. Uniqueness of the limiting semigroup $\quad 40$

Appendix A. Explicit computations of the source terms for the perturbation $h \quad 41$

$\begin{array}{ll}\text { References } & 43\end{array}$

Date: August 30, 2004.

1991 Mathematics Subject Classification. 35L65.

Key words and phrases. Hyperbolic systems, conservation laws, relaxation, Jin-Xin model. 


\section{INTRODUCTION}

Consider the $n \times n$ hyperbolic system of conservation laws

$$
u_{t}+\mathcal{F}(u)_{x}=0, \quad u \in \mathbb{R}^{n} .
$$

Under the assumptions that the matrix $A(u)=D \mathcal{F}(u)$ is strict hyperbolicity and its eigenvalues are genuinely non linear or linearly degenerate, the global existence of a solution $u:[0,+\infty) \mapsto \mathbb{R}^{n}$ for small BV initial data has first been proved in [17].

The uniqueness of solutions and their Lipschitz dependence in $L^{1}$ w.r.t. the initial data has been established in a series of papers $[8,10,11,12,13,14]$. See $[9,16]$ for a general introduction to the theory of hyperbolic systems in one space dimension.

In the two papers [5, 3], a different approach has been used. Instead of constructing approximate solutions of the hyperbolic system (1.1) and studying their properties, the authors consider two approximations:

- vanishing viscosity approximation,

$$
u_{t}+\mathcal{F}(u)=\epsilon u_{x x}
$$

- semidiscrete upwind approximation,

$$
u_{t}(t, x)+\frac{1}{\epsilon}(\mathcal{F}(u(t, x))-\mathcal{F}(u(t, x-\epsilon)))=0 .
$$

The idea behind these schemes is that for $\epsilon>0$ the solution is clearly well defined, since both equations, when linearized, generate a continuous semigroup. Aim of the two papers $[5,3]$ is instead to prove that the solution of the two schemes (1.2), (1.3) satisfies some estimates which are independent on $\epsilon$. More precisely:

(1) the solution has uniformly bounded total variation for all $t \geq 0$, and its BV norm depends only on the BV norm of the initial data;

(2) the solution depends Lipschitz continuously on the initial data in $L^{1}$ and $t$.

It is easy to verify that both properties 1$), 2)$ are invariant for the hyperbolic rescaling $(t, x) \mapsto(t / \epsilon, x / \epsilon)$, and this rescaling allows us to put $\epsilon=1$ in (1.2), (1.3). As an example of some non scaling invariant property, note that the $L^{1}$ norm of a solution tend to 0 as $\epsilon \rightarrow 0$ : thus to obtain non trivial hyperbolic limits one has to assume $u \in L^{\infty}$, for example.

The advantage of this approach is that only the strictly hyperbolicity assumption on $A(u)$ is needed: as an example, the analysis of stability for a wave front tracking or Glimm scheme solution becomes quite complex without some assumptions on the structure of the solution, hence on the monotonicity of the eigenvalues. It is an open problem whether the results on hyperbolic systems obtained in $[5,3]$ can be proved directly at the hyperbolic level (1.1). We note also that in [1] it is shown how a similar approach cannot easily been extended to fully discrete schemes, e.g. Lax-Friedrichs or upwind Godunov scheme.

In the literature, there are other schemes used to approximate (1.1): the relaxation schemes. The easiest example is the scheme

$$
\left\{\begin{array}{ccc}
u_{t}+v_{x} & = & 0 \\
v_{t}+\Lambda^{2} u_{x} & = & \frac{1}{\epsilon}(\mathcal{F}(u)-v)
\end{array}\right.
$$

The above system is a special case of a class of relaxation systems introduced in [19], but we will refer to it as the Jin-Xin relaxation system from the name of the authors. For a general introduction and survey to relaxation schemes see [22].

At a formal level, one see that as $\epsilon \rightarrow 0$

$$
v=\mathcal{F}(u), \quad u_{t}+\mathcal{F}(u)_{x}=0 .
$$

As in the previous cases, the linearized version of (1.4) around $\bar{u}$ defines a continuous semigroup if $A(\bar{u})$ is hyperbolic and its eigenvalues $\lambda_{i}(\bar{u})$ satisfy

$$
-1+c \leq \lambda_{i}(\bar{u}) \leq 1-c, \quad c>0 .
$$

The transformation $x \mapsto x / \Lambda, v \mapsto \Lambda v$ allows us to set $\Lambda=1$ in (1.4), and with the hyperbolic rescaling $(t, x) \mapsto(t / \epsilon, x / \epsilon)$ we can take $\epsilon=1$ in (1.4). 
We should thus look for properties of the solution $(u, v)$ which are invariant under the hyperbolic rescaling.

By differentiating the second equation of (1.4) w.r.t. $x$ and using the first one obtains the nonlinear wave equation

$$
u_{t}+A(u) u_{x}=u_{x x}-u_{t t}
$$

with $A(u)=D \mathcal{F}(u)$. The above equation is meaningful also in the case $A(u)$ is not a Jacobian matrix, so that one cannot write a conservative form like (1.4).

In this paper we consider the wave equation (1.6), with $A(u)$ strictly hyperbolic but not necessarily a Jacobian of some vector function $\mathcal{F}(u)$. Our first main result is the following theorem:

Theorem 1.1. Consider the nonlinear wave equation (1.6), with $A(u)$ strictly hyperbolic and satisfying the stability conditions $(1.5)$ (with $\Lambda=1)$. Assume that the initial data $\left(u_{0}, u_{0, t}\right)$ are sufficiently smooth and with total variation less than $\delta_{1}$ :

$$
\left\|u_{0}\right\|_{L^{\infty}},\left\|u_{0, t}\right\|_{L^{\infty}} \leq \delta_{1},\left\|u_{0, x}\right\|_{L^{1}},\left\|u_{0, t x}\right\|_{L^{1}} \leq \delta_{1},\left\|\partial_{x}^{k} u_{0}\right\|_{L^{1}},\left\|\partial_{x}^{k} u_{0, t}\right\|_{L^{1}} \leq C^{\prime} \delta_{1}, \quad k=2,3 .
$$

for some constant $C^{\prime}$.

If $\delta_{1} \leq C^{-1} \delta_{0}$, with $C$ sufficiently large, then there exists a global solution $\left(u, u_{t}\right)$, defined for all $t \geq 0$, and with the $L^{1}$ norm of $u_{x}, u_{t x}$ less than $4 \delta_{0}$.

Moreover this solution depends continuously w.r.t. the initial data and time: for some constant $L$

$$
\begin{aligned}
& \left\|u(t)+e^{-t} u_{0, t}-\left(\hat{u}(s)+e^{-s} \hat{u}_{0, t}\right)\right\|_{L^{1}}+\left\|\left(u_{t}(t)-e^{-t} u_{0, t}\right)-\left(\hat{u}_{t}(s)-e^{-s} \hat{u}_{0, t}\right)\right\|_{L^{1}} \\
& \quad \leq L\left(|t-s|+\left\|\left(u_{0}+u_{0, t}\right)-\left(\hat{u}_{0}+\hat{u}_{0, t}\right)\right\|_{L^{1}}+\left\|u_{0, t x}-\hat{u}_{0, t x}\right\|_{L^{1}}+\left\|u_{0, t x x}-\hat{u}_{0, t x x}\right\|_{L^{1}}\right) .
\end{aligned}
$$

We note here that by means of the techniques used in this paper, one can avoid the assumption of smooth initial data: however to prove this result we need more technicalities, so will not consider this case here. Observe moreover that we can replace the first inequality of (1.7) by

$$
\left\|u_{0}-\bar{u}\right\|_{L^{\infty}},\left\|u_{0, t}\right\|_{L^{\infty}} \leq \delta_{1}
$$

and assume $A(u)$ strictly hyperbolic in a neighborhood of $\bar{u}$.

It is important to observe that the initial data are not assumed to have $u_{0, t} \in L^{1}$, which on the other hand is a natural condition for the initial data of (1.4), since $v_{x}=-u_{t}$. As we will show in the analysis, a part from exponentially decaying terms, $u_{t}$ becomes immediately in $L^{1}$. More precisely we will show that $u_{t}-e^{-t} u_{0, t}$ is integrable and has $L^{1}$ norm of the order of $4 \delta_{0}$.

Another observation is that since $u_{0, t} \notin L^{1}$, the assumption that $A(u)$ is conservative does not simplify too much the analysis. In fact in any case the second equation of (1.4) is meaningless. This is why we choose to state Theorem 1.1 in a non conservative setting.

Finally, notice that the Lipschitz dependence is w.r.t. the sum of $u_{0}+u_{0, t}$. This is clearly more precise that the dependence w.r.t. $u_{0}$ and $u_{0, t}$ separately. Moreover this dependence becomes meaningful in the hyperbolic limit $\epsilon \rightarrow 0$, see (1.12) below.

The second result is the analysis of the limit $\epsilon \rightarrow 0$. Denote by $u^{\epsilon}(t)$ the solution of the rescaled system

$$
u_{t}+A(u) u_{x}=\epsilon\left(u_{x x}-u_{t t}\right),
$$

and assume that the initial data are given by $\left(u_{0}, u_{0, t} / \epsilon\right)$, with $u_{0}, u_{0, t}$ fixed. This assumption on the form of $u_{t}(t=0)$ is needed in order to make the sum $u(t=0)+\epsilon u_{t}(t=0)$ to converge. We then prove the following theorem:

Theorem 1.2. Consider the nonlinear wave equation (1.9), with $A(u)$ strictly hyperbolic and satisfying the stability conditions $(1.5)$ (with $\Lambda=1$ ). Assume that the initial data $\left(u_{0}, \epsilon u_{0, t}\right)$ are sufficiently smooth and with total variation less that $\delta_{1}$ :

(1.10) $\left\|u_{0}\right\|_{L^{\infty}},\left\|u_{0, t}\right\|_{L^{\infty}} \leq \delta_{1},\left\|u_{0, x}\right\|_{L^{1}},\left\|\epsilon u_{0, t x}\right\|_{L^{1}} \leq \delta_{1}, \epsilon^{k}\left\|\partial_{x}^{k} u_{0}\right\|_{L^{1}}, \epsilon^{k+1}\left\|\partial_{x}^{k} u_{0, t}\right\|_{L^{1}} \leq C^{\prime} \delta_{1}, \quad k=2,3$.

for some constant $C^{\prime}$.

Then the solution $u^{\epsilon}(t)$ to $(1.9)$ converges in $L_{\mathrm{loc}}^{1}$ as $\epsilon \rightarrow 0$ to a unique limit $u(t)$. 
The $B V$ functions $u(t), t>0$, generates a Lipschitz continuous semigroup $u(t)=\mathcal{S}_{t} u(0)$ in $L_{\text {loc }}^{1}$ w.r.t. time and the initial data: for $t, s \geq \tau>0$

$$
\|u(t)-\hat{u}(s)\|_{L^{1}} \leq L\left(|t-s|+\|u(\tau)-\hat{u}(\tau)\|_{L^{1}}\right)
$$

Moreover, we have the estimate

$$
\left\|u(t)-\left(u_{0}+u_{0, t}\right)\right\|_{L^{1}} \leq L t,
$$

so that the correct initial data for $u(t)$ is given by $u_{0}+u_{t, 0}$.

This semigroup is defined on a domain $\mathcal{D}$ containing all the function with sufficiently small total variation, and can be uniquely identified by a relaxation limiting Riemann Solver, i.e. the unique Riemann solver compatible with (1.6).

We can thus say that the semigroup $\mathcal{S}$ defines a relaxation limiting solution to the quasilinear hyperbolic system

$$
u_{t}+A(u) u_{x}=0 .
$$

We repeat again that as a consequence of the above theorem, the right initial data for the limiting solution $u(t)$ is the sum of $u_{0}+u_{0, t}$. Thus $u(t)$ may have a jump at $t=0$, while for $t>0 u(t)$ is Lipschitz continuous w.r.t. $t$. For an easy example of why this happens, see Remark 2.2.

The paper is organized as follows.

In Section 2 we precise our assumptions on (1.6) to prove Theorem 1.1. In that section we also reformulate the equation for several variables: $u_{x}, u_{t}$, and the perturbation $h$ and its effective flux $\iota$. This reformulation (Section 2.1) is important because the $L^{1}$ stability can be proved in kinetic coordinates, which roughly speaking are given by the couples $\left(u_{x}+u_{t}, u_{x}-u_{t}\right)$ and $(h+\iota, h-\iota)$. The system these variables satisfy is of the form

$$
\left\{\begin{array}{l}
\omega_{t}^{-}-\omega_{x}^{-}=-\frac{I+A(u)}{2} \omega^{-}+\frac{I-A(u)}{2} \omega^{+}+s_{\omega}^{-}(t, x) \\
\omega_{t}^{+}+\omega_{x}^{+}=\frac{I+A(u)}{2} \omega^{-}-\frac{I-A(u)}{2} \omega^{+}++s_{\omega}^{+}(t, x)
\end{array}\right.
$$

i.e. we diagonalize the left hand side of (1.4).

The main problem is that $u_{t}$ is not in $L^{1}$, so that $\omega^{ \pm} \notin L^{1}$. We thus make the change of variable $\tilde{u}(t, x)=u(t, x)+e^{-t} u_{0, t}(x)$, recovering the estimates $\tilde{u}_{x}, \tilde{u}_{t} \in L^{1}$ (hence $\omega^{ \pm}(t=0) \in L^{1}$ ). A similar substitution (while more complicated) is done also for $h$ and $\iota$. The result of these computations is that by adding an exponential decaying in time term $s_{\omega}^{ \pm}$(or a slightly more general source term), we recover a $2 n \times 2 n$ kinetic system with initial data in $L^{1}$.

In Section 3 we prove some regularity estimates. These are needed to obtain the stability for $u_{t}$ in (1.8). The main result of this section is that if one assume that the initial data of a system of the form (1.14) is smooth and in $L^{1}$, then the solution can be constructed for a small forward interval of time and it has bounded $L^{1}$ norm; moreover also its derivatives are in $L^{1}$ in this interval of time, and can be estimated in terms of the $L^{1}$ norm of the solution (a part an exponential decaying term). The application of these results is that once we a priori assume the existence of an $L^{1}$ solution to (1.14) in the time interval $[0, T]$, then we have estimates on the $L^{1}$ norm of the derivatives. To conclude the section (Section 3.2), we show a refinement of the results on the Green kernel for general relaxations systems proved in [7, 23].

In Section 4 we extend the analysis of center manifold of travelling profiles to our relaxations case. It is not difficult to find $n$ center manifolds for (1.6), together with the conditions $u_{t}+\sigma u_{x}=0, \sigma_{x}=\sigma_{t}=0$, and $\sigma$ close to one eigenvalue $\lambda_{i}, i=1, \ldots, n$, of the matrix $A(u)$. The important point of this section (Section 4.2) is that we have 2 kinetic coordinate systems to identify the same travelling profile. Roughly speaking, both kinetic variables $u_{x}+u_{t}$ and $u_{x}-u_{t}$ can be used to describe every travelling profile. This gives us more freedom when decomposing a solution in travelling profiles.

In Section 5 we decompose our solution as a some of travelling profiles, and study the source terms. Using the fact that we have two sets of variable for every travelling profiles, i.e. we have $2 n$ possibilities, we decompose the solution $\left(u, u_{x}, u_{t}\right)$ as the sum of $2 n$ travelling profiles. The idea behind this decomposition can be more easily understood if we consider the variables $(u, v)$ of (1.4). In this conservative setting one can introduce the (primitive of the) kinetic variable $2 F^{ \pm}=u \pm v$. Then one tries to find for each $F^{ \pm} n$ travelling profiles which fit both $F_{x}^{ \pm}, F_{t}^{ \pm}$. Clearly the decomposition for $F^{-}$is independent on 
the decomposition for $F^{+}$, a part from the fact that we assume all travelling profiles to pass through $u=F^{-}+F^{+}$, since the nonlinearity depends only on $u$.

Using this decomposition also in our non conservative setting, we thus obtain the travelling profiles for $\left(u_{x}, u_{t}\right)$ and $(h, \iota)$. At this point one should write the equations for all these variables. We notice however that the structure of the kinetic systems is very similar, only some source term changes (due to the non conservative setting). We thus study just a prototype, i.e. the equations for the decomposition of the couple $(h, \iota)$ (Section 5.2), and write only which would be the equations for $\left(u_{x}, u_{t}\right)$ (Section 5.3).

We follow two approaches: one is just to substitute the decomposition in travelling profiles into the kinetic system and obtain the source terms. This is done in Appendix A. In the paper we prefer to follow a different approach, by showing that the form of the source terms follows by only knowing that our decomposition is exact (i.e. no source terms) on a single travelling profile. An important fact is that we are able to replace the differential condition on the speed $\sigma_{x}=\sigma_{t}=0$ with an algebraic condition: the speed of the travelling profile obtained from the decomposition of the kinetic variable $u_{x}+u_{t}$ is the same speed of the travelling profile for the decomposition of $u_{x}-u_{t}$.

We then conclude the section by rewriting the source term into a simplified form, which is more easily estimated. In Section 5.4 we also describe how we prove Theorem 1.1: we assume that in some interval $[0, T]$ the source terms are bounded by $2 \delta_{0}$ in $L^{1}([0, T] \times \mathbb{R})$, so that the solution $(h, \iota)\left(\right.$ or $\left.\left(u_{x}, u_{t}\right)\right)$ has $L^{1}(\mathbb{R})$ less than $4 \delta_{0} ; T>0$ because of the assumption on the initial data and the results of Section 3 . We then try to show that the $L^{1}([0, T] \times \mathbb{R})$ norm of source terms depends quadratically on the $L^{1}(\mathbb{R})$ norm of $(h, \iota)$. As a consequence if $\delta_{0} \ll 1$ the source is strictly less than $2 \delta_{0}$, hence the solution $(h, \iota)$ never reaches $4 \delta_{0}$ : we thus have $T=\infty$.

In Section 6 we prove that the source is bounded and quadratic. As in $[3,5]$, the source terms are divided into 4 categories:

(1) the scalar components of the travelling wave decomposition have opposite sign;

(2) interactions of waves of different families of travelling profiles;

(3) interactions of waves of the same family of travelling profiles;

(4) the speed of the travelling profiles of the $i$-th family is very different from the $i$-th eigenvalue $\lambda_{i}(u)$.

To each type of source term we associate a decreasing functional. The first two are already known: point 1) corresponds to the standard $L^{1}$ decay for $2 \times 2$ systems (Section 6.1, or [21]). Point 2) is the Relaxation Glimm functional introduced in [2]: in Section 6.2 we recall it and show how it is used to estimate the terms of point 2).

To estimates the term of point 3), in Section 6.3 we introduce a new functional, which formally corresponds to the Glimm interaction functional for waves of the same family for the Jin-Xin relaxation scheme. This functional is based on a new interpretation of the shortening curve functional of [6], see the beginning of Section 6.3.1. Point 4) correspond to the standard linear energy estimate extended to our non linear settings. We also show in Section 6.3.2 that an energy type estimate holds around travelling profiles.

In Section 7 we used all the results established so far to prove Theorem 1.1, following the proof outlined before.

In Section 8 we prove Theorem 1.2. Theorem 1.1 rewritten under the hyperbolic rescaling yields as $\epsilon \rightarrow 0$ a semigroup satisfying Theorem 1.2 a part from uniqueness. To prove uniqueness, we use the machinery of Viscosity Solutions and Riemann semigroup [8].

We thus recall that in $[4,5]$ it is proved that if BV estimates, Lipschitz dependence and finite speed of propagation are proved, then as $\epsilon \rightarrow 0$ the solution $u^{\epsilon}$ to (1.9) with initial data

$$
u_{0}(x)=\left\{\begin{array}{ll}
u^{-} & x<0 \\
u^{+} & x>0
\end{array} \quad u_{0, t}=0\right.
$$

converges to a unique self similar solution $u(t)$. We can say that there is a unique Riemann Solver compatible with (1.9). In Section 8.0.3 we recall how this Riemann Solver is constructed. The final part of this section (Section 8.1) is the proof that $u$ is a Viscosity Solution of the quasilinear hyperbolic system (1.13).

The paper is quite long, so we suggest here the most important results for a fast reading, avoiding possibly all technicalities, at least at first. 
Clearly, by assuming the system in conservation form and $u_{0, t} \in L^{1}$ (so that we have the variables $2 F^{ \pm}=u \pm v$ ), we can set $s_{\omega}^{ \pm}=0$ in (1.14), and replace $\omega^{ \pm}$with $f^{ \pm}=F_{x}^{ \pm}, g^{ \pm}=F_{t}^{ \pm}$to obtain the equations for the kinetic components of $\left(u_{x}, u_{t}\right)$. The only statements in Section 2 are thus the assumptions of strict hyperbolicity, non degeneracy and smoothness of the initial data.

While one can just believe in the classic regularity results on semigroups of Section 3, the computations in the center manifold of travelling profiles of Section 4 are important, over all the changes of coordinates of Section 4.2. All the rest of the paper is in fact based upon them.

Of Section 5, it is important to see the decomposition in travelling profiles and the relations among the various kinetic components, Section 5.1. Moreover, the first part of Section 5.2 gives the main ideas on the form of the source terms: we believe this is one of the most important part. The effective computations are just a technical stuff, one can jump to (5.24).

The results contained in Section 6 are important, since several nonlinear functionals are introduced. The new ones are in Section 6.3: we believe these computations important too, and in particular Example 6.2 should be illuminating.

The remaining results (uniqueness of the hyperbolic limit) is in some sense the relaxation version of results already knows for vanishing viscosity and semidiscrete schemes. A longer analysis can be find in $[3,5]$.

\section{Settings}

Consider the $n \times n$ hyperbolic system

$$
u_{t}+A(u) u_{x}=u_{x x}-u_{t t},
$$

with $u, v \in \mathbb{R}^{n}, A(u): \mathbb{R}^{n} \mapsto \mathbb{R}^{n \times n}$ sufficiently smooth function. Denote with $r_{i}(u), l_{i}(u), \lambda_{i}(u)$, $i=1, \ldots, n$, the right, left eigenvectors, eigenvalues of $A(u)$, normalized such that

$$
A(u) r_{i}(u)=\lambda_{i}(u) r_{i}(u), \quad\left|r_{i}(u)\right|=1, \quad\left\langle l_{j}(u), r_{i}(u)\right\rangle= \begin{cases}1 & j=i \\ 0 & j \neq i\end{cases}
$$

For stability of the model near the equilibrium point $\bar{u}=0$, we require that there is a $c>0$ so that

$$
-1+3 c \leq \lambda_{1}(0), \quad \lambda_{n}(0) \leq 1-3 c, \quad \lambda_{i+1}(0)-\lambda_{i}(0) \geq 3 c, \quad i=1, \ldots, n-1 .
$$

As we observe in the introduction, a particular case is when the system (2.1) is in conservation form, i.e. $A(u)=D \mathcal{F}(u)$, with $\mathcal{F}: \mathbb{R}^{n} \mapsto \mathbb{R}^{n}$ : by defining $v_{x}=-u_{t}$ (and assuming the last in $L^{1}$ ) we recover the relaxation model (1.4) with $\Lambda=\epsilon=1$,

$$
\left\{\begin{array}{llc}
u_{t}+v_{x} & = & 0 \\
v_{t}+u_{x} & = & \mathcal{F}(u)-v
\end{array}\right.
$$

The above system can be rewritten in kinetic form as

$$
\left\{\begin{array}{l}
F_{t}^{-}-F_{x}^{-}=M^{-}(u)-F^{-} \\
F_{t}^{+}+F_{x}^{+}=M^{+}(u)-F^{+}
\end{array}\right.
$$

where

$$
u=F^{+}+F^{-}, \quad v=F^{+}-F^{-}, \quad M^{-}(u)=\frac{u-\mathcal{F}(u)}{2}, \quad M^{+}(u)=\frac{u+\mathcal{F}(u)}{2}
$$

The function $M^{-}(u), M^{+}(u)$ are called Maxwellians, and by assumptions (2.3) we have that

$$
M^{-}(u)+M^{+}(u)=u, \quad M^{+}(u)-M^{-}(u)=\mathcal{F}(u), \quad D M^{-}(u), D M^{+}(u)>0, \quad u \in B\left(0,5 \delta_{0}\right),
$$

where $\delta_{0}$ is a sufficiently small constant.

The initial data $u_{0}, u_{0, t}$ of the system (2.1) takes values in a neighborhood of $(0,0)$ and have small total variation,

$$
\left\|u_{0}\right\|_{L^{\infty}},\left\|u_{0, t}\right\|_{L^{\infty}} \leq \delta_{1}, \quad \text { Tot.Var. }\left(u_{0}\right), \operatorname{Tot} . \operatorname{Var} .\left(u_{0, t}\right) \leq \delta_{1} .
$$

To simplify the analysis and have more concise statements, we require some regularity on $u_{0}, u_{0, t}$, namely

$$
\left\|u_{0, x x}\right\|_{L^{1}},\left\|u_{0, t x x}\right\|_{L^{1}},\left\|u_{0, x x x}\right\|_{L^{1}},\left\|u_{0, t x x x}\right\|_{L^{1}} \leq C^{\prime} \delta_{1},
$$

for some constant $C^{\prime}$. 
From (2.3) it follows that for $u \in B\left(0,5 \delta_{0}\right)$

$$
\min _{i, u v} \lambda_{i+1}(u)-\lambda_{i}(v) \geq 2 c>0, \quad \min _{u} \lambda_{1}(u) \geq-1+2 c, \quad \max _{u} \lambda_{n}(u) \geq-1+2 c .
$$

Remark 2.1. These regularity assumption can be relaxed requiring only $u_{0}, u_{0, t} \in \mathrm{BV}$, but the analysis of more general initial data add some technicalities, which are not essential to our problem. In fact, by means of the result on the Green kernel $\Gamma$ of the linearized problem around $u=0$, see Section 3 , one can split the solution into two parts:

- an exponential time decaying part which collects the "non smooth" initial data. This part has a finite BV norm;

- the smooth part, which can be studied as we do in what follows for smooth initial data. The only difference is that there will be a source term proportional to the non smooth part, exponentially decaying in time.

However such a general initial data is in some sense artificial: the idea behind these schemes is to recover the non smooth solution of the limiting hyperbolic system

$$
u_{t}+A(u) u_{x}=0
$$

as the limit of smooth to (2.1) using the hyperbolic rescaling $(t, x) \mapsto(t / \epsilon, x / \epsilon)$. We just add these remark to underline which can be the most general setting of to have BV solutions.

Aim of the next part is to find a convenient formulation for (2.1). This formulation is essentially the coordinates where one can prove $L^{1}$ contraction for the $2 \times 2$ case. We call it kinetic formulation.

2.1. Kinetic formulation. To prove BV bounds and Lipschitz stability, we need to consider several variables:

(1) the derivatives $u_{t}, u_{x}$;

(2) the perturbations $h$ and its effective flux $\iota$.

Along with these variables we will consider also their kinetic counterparts, obtained with a procedure similar to the one leading from (2.1) to (2.5). However, since the system is not in conservation form, and $u_{t} \notin L^{1}$, the kinetic variables (which in the conservative case are just the $x$ derivatives of $F^{ \pm}$)

$$
f^{ \pm}=\frac{1}{2}\left(u_{x} \pm u_{t}\right)
$$

are in general not in $L^{1}$. One can observe however that the non integrable part in $u_{t}$ decays exponentially in time, so that we recover the case $f^{ \pm} \in L^{1}$, i.e. $u_{t} \in L^{1}$. Define in fact

$$
\tilde{u}(t, x)=u(t, x)+e^{-t} u_{0, t}(x) .
$$

Then we have that (2.1) becomes

$$
\tilde{u}_{t}+A\left(\tilde{u}-e^{-t} u_{0, t}\right) \tilde{u}_{x}=\tilde{u}_{x x}-\tilde{u}_{t t}+e^{-t}\left(A\left(\tilde{u}-e^{-t} u_{0, t}\right) u_{0, t x}-u_{0, t x x}\right),
$$

with initial data

$$
\tilde{u}_{0}=u_{0}+u_{0, t}, \quad \tilde{u}_{0, t}=0 .
$$

The last term, by means of $(2.8),(2.9)$, is in $L^{1}\left(\mathbb{R}^{+} \times \mathbb{R}\right)$ and has norm $C^{\prime}\left(\|A\|_{L^{\infty}}+1\right) \delta_{1}$. A more refined decomposition is given by

$$
\tilde{u}(t, x)=u(t, x)+e^{-t}\left\langle l_{i}(0), u_{0, t}\left(x-\lambda_{i}(0) t\right)\right\rangle r_{i}(0)=u(t, x)+e^{-t} u_{0 i, t}\left(x-\lambda_{i}(0) t\right),
$$

(essentially we are following the waves with speed $\lambda_{i}$ ) to which it corresponds the equation

$$
\tilde{u}_{t}+A(u) \tilde{u}_{x}=\tilde{u}_{x x}-\tilde{u}_{t t}+e^{-t}(A(u)-A(0)) u_{0 i, t x}-e^{-t}\left(1-\lambda_{i}^{2}(0)\right) u_{0 i, t x x},
$$

with initial data given by

$$
\tilde{u}_{0}=u_{0}+u_{0, t}, \quad \tilde{u}_{0, t}=-\lambda_{i}(0) u_{0 i, t x} .
$$

The source term in the right hand side is of order $C \delta_{0} \delta_{1}$, with $C$ depending only on the size of the neighborhood $B\left(0,5 \delta_{0}\right)$. Observe that now $\tilde{u}_{0, t} \in L^{1}(\mathbb{R})$. 
To keep the computations as easy as possible, we will use the simpler decomposition (2.11). This will (in some sense) give weight of order $C \delta_{1}$ to the ( $x$ derivatives of the) initial data $u_{0, t}$, but for our purpose (small initial data $\delta_{1} \leq C^{-1} \delta_{0}$ ) this decomposition will be sufficient.

Before writing the equations for the kinetic components, we observe that a source term in the equation

$$
\omega_{t}+(A(u) \omega)_{x}=\omega_{x x}-\omega_{t t}+s_{1, x}(t, x)+s_{2, t}(t, x)+s_{2}(t, x),
$$

can be represented in kinetic coordinates

$$
\omega=\omega^{-}+\omega^{+}, \quad \int_{-\infty}^{x}\left(\omega_{t}(t, y)-s_{2}(t, y)\right) d y=\omega^{-}-\omega^{+}
$$

as

$$
\left\{\begin{array}{l}
\omega_{t}^{-}-\omega_{x}^{-}=-\frac{I+A(u)}{2} \omega^{-}+\frac{I-A(u)}{2} \omega^{+}-\frac{1}{2} s_{1}(t, x)+\frac{1}{2} s_{2}(t, x) \\
\omega_{t}^{+}+\omega_{x}^{+}=\frac{I+A(u)}{2} \omega^{-}-\frac{I-A(u)}{2} \omega^{+}+\frac{1}{2} s_{1}(t, x)+\frac{1}{2} s_{2}(t, x)
\end{array}\right.
$$

In other words, if $\Gamma(t, x ; s, y)=\left(\Gamma_{1}, \Gamma_{2}\right)$ is the Green kernel of the perturbed wave equation (2.15) with initial data $\left(\omega, \omega_{t}\right)$, then we can write (see Section 3 for more details on $\Gamma$ )

$$
\begin{aligned}
\omega(t, x)= & \int_{\mathbb{R}}\left(\Gamma_{1}(t, x ; 0, y) \omega(0, y)+\Gamma_{2}(t, x ; 0, y) \omega_{t}(0, y)\right) d y \\
& +\int_{0}^{t} \int_{\mathbb{R}}\left(\Gamma_{1}(t, x ; s, y) s_{2}(s, y)-\Gamma_{2}(t, x ; s, y) s_{1, x}(s, y)\right) d y .
\end{aligned}
$$

This means that $-s_{1, x}$ is interpreted as an initial data for the time derivative $\omega_{t}$, and $s_{2}$ is the initial data for $\omega$. Since all the estimates we are going to consider (in particular the conservation of $L^{1}$ norm) are written in terms of the kinetic components, we will always try to decompose the source terms as in (2.16). Note that by writing

$$
s_{2}(t, x)=\int_{0}^{t} e^{\tau-t} s(\tau, x) d \tau
$$

we have $s_{2, t}+s_{2}=s$, so that by convolution in time with an integrable kernel we can write any source term in the form $s_{2, t}+s_{2}$. Depending on the form of the source terms $s_{1}, s_{2}$, we will choose the most convenient way.

2.1.1. Equations for $f^{-}, f^{+}$. We look for the equations for the kinetic variables

$$
f^{-}=\frac{\tilde{u}_{x}+\tilde{u}_{t}}{2}, \quad f^{+}=\frac{\tilde{u}_{x}-\tilde{u}_{t}}{2},
$$

which (after noticing that the source term is the $x$ derivative $s_{1, x}$, with the notation (2.15)) are

$$
\left\{\begin{array}{l}
f_{t}^{-}-f_{x}^{-}=-\frac{I+A(u)}{2} f^{-}+\frac{I-A(u)}{2} f^{+}-s_{f}(t, x) \\
f_{t}^{+}+f_{x}^{+}=\frac{I+A^{(}(u)}{2} f^{-}-\frac{I-A^{(}(u)}{2} f^{+}+s_{f}(t, x)
\end{array}\right.
$$

with initial data

$$
f^{-}(0, x)=\frac{1}{2}\left(u_{0, x}+u_{0, t x}\right), \quad f^{+}(0, x)=\frac{1}{2}\left(u_{0, x}+u_{0, t x}\right)
$$

and source term

$$
s_{f}(t, x)=\frac{e^{-t}}{2}\left(A(u) u_{0, t x}-u_{0, t x x}\right) .
$$

The initial data are now in $L^{1}$ and the source is bounded in $L^{1}$ by

$$
\int_{\mathbb{R}}\left|s_{f}(t, x)\right| d x \leq C \delta_{1} e^{-t}, \quad \int_{\mathbb{R}}\left|s_{f, x}(t, x)\right| d x \leq C \delta_{1} e^{-t} .
$$


2.1.2. Equation for $g^{-}, g^{+}$. To decompose $f^{-}, f^{+}$in travelling waves, we have to study the equations for the their effective fluxes. In the conservative case, i.e. when $\left(F^{-}, F^{+}\right)$are well defined, so that $f^{-}=F_{x}^{-}$, $f^{+}=F_{x}^{+}$, we would study the equations for $g^{-}=F_{t}^{-}, g^{+}=F_{t}^{+}$.

In our general non conservative case, $\tilde{u}_{t}$ is well defined and at $t=0$ is in $L^{1}$, and we use the same procedure for $u_{x}$ to replace the initial data $\int_{-\infty}^{x} \tilde{u}_{t t} d y \in L^{\infty}$ with an integrable function.

Differentiating (2.13) w.r.t. $t$ we obtain that the equation for $\tilde{u}_{t}$ is

$$
\begin{aligned}
\left(\tilde{u}_{t}\right)_{t}+\left(A(u) \tilde{u}_{t}\right)_{x}= & \left(\tilde{u}_{t}\right)_{x x}-\left(\tilde{u}_{t}\right)_{t t}+D A\left(u_{x} \times \tilde{u}_{t}-u_{t} \times \tilde{u}_{x}\right)+\left(e^{-t}\left(A(u) u_{0, t x}-u_{0, t x x}\right)\right)_{t} \\
= & \left(\tilde{u}_{t}\right)_{x x}-\left(\tilde{u}_{t}\right)_{t t}+D A\left(\tilde{u}_{x} \times \tilde{u}_{t}-\tilde{u}_{t} \times \tilde{u}_{x}\right) \\
& -e^{-t} D A(u)\left(u_{0, t x} \times \tilde{u}_{t}+u_{0, t} \times \tilde{u}_{x}\right)+\left(e^{-t}\left(A(u) u_{0, t x}-u_{0, t x x}\right)\right)_{t} \\
= & \left(\tilde{u}_{t}\right)_{x x}-\left(\tilde{u}_{t}\right)_{t t}+s_{\tilde{u}_{t}}(t, x)
\end{aligned}
$$

with initial data

$$
\tilde{u}_{t}(0, x)=0, \quad \tilde{u}_{t t}(0, x)=u_{0, t t}+u_{0, t}=u_{0, x x}-A\left(u_{0}\right) u_{0, x} .
$$

Note that $\tilde{u}_{t t}(0)$ is in $L^{1}$, but not its integral in $x$.

We associate to (2.24) the kinetic model

$$
\left\{\begin{array}{l}
\left(\tilde{u}_{t}\right)_{t}+p_{x}=\int_{0}^{t} e^{\tau-t} s_{\tilde{u}_{t}}(\tau, x) d \tau \\
p_{t}+\left(\tilde{u}_{t}\right)_{x}=A(u) \tilde{u}_{t}-p
\end{array}\right.
$$

with $p$ obtained by integrating the first equation in $x$ (in the language of (2.17), we associate the source $s_{\tilde{u}_{t}}$ to $\tilde{u}_{t}$ ). Note that $p$ is well defined in $L^{\infty}$, since both $\tilde{u}_{t}$ that the source $s_{\tilde{u}_{t}}$ are in $L^{1}$ (at least for small t). A major problem here is that the initial data for $p$ is only in $L^{\infty}$ (at least the part $\int_{\infty}^{x} A\left(u_{0}\right) u_{0, x} d y$ ), so that the kinetic variables $g^{ \pm}=\left(\tilde{u}_{t} \pm p\right) / 2$ are not in $L^{1}$.

We replace now $p$ with $\tilde{p}$ given by

$$
p(t, x)=\tilde{p}(t, x)+e^{-t} \int_{-\infty}^{x} A\left(u_{0}(y)\right) u_{0, x}(y) d y,
$$

so that we obtain the Jin-Xin scheme

$$
\left\{\begin{array}{ccc}
\left(\tilde{u}_{t}\right)_{t}+\tilde{p}_{x} & = & -e^{-t} A\left(u_{0}\right) u_{0, x}(x)+\int_{0}^{t} e^{-(t-\tau)} s_{\tilde{u}_{t}}(\tau, x) d \tau \\
\tilde{p}_{t}+\left(\tilde{u}_{t}\right)_{x} & = & A(u) \tilde{u}_{t}-\tilde{p}
\end{array}\right.
$$

with initial data

$$
\tilde{u}_{t}(0, x)=0, \quad \tilde{p}(0, x)=-u_{0, x} .
$$

Now both the initial data are in $L^{1}$, so that kinetic model for $\tilde{u}_{t}=g^{+}+g^{-}, \tilde{p}=g^{+}-g^{-}$takes the form

$$
\left\{\begin{array}{l}
g_{t}^{-}-g_{x}^{-}=-\frac{I+A(u)}{2} g^{-}+\frac{I-A(u)}{2} g^{+}+s_{g}(t, x) \\
g_{t}^{+}+g_{x}^{+}=\frac{I+A(u)}{2} g^{-}-\frac{I-A(u)}{2} g^{+}+s_{g}(t, x)
\end{array}\right.
$$

with initial data

and source

$$
g^{-}(0, x)=\frac{u_{0, x}}{2}, \quad g^{+}(0, x)=-\frac{u_{0, x}}{2},
$$

$$
\begin{aligned}
s_{g}(t, x)= & -\frac{e^{-t}}{2} A\left(u_{0}\right) u_{0, x}(x)+\frac{1}{2} \int_{0}^{t} e^{-(t-\tau)} s_{\tilde{u}_{t}}(\tau, x) d \tau \\
= & \frac{e^{-t}}{2}\left(\left(A(u)-A\left(u_{0}\right)\right) u_{0, t x}-A\left(u_{0}\right) u_{0, x}\right)+\frac{1}{2} \int_{0}^{t} e^{\tau-t} D A\left(\tilde{u}_{x} \times \tilde{u}_{t}-\tilde{u}_{t} \times \tilde{u}_{x}\right)(\tau) d \tau \\
& -\frac{e^{-t}}{2} \int_{0}^{t} D A(u)\left(u_{0, t x} \times \tilde{u}_{t}+u_{0, t} \times \tilde{u}_{x}\right) d \tau-\frac{e^{-t}}{2} \int_{0}^{t}\left(A(u) u_{0, t x}-u_{0, t x x}\right) d \tau .
\end{aligned}
$$

This source term can be divided into 3 parts:

(1) exponentially decaying terms of order $C \delta_{1}$,

$$
\frac{e^{-t}}{2}\left(\left(A(u)-A\left(u_{0}\right)\right) u_{0, t x}(x)-A\left(u_{0}\right) u_{0, x}(x)\right)-\frac{e^{-t}}{2} \int_{0}^{t}\left(A(u) u_{0, t x}-u_{0, t x x}\right) d \tau ;
$$


(2) exponentially decaying terms of order $\sup _{\tau \in[0, t]}\left(\left\|\tilde{u}_{x}(\tau)\right\|_{L^{1}}+\left\|\tilde{u}_{t}(\tau)\right\|_{L^{1}}\right) \delta_{1} e^{-t / 2}$,

$$
-\frac{e^{-t}}{2} \int_{0}^{t} D A(u)\left(u_{0, t x} \times \tilde{u}_{t}+u_{0, t} \times \tilde{u}_{x}\right) d \tau
$$

(3) a non local in time interaction term

$$
\frac{1}{2} \int_{0}^{t} e^{\tau-t} D A\left(\tilde{u}_{x} \times \tilde{u}_{t}-\tilde{u}_{t} \times \tilde{u}_{x}\right)(\tau) d \tau .
$$

We conclude that if we the solution exists in the time interval $[0, T]$

$$
\left\|s_{g}(t)\right\|_{L^{1}} \leq C\left(1+\sup _{\tau \in[0, t]}\left(\left\|\tilde{u}_{t}\right\|_{L^{1}}+\left\|\tilde{u}_{t}\right\|_{L^{1}}\right)\right) \delta_{1} e^{-t / 2}+C \sup _{\tau \in[0, t]}\left(\left\|\tilde{u}_{x x}(\tau)\right\|_{L^{1}}\left\|\tilde{u}_{t}(\tau)\right\|_{L^{1}}\right),
$$

$$
\left\|s_{g, x}(t)\right\|_{L^{1}} \leq C\left(1+\sup _{\tau \in[0, t]}\left(\left\|\tilde{u}_{t, x}(\tau)\right\|_{L^{1}}+\left\|\tilde{u}_{t, x}(\tau)\right\|_{L^{1}}\right)\right) \delta_{1} e^{-t / 2}+C \sup _{\tau \in[0, t]}\left(\left\|\tilde{u}_{x x}(\tau)\right\|_{L^{1}}\left\|\tilde{u}_{t x}(\tau)\right\|_{L^{1}}\right) .
$$

Observe that by contruction we have

$$
\tilde{u}_{t}=g^{+}+g^{-}=f^{-}-f^{+} .
$$

This is an important relation among the two couples of variables $\left(f^{-}, f^{+}\right)$and $\left(g^{-}, g^{+}\right)$, which will be used in Section 5.1.

2.1.3. Equations for $h^{-}, h^{+}$and $\iota^{-}, \iota^{+}$. The computations for $h$ are very similar to the ones for $g$. By taking an infinitesimal perturbation of (2.1) we obtain the equation

$$
h_{t}+(A(u) h)_{x}=h_{x x}-h_{t t}+D A(u)\left(u_{x} \times h-h \times u_{x}\right)
$$

Here the initial data are assumed to be $h_{0}, h_{0, t}$ in $L^{1}(\mathbb{R})$ small, i.e. satisfying (2.8), (2.9), replacing $u_{x}$ with $h$,

$$
\left\|h_{0, x}\right\|_{L^{1}},\left\|h_{0, t x}\right\|_{L^{1}},\left\|h_{0, x x}\right\|_{L^{1}},\left\|h_{0, t x x}\right\|_{L^{1}} \leq C^{\prime} \delta_{1} .
$$

Clearly since the equation for $h$ is linear this is not a restriction.

We first replace $h$ with $\tilde{h}=h+e^{-t} h_{0, t}$ (as before this is not the smartest substitution), obtaining the equation

$$
\tilde{h}_{t}+(A(u) \tilde{h})_{x}=\tilde{h}_{x x}-\tilde{h}_{t t}+e^{-t}\left(A(u) h_{0, t x}-h_{0, t x x}\right)+D A(u)\left(u_{x} \times \tilde{h}-h \times u_{x}\right) .
$$

with initial data

$$
\tilde{h}(0, x)=h_{0}+h_{0, t}, \quad \tilde{h}_{t}(0, x)=0 .
$$

By defining $\tilde{\iota}$ by the Jin-Xin system

$$
\left\{\begin{array}{ccc}
\tilde{h}_{t}+(-\tilde{\iota})_{x} & =e^{-t} \int_{0}^{t}\left(\left(A(u) h_{0, t x}-h_{0, t x x}\right)+e^{\tau} D A(u)\left(u_{x} \times \tilde{h}-h \times u_{x}\right)\right) d \tau \\
(-\tilde{\iota})_{t}+\tilde{h}_{x} & = & A(u) \tilde{h}-(-\tilde{\iota})
\end{array}\right.
$$

we obtain the kinetic model for $\tilde{h}=h^{-}+h^{+}, \tilde{\iota}=h^{-}-h^{+}$:

$$
\left\{\begin{array}{l}
h_{t}^{-}-h_{x}^{-}=-\frac{I+A(u)}{2} h^{-}+\frac{I-A(u)}{2} h^{+}+s_{h}(t, x) \\
h_{t}^{+}+h_{x}^{+}=\frac{I+A(u)}{2} h^{-}-\frac{\left.I-A^{(} u\right)}{2} h^{+}+s_{h}(t, x)
\end{array}\right.
$$

with initial data $h^{-}=h^{+}=\left(h_{0}+h_{0, t}\right) / 2$ and source term

$$
s_{h}(t, x)=\frac{e^{-t}}{2} \int_{0}^{t}\left(\left(A(u) h_{0, t x}-h_{0, t x x}\right)+e^{\tau} D A(u)\left(u_{x} \times \tilde{h}-h \times u_{x}\right)\right) d \tau
$$

$$
\left\|s_{h}(t)\right\|_{L^{1}} \leq C\left(1+\sup _{\tau \in[0, t]}\left(\left\|\tilde{u}_{x}(\tau)\right\|_{L^{1}}+\|\tilde{h}(\tau)\|_{L^{1}}\right)\right) \delta_{1} e^{-t / 2}+C \sup _{\tau \in[0, t]}\left(\left\|\tilde{u}_{x x}(\tau)\right\|_{L^{1}}\|\tilde{h}(\tau)\|_{L^{1}}\right),
$$

$(2.41)\left\|s_{h, x}(t)\right\|_{L^{1}} \leq C\left(1+\sup _{\tau \in[0, t]}\left(\left\|\tilde{u}_{x x}(\tau)\right\|_{L^{1}}+\left\|\tilde{h}_{x}(\tau)\right\|_{L^{1}}\right)\right) \delta_{1} e^{-t / 2}+C \sup _{\tau \in[0, t]}\left(\left\|\tilde{u}_{x x}(\tau)\right\|_{L^{1}}\left\|\tilde{h}_{x}(\tau)\right\|_{L^{1}}\right)$.

We have used (2.35). 
We finally compute the equations satisfied by $\tilde{\iota}$ (which are the most complicated ones, as one may expect). By the first equation of system (2.38) we have that

$$
\tilde{\iota}_{x}=\tilde{h}_{t}-2 s_{h}(t, x),
$$

and the substitution of this relation into the time derivative of the second yields

$$
\begin{aligned}
0 & =\tilde{\iota}_{t}+\tilde{\iota}_{t t}-\tilde{h}_{t x}+(A(u) \tilde{h})_{t} \\
& =\tilde{\iota}_{t}+\tilde{\iota}_{t t}-\tilde{\iota}_{x x}+(A(u) \tilde{\iota})_{x}-2 s_{h, x}+D A(u)\left(u_{t} \times \tilde{h}-u_{x} \times \tilde{\iota}\right)+2 A(u) s_{h} .
\end{aligned}
$$

The initial data are

$$
\tilde{\iota}(0, x)=0, \quad \tilde{\iota}_{t}(0, x)=\tilde{h}_{x}(0)-A\left(u_{0}\right) \tilde{h}(0)=\left(h_{0, x}+h_{0, t x}\right)-A\left(u_{0}\right)\left(h_{0}+h_{0, t}\right) .
$$

As before, writing the Jin-Xin model we have

$$
\left\{\begin{array}{ccc}
\tilde{\iota}_{t}+p_{x} & = & -\int_{0}^{t} e^{-t}\left(D A(u)\left(u_{t} \times \tilde{h}-u_{x} \times \tilde{\iota}\right)+2 A(u) s_{h}\right) d \tau \\
p_{t}+\tilde{\iota}_{x} & = & A(u) \tilde{\iota}-p-2 s_{h}
\end{array}\right.
$$

and replacing $p$ with $\tilde{p}=p-e^{-t} \int_{-\infty}^{x}\left(A\left(u_{0}\right) \tilde{h}_{0}-h_{0, x}\right)$, defining $\tilde{\iota}=\iota^{-}+\iota^{+}, \tilde{p}=\iota^{+}-\iota^{-}$we obtain the kinetic scheme

$$
\left\{\begin{aligned}
\iota_{t}^{-}-\iota_{x}^{-} & =-\frac{I+A(u)}{2} \iota^{-}+\frac{I-A(u)}{2} \iota^{+}-s_{h}(t, x)+s_{\iota}(t, x) \\
\iota_{t}^{+}+\iota_{x}^{+} & =\frac{I+A^{(u)}}{2} \iota^{-}-\frac{I-A^{(u)}}{2} \iota^{+}+s_{h}(t, x)+s_{\iota}(t, x)
\end{aligned}\right.
$$

with initial data $\iota^{-}(0)=\iota^{+}(0)=0$ and source term

$$
\begin{aligned}
s_{\iota}(t, x)= & -\frac{e^{-t}}{2}\left(A\left(u_{0}\right) \tilde{h}_{0}-h_{0, x}\right)+\frac{1}{2} \int_{0}^{t} e^{\tau-t}\left(D A(u)\left(u_{t} \times \tilde{h}-u_{x} \times \tilde{\iota}\right)+A(u) 2 s_{h}\right) d \tau \\
\left\|s_{\iota}\right\|_{L^{1}} \leq & C\left(1+\sup _{\tau \in[0, t]}\left(\left\|\tilde{u}_{x}(\tau)\right\|_{L^{1}}+\left\|\tilde{u}_{t}(\tau)\right\|_{L^{1}}+\|\tilde{h}(\tau)\|_{L^{1}}+\|\tilde{\iota}(\tau)\|_{L^{1}}\right)\right) \delta_{1} e^{-t / 2} \\
& +C \sup _{\tau \in[0, t]}\left(\left(\left\|\tilde{u}_{x x}(\tau)\right\|_{L^{1}}+\left\|\tilde{u}_{t x}(\tau)\right\|_{L^{1}}\right)\left(\|\tilde{h}(\tau)\|_{L^{1}}+\|\tilde{\iota}(\tau)\|_{L^{1}}\right)\right), \\
\left\|s_{\iota, x}\right\|_{L^{1}} \leq & C\left(1+\sup _{\tau \in[0, t]}\left(\left\|\tilde{u}_{x x}(\tau)\right\|_{L^{1}}+\left\|\tilde{u}_{t, x}(\tau)\right\|_{L^{1}}+\left\|\tilde{h}_{x}(\tau)\right\|_{L^{1}}+\left\|\tilde{\iota}_{x}(\tau)\right\|_{L^{1}}\right)\right) \delta_{1} e^{-t / 2} \\
& +C \sup _{\tau \in[0, t]}\left(\left(\left\|\tilde{u}_{x x}(\tau)\right\|_{L^{1}}+\left\|\tilde{u}_{t x}(\tau)\right\|_{L^{1}}\right)\left(\left\|\tilde{h}_{x}(\tau)\right\|_{L^{1}}+\left\|\tilde{\iota}_{x}(\tau)\right\|_{L^{1}}\right)\right) .
\end{aligned}
$$

It is important to notice here that, as a consequence of the regularity estimates of the next section, all these equations have initial data in $L^{1}$ and source terms which have $L^{1}(\mathbb{R})$ norm of order $e^{-t} \delta_{1}+\delta_{0}^{3}$, at least in a time time interval $[0, T]$. The interaction terms we put in evidence in $s_{g}, s_{h}, s_{\iota}$ have to be studied carefully, because a priori we only know that their $L^{1}([0, T] \times \mathbb{R})$ norm is of order $T \delta_{0}^{3}$, i.e. arbitrarily large. Observe also that if the $x$ derivatives of $\tilde{u}_{x}, \tilde{u}_{t}, \tilde{h}, \tilde{\iota}$ is bounded in $L^{1}$ by $C \delta_{0}^{2}$, we can obtain similar $L^{1}$ estimates for $s_{g}, s_{h}, s_{\iota}$.

Notice that by construction $\tilde{\iota}=\iota^{+}+\iota^{-}=h^{-}-h^{+}$, which is the perturbation counter part of (2.33).

Remark 2.2. We want to put in evidence that with our decomposition of $u$ the function $u_{0, t}$ can be considered as an initial data for $\tilde{u}$.

As we said, this is important in the hyperbolic rescaling, because the correct rescaled initial data is $u_{0}+\epsilon u_{t}$. In particular, if $\epsilon u_{t} \notin L^{1}$, then in general for $\epsilon \rightarrow 0$ there is a jump at $t=0$. For example one can consider the simple model

$$
u_{t}=\epsilon\left(u_{x x}-u_{t t}\right) .
$$

with initial data $u(0)=0, u_{t}(0)=\epsilon^{-1}$. Then the solution is clearly $1-e^{-t / \epsilon}$, which converges to $u(t) \equiv 1$, $t>0$. Thus the hyperbolic limit should have the initial data $u(0)=1$.

In the next section we study regularity estimates on the solutions of these schemes assuming that the initial data and its derivatives have small $L^{1}$ norm. We prove uniform estimates on the solution on a small time interval. As a consequence the above schemes have solutions bounded in $L^{1}$ at least for small $t$. Note that this is a priori not clear for $\iota$, since in the definition (2.42) one may believe that $\iota$ is only in $L^{\infty}$. 
Remark 2.3. The introduction of the variables $\left(g^{-}, g^{+}\right),\left(\iota^{-}, \iota^{+}\right)$is due to the following two facts:

(1) for the equation (2.1) with $u \in \mathbb{R}$, the coordinates for which one has $L^{1}$ decay are the kinetic variables

$$
f^{-}=\frac{u_{x}+u_{t}}{2}, \quad f^{+}=\frac{u_{x}-u_{t}}{2} .
$$

Note hoverer that the initial data of the derivative of $(2.1)$ is $u_{x}, u_{t x}$. This means that we have to find some integrated variable for which we can write the kinetic model. For the perturbation $h, \iota$, this is particularly complicated;

(2) the wave structure of (2.1) needs to know the effective flux for $f^{-}, f^{+}$. This is why we introduce $g^{-}$, and $g^{+}$. A similar situation for $h$ leads to the introduction of $\iota$.

If the system were in conservation form, the variables $g^{ \pm}$have a clear interpretation as the time derivatives of $F^{ \pm}$.

Note moreover that if $g^{+}+g^{-}$is $\tilde{u}_{t}$, no clear meaning can be given to $\iota$. Also for $g$ we know that $g^{-}+g^{+}=f^{-}-f^{+}$, so the kinetic $g^{-}, g^{+}$separately do not have a clear meaning. Nevertheless, the functionals we introduce depends essentially on points 1),2) above.

\section{Existence AND REgularity OF THE SOLUTION}

Our aim here is to use the a priori assumption on smallness of the $L^{1}$ norm of $\left(f^{-}, f^{+}\right)$in some time interval $[0, T]$ to obtain estimates on $\left(f_{x}^{-}, f_{x}^{+}\right)$in $L^{1}$ and $L^{\infty}$. Similar estimates can be done for $\left(g^{-}, g^{+}\right)$, $\left(h^{-}, h^{+}\right)$and $\left(\iota^{-}, \iota^{+}\right)$.

When we consider an infinitesimal perturbation $h$ of (2.1) (i.e. the solution to (2.34)) and its effective flux $\iota$, as a general rule we can just replace

$$
u_{x} \longleftrightarrow h, \quad u_{t} \longleftrightarrow \iota
$$

a part from a source due to the non conservative form.

We show that the estimates derive from the following theorem:

Theorem 3.1. Consider the relaxation model

$$
\left\{\begin{array}{llc}
m_{t}+n_{x} & = & s_{1}(t, x) \\
n_{t}+m_{x} & = & A(t, x) m-n+s_{2}(t, x)
\end{array}\right.
$$

where $\|A-\bar{A}\|_{L^{1}},\left\|\partial_{x} A\right\|_{L^{1}} \leq C \delta_{0}$ and source terms $s_{1}, s_{2}$ satisfying

$$
\begin{gathered}
\int_{\mathbb{R}}\left(\left|s_{1}(t, x)\right|+\left|s_{2}(t, x)\right|\right) d x \leq C \delta_{1} e^{-t / 2}+C\left(\delta_{1} e^{-t / 2}+C \delta_{0}^{2}\right) \sup _{\tau \in[0, t]}\left(\|m(\tau)\|_{L^{1}}+\|n(\tau)\|_{L^{1}}\right), \\
(3.3) \int_{\mathbb{R}}\left(\left|s_{1, x}(t, x)\right|+\left|s_{2, x}(t, x)\right|\right) d x \leq C \delta_{1} e^{-t / 2}+C\left(\delta_{1} e^{-t / 2}+C \delta_{0}^{2}\right) \sup _{\tau \in[0, t]}\left(\left\|m_{x}(\tau)\right\|_{L^{1}}+\left\|n_{x}(\tau)\right\|_{L^{1}}\right) .
\end{gathered}
$$

Then if the initial data $m_{0}, n_{0}$ satisfy

$$
\int_{\mathbb{R}}\left|m_{0}(x)+n_{0}(x)\right|+\left|m_{0}(x)-n_{0}(x)\right| d x \leq 2 \delta_{0}, \quad \int_{\mathbb{R}}\left|m_{0, x}(x)\right|+\left|n_{0, x}(x)\right| d x \leq K,
$$

we have the estimates

$$
\int_{\mathbb{R}}|m(t, x)+n(t, x)|+|m(t, x)-n(t, x)| d x \leq 4 \delta_{0}, \quad \int_{\mathbb{R}}\left|m_{x}(t, x)\right|+\left|n_{x}(t, x)\right| d x \leq 32 \kappa C \delta_{0}^{2},
$$

for $t=\bar{t}=\left(C \delta_{0}\right)^{-2}$. The constant $\kappa<\infty$ depends only on the Green kernel for the linear problem (3.2) with $A(t, x)=\bar{A}$ and $s_{1}=s_{2}=0$.

First of all it is clear that we can repeat the estimate at any time interval $[t, t+\bar{t}]$, obtaining regularity estimates in $[\bar{t}, T+\bar{t}]$, once we prove that $m(t), n(t)$ satisfy (3.4) for all $t \in[0, T]$ (actually, due to (3.5) we need only the $L^{1}$ norm estimate and the regularity assumptions on the initial data). The $L^{1}$ norm we used in the above theorem ( $L^{1}$ norm of the kinetic variables) is clearly equivalent to the standard $L^{1}$ norm.

Second, the equation for $\tilde{u}_{x}$ are of the form (3.2), so that we obtain the $L^{1}$ norm estimates for $\tilde{u}_{t}, \tilde{u}_{x x}$, $\tilde{u}_{t x}$. The estimates on $\tilde{u}_{x}$ can be used to study the equation for $\tilde{u}_{t}$, because they appears in the source term $s_{g}, s_{g, x}$ (note that this justify the $C^{2}$ in $(3.3)$ ): this yields estimates on the $L^{1}$ norm of $\tilde{p}, \tilde{p}_{x}$. 
A similar analysis can be applied also to $\tilde{h}, \tilde{\iota}$, obtaining estimates for $\tilde{h}_{x}, \tilde{\iota}, \tilde{\iota}_{x}, \tilde{\iota}_{t x}$ (with $\tilde{\iota}$ used in $s_{\iota}$ ). In particular, using the second estimate of (3.5), it follows that we estimate $\tilde{u}_{t x}$ in $L^{1}$, hence we conclude $u_{t x} \in L^{1}$. Similarly, for the perturbation $h$, we have that $h_{t} \in L^{1}$.

In terms of the kinetic components $\left(f^{-}, f^{+}\right),\left(g^{-}, g^{+}\right),\left(g^{-}, g^{+}\right),\left(g^{-}, g^{+}\right)$, this means that if the initial data are in $L^{1}$, they remains in $L^{1}$ for small time and their $x$ derivatives are bounded. Thus the kinetic formulations of Section 2.1 have smooth solution with bounded $L^{1}$ norm, ate least in the small time interval $[0,7]$.

Remark 3.2. As we said before, while clearly $u_{x} \in L^{1}$ for small $t$, it is not (at least for the author) sufficiently clear that after all the transformations of Section 2.1 we obtain local in time $L^{1}$ solutions.

3.0.4. Proof of Theorem 3.1. For shortness define

$$
\|(m(t), n(t))\|_{L^{1}}=\int_{\mathbb{R}}|m(t, x)+n(t, x)|+|m(t, x)-n(t, x)| d x .
$$

Denote the left, right eigenvalues and eigenvectors of $\bar{A}$ by $\bar{l}_{i}, \bar{r}_{i}$ and $\bar{\lambda}_{i}$, respectively. The norm in $\mathbb{R}^{n}$ is $|v|=\sup _{i}\left|\left\langle\bar{l}_{i}, v\right\rangle\right|$.

The first intergal estimate follows easily by noticing that in kinetic variables

$$
m=\sum_{i}\left(m_{i}^{+}+m_{i}^{-}\right) \bar{r}_{i}, \quad n=\sum_{i}\left(m_{i}^{+}-m_{i}^{-}\right) \bar{r}_{i}
$$

we have the estimate

$$
\begin{aligned}
\frac{d}{d t}\left\|m_{i}^{-}(t)\right\|+\left\|m_{i}^{+}(t)\right\|_{L^{1}}= & \int_{\mathbb{R}} \frac{1+\lambda_{i}(t, x)}{2}\left(\operatorname{sgn}\left(z^{+}\right) z^{-}-\left|z^{-}\right|\right) d x \\
& +\frac{1-\lambda_{i}(t, x)}{2}\left(\operatorname{sgn}\left(z^{-}\right) z^{+}-\left|z^{+}\right|\right) d x \\
& +C \delta_{0} \sum_{i} \sup _{\tau \in[0, t]}\left(\left\|m_{i}^{-}(\tau)\right\|+\left\|m_{i}^{+}(\tau)\right\|_{L^{1}}\right)+C\left(e^{-t} \delta_{1}+\delta_{0}^{2}\right) \\
\leq & C \delta_{0} \sum_{i} \sup _{\tau \in[0, t]}\left(\left\|m_{i}^{-}(\tau)\right\|+\left\|m_{i}^{+}(\tau)\right\|_{L^{1}}\right)+C\left(e^{-t} \delta_{1}+\delta_{0}^{2}\right) .
\end{aligned}
$$

Integrating in $t$ and assuming $t \leq\left(4 C n \delta_{0}\right)^{-1}$, the result follows if $\delta_{1} \leq \delta_{0} /(4 C)$.

The proof of the second estimate (3.5) relies on estimates on the Green kernel $\Gamma(t, x)$ for the linear system

$$
\left\{\begin{array}{lll}
m_{t}+n_{x} & = & 0 \\
n_{t}+m_{x} & = & \bar{A} m-n
\end{array}\right.
$$

Since $\bar{A}=\sum_{i} \bar{\lambda}_{i}\left(\bar{l}_{i} \times \bar{r}_{i}\right)$, clearly

$$
\Gamma(t, x)=\sum_{i} \Gamma_{i}(t, x)\left(\bar{l}_{i} \times \bar{r}_{i}\right),
$$

where $\Gamma_{i}(t, x)$ are the Green kernels of the $2 \times 2$ system

$$
\left\{\begin{array}{llc}
m_{t}+n_{x} & = & 0 \\
n_{t}+m_{x} & = & \bar{\lambda}_{i} m-n
\end{array}\right.
$$


Using the results of $[7,18,23]$, we have that each $\Gamma_{i}$ satisfies the estimates

$$
\begin{aligned}
\partial_{x}^{k} \Gamma_{i}(t, x)= & \partial_{x}^{k}\left(\frac{1}{2 \sqrt{\left(1-\bar{\lambda}_{i}^{2}\right)(1+t)}} e^{-\left(x^{2}-\bar{\lambda}_{i} t\right)^{2} /\left(4\left(1-\bar{\lambda}_{i}^{2}\right)(1+t)\right)}\right)\left(\begin{array}{cc}
1 & 0 \\
0 & 0
\end{array}\right) \chi\{|x| \leq t\} \\
& \partial_{x}^{k+1}\left(\frac{1}{2 \sqrt{\left(1-\bar{\lambda}_{i}^{2}\right)(1+t)}} e^{-\left(x^{2}-\bar{\lambda}_{i} t\right)^{2} /\left(4\left(1-\bar{\lambda}_{i}^{2}\right)(1+t)\right)}\right)\left(\begin{array}{cc}
0 & 1 \\
-1 & 0
\end{array}\right) \chi\{|x| \leq t\} \\
& \partial_{x}^{k+2}\left(\frac{1}{2 \sqrt{\left(1-\bar{\lambda}_{i}^{2}\right)(1+t)}} e^{-\left(x^{2}-\bar{\lambda}_{i} t\right)^{2} /\left(4\left(1-\bar{\lambda}_{i}^{2}\right)(1+t)\right)}\right)\left(\begin{array}{cc}
0 & 0 \\
0 & 1
\end{array}\right) \chi\{|x| \leq t\} \\
& +e^{-\left(1+\bar{\lambda}_{i}\right) t / 2 \partial_{x}^{k} \delta(x+t)}\left(\begin{array}{c}
1 / 2 \\
-1 / 2 \\
-1 / 2 \\
1 / 2
\end{array}\right)+e^{-\left(1-\bar{\lambda}_{i}\right) t / 2} \partial_{x}^{k} \delta(x-t)\left(\begin{array}{cc}
1 / 2 & 1 / 2 \\
1 / 2 & 1 / 2
\end{array}\right) \\
& +\partial_{x}^{k}\left(e^{-\left(x^{2}-\bar{\lambda}_{i} t\right)^{2} /\left(4\left(1-\bar{\lambda}_{i}^{2}\right)(1+t)\right)}\right)\left(\begin{array}{cc}
\frac{\mathcal{O}(1)}{1+t} & \frac{\mathcal{O}(1)}{(1+t)^{3 / 2}} \\
\frac{\mathcal{O}(1)}{(1+t)^{3 / 2}} & \frac{\mathcal{O}(1)}{(1+t)^{2}}
\end{array}\right) \chi\{|x| \leq t\} .
\end{aligned}
$$

It follows that

$$
\begin{aligned}
\|\Gamma(t) *(u, v)\|_{L^{1}} & \leq\|(u, v)\|_{L^{1}} \leq\|u\|_{L^{1}}+\kappa \frac{\|v\|_{L^{1}}}{\sqrt{1+t}}+e^{-c t}\|(u, v)\|_{L^{1}}, \\
\left\|\partial_{x}(\Gamma(t) *(u, v))\right\|_{L^{1}} & \leq \kappa \frac{\|u\|_{L^{1}}}{\sqrt{1+t}}+\kappa \frac{\|v\|_{L^{1}}}{1+t}+e^{-c t}\left\|\left(u_{x}, v_{x}\right)\right\|_{L^{1}} \\
& =\kappa \frac{\|u\|_{L^{1}}}{\sqrt{1+t}}+\kappa \frac{\left\|v_{x}\right\|_{L^{1}}}{\sqrt{1+t}}+\kappa \frac{\|v\|_{L^{1}}}{(1+t)^{3 / 2}}+e^{-c t}\left\|\left(u_{x}, v_{x}\right)\right\|_{L^{1}},
\end{aligned}
$$

where we used also the conservation estimate (3.6), valid for the special system (3.8).

Write the solution to $(3.2)$ as

$$
\left(\begin{array}{c}
m \\
n
\end{array}\right)=\Gamma(t) *\left(\begin{array}{c}
m(0) \\
n(0)
\end{array}\right)+\int_{0}^{t} \Gamma(t-\tau) *\left(\begin{array}{c}
s_{1}(\tau) \\
(A(\tau))-\bar{A}) m(\tau)+s_{2}(\tau)
\end{array}\right) d \tau
$$

By using formula (3.10) and estimates (3.3) we obtain for $t=c / \delta_{0}, c$ small

$$
\begin{aligned}
\left\|\left(m_{x}(t), n_{x}(t)\right)\right\|_{L^{1}} \leq & \frac{\kappa}{\sqrt{1+t}}\|(m(0), n(0))\|_{L^{1}}+e^{-c t}\left\|\left(m_{x}(0), n_{x}(0)\right)\right\|_{L^{1}} \\
& +\kappa C \int_{0}^{t} \frac{1}{\sqrt{1+(t-\tau)}}\left(\delta_{0}\left\|m_{x}(\tau)\right\|_{L^{1}}+\delta_{0} \frac{\|m(\tau)\|_{L^{1}}}{1+(t-\tau)}\right) d \tau \\
& +\kappa C \int_{0}^{t} \frac{1}{\sqrt{1+(t-\tau)}}\left(\delta_{1} e^{-t / 2}+\left(\delta_{1} e^{-t / 2}+C \delta_{0}^{2}\right) \sup _{[0, \tau]}\left(\|m\|_{L^{1}}+\|n\|_{L^{1}}\right)\right) d \tau \\
& +C \int_{0}^{t} e^{\tau-t}\left(\delta_{0}\left\|m_{x}(\tau)\right\|_{L^{1}}+\delta_{1} e^{-t / 2}+\left(\delta_{1} e^{-t / 2}+C \delta_{0}^{2}\right) \sup _{[0, \tau]}\left(\left\|m_{x}\right\|_{L^{1}}+\left\|n_{x}\right\|_{L^{1}}\right)\right) d s \\
\leq & \frac{4 \kappa\left(\delta_{0}+C \delta_{1}\right)}{\sqrt{1+t}}+\left(C \delta_{1}+K\right) e^{-c t}+2 \kappa C \delta_{0} \int_{0}^{t} \frac{\|\left(m_{x}(\tau), n_{x}(\tau) \|_{L^{1}}\right.}{\sqrt{1+(t-\tau)}} d \tau \\
& +8 \kappa C \delta_{0}^{2}+4 \kappa C^{2} \delta_{0}^{3} \sqrt{1+t} .
\end{aligned}
$$

We used the assumption $\delta_{0}$ sufficiently small.

Set now $\delta_{1} \leq \delta_{0} / C$. By Gronwall estimate it follows that we have the bound

$$
\left\|\left(m_{x}(t), n_{x}(t)\right)\right\|_{L^{1}} \leq \frac{31 \kappa C \delta_{0}}{\sqrt{1+t}}+4 K e^{-t}, \quad t \in\left[0,\left(C \delta_{0}\right)^{-2}\right] .
$$

In particular we see that if $\delta_{0}$ is sufficiently small (so that $4 K e^{-\bar{t}} \leq \kappa C \delta_{0}^{2}$ ), we recover (3.5).

In the following we will thus consider the various kinetic schemes with $L^{1}$ data and a priori assumption that the $L^{1}$ norm of the solution is bounded by $2 \delta_{0}$ in the time interval $[0, T]$. As we saw, this is correct, since small $L^{1}$ initial data give rise to small local in times $L^{1}$ solution. 
3.1. Lipschitz dependence w.r.t. $t$. The analysis on the linear Green kernel yields the Lipschitz dependence w.r.t. time. In fact we can write

$$
\tilde{u}_{t}+A(0) \tilde{u}_{x}=\tilde{u}_{x x}-\tilde{u}_{t t}+(A(0)-A(u)) \tilde{u}_{x}+e^{-t}\left(A(u) u_{0, t x}-u_{0, t x x}\right),
$$

so that we obtain the relaxation system

$$
\left\{\begin{array}{ccc}
\tilde{u}_{t}+\tilde{v}_{x} & = & \int_{0}^{t} e^{\tau-t}\left((A(u)-A(0)) \tilde{u}_{x}+e^{-\tau}\left(A(u) u_{0, t x}-u_{0, t x x}\right)\right) d \tau \\
\tilde{v}_{t}+\tilde{u}_{x} & = & A(0) \tilde{u}-\tilde{v}
\end{array}\right.
$$

with

$$
\tilde{v}(t, x)=\int_{0}^{t} \int_{-\infty}^{x} e^{\tau-t}\left((A(u)-A(0)) \tilde{u}_{x}+e^{-\tau}\left(A(u) u_{0, t x}-u_{0, t x x}\right)\right) d \tau-\int_{-\infty}^{x} \tilde{u}_{t}(t, y) d y \in L^{\infty}(\mathbb{R}) .
$$

We thus have by Duhamel formula

$$
\begin{aligned}
\left(\begin{array}{c}
\tilde{u}(t) \\
\tilde{v}(t)
\end{array}\right)= & \Gamma(t-s) *\left(\begin{array}{c}
\tilde{u}(s) \\
\tilde{v}(s)
\end{array}\right) \\
& +\int_{0}^{t} \Gamma(t-\tau)\left(\begin{array}{c}
\int_{0}^{\tau} e^{\varsigma-\tau}\left((A(u)-A(0)) \tilde{u}_{x}+e^{-\varsigma}\left(A(u) u_{0, t x}-u_{0, t x x}\right)\right) d \varsigma \\
0
\end{array}\right) d \tau .
\end{aligned}
$$

The last integral is of order $C \delta_{0}^{2}(t-s)+C \delta_{1} e^{-t}$ in $L^{1}$, since $\left\||u| \tilde{u}_{x}\right\|_{L^{1}} \leq C \delta_{0}^{2}$ and (2.9), and a simple computation show that

$$
\left\|\left(\begin{array}{c}
\tilde{u}(s) \\
\tilde{v}(s)
\end{array}\right)-\Gamma(t-s) *\left(\begin{array}{c}
\tilde{u}(s) \\
\tilde{v}(s)
\end{array}\right)\right\|_{L^{1}} \leq C(t-s), \quad s \leq t,
$$

if the derivatives $\tilde{u}_{x}, \tilde{v}_{x}$ are bounded in $L^{\infty}$ by some constant.

Thus the assumption on boundedness of the $L^{1}$ norm of $\left(\tilde{u}_{x}, \tilde{v}_{x}\right)$ and the regularity estimates yield the $L^{1}$ Lipschitz dependence w.r.t. time. A similar Lipschitz dependence w.r.t. $t$ holds for the $x$ derivatives $\tilde{u}_{x}, \tilde{v}_{x}$, hence for $\tilde{u}_{t}$. A simple computation shows that the same Lipschitz dependence is thus valid for $u, u_{t}$

$$
\|u(t)-u(s)\|_{L^{1}}+\left\|u_{t}(t)-u_{t}(s)\right\|_{L^{1}} \leq C|t-s|
$$

3.2. Remarks about the structure of the Green kernel. If we consider a general linear relaxation system

$$
\left\{\begin{array}{l}
u_{t}+A_{11} u_{x}+A_{12} v_{x}=0 \\
v_{t}+A_{21} u_{x}+A_{22} v_{x}=-B v
\end{array}\right.
$$

with $u \in \mathbb{R}^{n}, v \in \mathbb{R}^{k}, B: \mathbb{R}^{k} \mapsto \mathbb{R}^{k}$ positive definite and

$$
A=\left[\begin{array}{ll}
A_{11} & A_{12} \\
A_{21} & A_{22}
\end{array}\right],
$$

symmetric with no eigenvectors in $\left(\mathbb{R}^{n}, 0\right)$ (Kawashima condition), then in [7] it is proved that the Green function $\Gamma$ has the special structure

$$
\Gamma(t, x)=\left[\begin{array}{cc}
\Gamma_{11}(t, x) & \partial_{x} \Gamma_{12}(t, x) \\
\partial_{x} \Gamma_{21}(t, x) & \partial_{x}^{2} \Gamma_{22}(t, x)
\end{array}\right]+\text { exponentially decaying terms }+ \text { higher order terms, }
$$

where all $\Gamma_{i j}$ behave like the heat kernel $G$.

Following the approach used to recover $L^{1}$ initial data for $f^{ \pm}$, we can prove that the part of the Green kernel $\Gamma$ corresponding to the initial data in $v$ are actually a derivative of some functions. This estimate is a refinement of (3.15), because it says that also some higher order term is a derivative.

Since in this paper we only consider the Jin-Xin relaxation model, we write this new form of the kernel for the $2 \times 2$ Jin-Xin model with average speed $\lambda$,

$$
\left\{\begin{array}{l}
u_{t}+v_{x}=0 \\
v_{t}+u_{x}=\lambda u-v
\end{array}\right.
$$


We recall that we have the estimates

$$
\begin{aligned}
\Gamma_{11}(t, x)= & \frac{1}{2 \sqrt{\left(1-\lambda^{2}\right)(1+t)}} e^{-\left(x^{2}-\lambda t\right)^{2} /\left(4\left(1-\lambda^{2}\right)(1+t)\right)} \\
& +\frac{1}{2}\left(e^{-(1+\lambda) t / 2} \delta(x+t)+e^{-(1-\lambda) t / 2} \delta(x-t)\right) \\
& +\frac{\mathcal{O}(1)}{1+t} e^{-\left(x^{2}-\lambda t\right)^{2} /\left(4\left(1-\lambda^{2}\right)(1+t)\right)}, \\
\Gamma_{12}(t, x)=- & \frac{\partial}{\partial x}\left(\frac{1}{2 \sqrt{\left(1-\lambda^{2}\right)(1+t)}} e^{-\left(x^{2}-\lambda t\right)^{2} /\left(4\left(1-\lambda^{2}\right)(1+t)\right)}\right) \\
+ & \frac{1}{2}\left(-e^{-(1+\lambda) t / 2} \delta(x+t)+e^{-(1-\lambda) t / 2} \delta(x-t)\right) \\
+ & \frac{\mathcal{O}(1)}{(1+t)^{3 / 2}} e^{-\left(x^{2}-\lambda t\right)^{2} /\left(4\left(1-\lambda^{2}\right)(1+t)\right)} .
\end{aligned}
$$

This is how the initial data $(\delta(x), 0)$ evolves in time in the coordinates $(u, v)$.

For the initial data $(0, H(x))$, where $H$ is the Heaviside function, then the substitution $v=\tilde{v}+e^{-t} H(x)$ transforms the Jin-Xin system into

$$
\left\{\begin{array}{l}
u_{t}+\tilde{v}_{x}=-e^{-t} \delta(x) \\
\tilde{v}_{t}+u_{x}=\lambda u-\tilde{v}
\end{array}\right.
$$

whose solution can be written as

$$
\left(\begin{array}{c}
u(t, x) \\
v(t, x)-e^{-t} H(t-x)
\end{array}\right)=-\int_{0}^{t}\left(\begin{array}{c}
\Gamma_{11}(t-s, x) \\
\Gamma_{12}(t-s, x)
\end{array}\right) e^{-s} d s=\left(\begin{array}{c}
\tilde{\Gamma}_{12}(t, x) \\
\tilde{\Gamma}_{22}-e^{-t} H(x-\lambda t)
\end{array}\right)
$$

By studying the heat equation with source

$$
u_{t}+\lambda u_{x}-\left(1-\lambda^{2}\right) u_{x x}=-e^{-t} \delta(x),
$$

one sees that the principal part is again a heat kernel travelling with speed $\lambda$, i.e.

$$
\begin{aligned}
\tilde{\Gamma}_{12}= & -\frac{1}{2 \sqrt{\left(1-\lambda^{2}\right)(1+t)}} e^{-\left(x^{2}-\lambda t\right)^{2} /\left(4\left(1-\lambda^{2}\right)(1+t)\right)} \\
& -\frac{1}{2}\left(e^{-(1-\lambda)|x| / 2-(t-|x|)} \chi_{[-t, 0]}(x)+e^{-(1+\lambda)|x| / 2-(t-|x|)} \chi_{[0, t]}(x)\right) \\
& + \text { higher order terms, } \\
\tilde{\Gamma}_{12}= & -\frac{\partial}{\partial x}\left(\frac{1}{2 \sqrt{\left(1-\lambda^{2}\right)(1+t)}} e^{-\left(x^{2}-\lambda t\right)^{2} /\left(4\left(1-\lambda^{2}\right)(1+t)\right)}\right) \\
& -\frac{1}{2}\left(e^{-(1-\lambda)|x| / 2-(t-|x|)} \chi_{[-t, 0]}(x)+e^{-(1+\lambda)|x| / 2-(t-|x|)} \chi_{[0, t]}(x)\right) \\
& -e^{-t} H(x)+\text { higher order terms. }
\end{aligned}
$$

\section{Center manifold of travelling profiles for Relaxation}

In this section we extend the decomposition in travelling profile used in [3], [5], to the Jin-Xin relaxation model. The fundamental feature of this relaxation system is that, due to the linear stability condition $(2.3)$, it is possible to identify an exact travelling wave $\phi$ by means of only one kinetic component $f^{-}$or $f^{+}$. More precisely, if the solution $u$ is an exact travelling profile $\phi_{i}(x-\sigma t)$ of the $i$-th passing through $u$ with speed $\sigma$, then to identify exactly the profile it suffices to know either the $i$-th component $f_{i}^{-}$of $f^{-}$or the $i$-th component $f_{i}^{+}$of $f^{+}$.

For a real solution, this suggests that by looking at different scalar components $f_{i}^{-}, i=1, \ldots, n$, of the vector valued function $f^{-}$we obtain $n$ different travelling profiles $\phi_{i}^{-}, i=1, \ldots, n$. Similarly, by looking to the scalar components $f_{i}^{+}, i=1 \ldots, n$, of the function $f^{+}$, one obtain $n$ different travelling profiles $\phi_{i}^{+}, i=1, \ldots, n$. It is thus natural to expect a decomposition into $2 n$ travelling waves, each one interacting with the other waves. 
We divide this section into two parts: in the first part we show the existence of a center manifold of travelling profiles for each family $i=1, \ldots, n$, in the second one we write the center manifold in terms of the kinetic components $f^{-}$and $f^{+}$. For our Jin-Xin model this is particularly simple, but it is a of fundamental importance when proving BV bounds.

The decomposition of $u$ (or $h$ ) into $2 n$ travelling profiles is done in the next section.

4.1. Center manifold of travelling profiles. This part is the relaxation analog of the analysis of the center manifold of travelling profiles done in [5].

We consider the equation for a travelling profile of speed $\sigma$, i.e. $u_{t}+\sigma u_{x}=0$ and $\sigma_{x}=\sigma_{t}=0$ : equation (2.1) becomes the ODE

$$
(A(u)-\sigma I) u_{x}=\left(1-\sigma^{2}\right) u_{x x} .
$$

We can write (4.1) as a first order system

$$
\left\{\begin{array}{ccc}
u_{x} & = & p \\
\left(1-\sigma^{2}\right) p_{x} & = & (A(u)-\sigma I) p \\
\sigma_{x} & = & 0
\end{array}\right.
$$

Linearizing the system in the equilibrium $\left(0,0, \lambda_{i}(0)\right)$, we obtain that the matrix

$$
\left[\begin{array}{ccc}
0 & I & 0 \\
0 & \left(A(0)-\lambda_{i}(0) I\right) /\left(1-\lambda_{i}(0)^{2}\right) & 0 \\
0 & 0 & 0
\end{array}\right]
$$

has a (generalized) null space of dimension $n+2$, namely

$$
N_{i}=\left\{\left(u, p=s r_{i}(0), \sigma\right), u \in \mathbb{R}^{n}, s, \sigma \in \mathbb{R}\right\},
$$

Thus there exists a center manifold of dimension $n+2$, defined in a neighborhood of 0 of radius $5 \delta_{2}$, which can be written by

$$
p_{j}=\phi_{j i}\left(u, p_{i}, \sigma\right)
$$

where $p_{j}=\left\langle l_{j}, p\right\rangle$ is the $j$ component of the vector $p$, and $\phi_{j i}$ are smooth function. Since for $p=0$ we are on the equilibria $(u, 0, \sigma)$, and these equilibria must belong to the center manifold, it follows that

$$
p_{i}=0 \quad \Longrightarrow \quad \phi_{j i}(u, 0, \sigma)=0,
$$

so that we can write $\phi_{j i}\left(u, p_{i}, \sigma\right)=p_{i} \tilde{\phi}_{j i}\left(u, p_{i}, \sigma\right)$ for some new smooth function $\tilde{\phi}_{j i}$. Using the fact that the center manifold is tangent to $N_{i}$, so that $\tilde{\phi}_{j i}\left(0,0, \lambda_{i}(0)\right)=\delta_{j i}$, one can show that the map

$$
p_{i} \mapsto v_{i} \doteq p_{i}\left|r_{i}(0)+\sum_{j \neq i} \tilde{\phi}_{j i} r_{j}(0)\right|
$$

is invertible in the neighborhood of $\left(0,0, \lambda_{i}(0)\right)$. Thus we parameterize the center manifold by $\left(u, v_{i}, \sigma\right)$, and we can write

$$
p=v_{i} \tilde{r}_{i}\left(u, v_{i}, \sigma\right), \quad\left|\tilde{r}_{i}(u)\right|=1,
$$

for some vector function $\tilde{r}_{i}$. We call the function $\tilde{r}_{i}$ the generalized eigenvector.

This happens for any family $i, i=1, \ldots, n$, so that we have $n$ generalized eigenvectors $\tilde{r}_{j}\left(u, v_{j}, \sigma_{j}\right)$, $j=1, \ldots, n$, defined in a neighborhood of radius $5 \delta_{2}$ of $\left(0,0, \lambda_{i}(0)\right)$, with $\delta_{2}$ sufficiently small. We can assume also that $\left|\lambda_{i}(0)\right|+5 \delta_{2}<1-c$. Since $\delta_{2}$ is a constant which depends only on the non linearity $A(u)$, we can assume also that $\delta_{0} \ll \delta_{2}$.

We can now find the reduced ODE on the center manifolds, and the relations among the derivatives of $\tilde{r}_{i}$. Subsituting (4.3) into (4.2) one obtains

$$
\left\{\begin{array}{ccc}
u_{x} & & v_{i} \tilde{r}_{i} \\
\left(1-\sigma^{2}\right)\left(v_{i, x} \tilde{r}_{i}+v_{i} r_{i, x}\right) & = & (A(u)-\sigma I) v_{i} \tilde{r}_{i} \\
\sigma_{x} & = & 0
\end{array}\right.
$$


and taking the scalar product of the second equation with $\tilde{r}_{i}$ (by the second of (4.3) it follows $\left\langle r_{i}, r_{i, x}\right\rangle=0$ ), we obtain the reduced system

$$
\left\{\begin{array}{ccc}
u_{x} & = & v_{i} \tilde{r}_{i} \\
\left(1-\sigma^{2}\right) v_{i, x} & = & \left(\tilde{\lambda}_{i}(u, v, \sigma)-\sigma\right) v_{i} \\
\sigma_{x} & = & 0
\end{array}\right.
$$

where we define the generalized eigenvalue $\tilde{\lambda}_{i}$ as

$$
\tilde{\lambda}_{i}\left(u, v_{i}, \sigma\right) \doteq\left\langle r_{i}\left(u, v_{i}, \sigma\right), A(u) \tilde{r}_{i}\left(u, v_{i}, \sigma\right)\right\rangle .
$$

Multiplying the above equation for $\tilde{r}_{i}$ and subtracting it to the second equation in (4.4) we conclude that

$$
\left(1-\sigma^{2}\right) v_{i} D \tilde{r}_{i} \tilde{r}_{i}\left(u, v_{i}, \sigma\right)+\left(\tilde{\lambda}_{i}\left(u, v_{i}, \sigma\right)-\sigma\right) v_{i} \tilde{r}_{i, v}\left(u, v_{i}, \sigma\right)=\left(A(u)-\tilde{\lambda}_{i}\left(u, v_{i}, \sigma\right) I\right) \tilde{r}_{i}\left(u, v_{i}, \sigma\right) .
$$

As a consequence we obtain that when $v_{i}=0, \tilde{r}_{i}(u, 0, \sigma)=r_{i}(u)$, i.e. we have the estimates

$$
\tilde{r}_{i}\left(u, v_{i}, \sigma\right)=r_{i}(u)+\mathcal{O}(1) v_{i}, \quad \tilde{\lambda}_{i}\left(u, v_{i}, \sigma\right)=\lambda_{i}(u)+\mathcal{O}(1) v_{i},
$$

where in the last equation we used the fact that the vectors $r_{i}, \tilde{r}_{i}$ have length 1.

4.2. Tangent vectors for travelling profiles in kinetic variables. We now show how it is possible to use the kinetic components $f^{-}, f^{+}$to parameterize the center manifold. We construct in fact new tangent vectors $\tilde{r}_{i}^{-}, \tilde{r}_{i}^{+}$which depend on $f^{-}, f^{+}$, respectively, instead of $u_{x}$. On a travelling profile with speed $\sigma$ one has

$$
u_{t}+\sigma u_{x}=0 \quad \Longrightarrow \quad(1-\sigma) f^{+}=(1+\sigma) f^{-}
$$

It follows that

$$
u_{x}=\frac{1}{2}\left(f^{+}+f^{-}\right)=\frac{1}{1-\sigma} f^{-}=\frac{1}{1+\sigma} f^{+} .
$$

Since on the center manifold is given by $u_{x}=v_{i} \tilde{r}_{i}$, we obtain that

$$
\begin{aligned}
& f^{-}=(1-\sigma) v_{i} \tilde{r}_{i}\left(u, v_{i}, \sigma\right)=f_{i}^{-} \tilde{r}_{i}\left(u, f_{i}^{-} /(1-\sigma), \sigma\right)=f_{i}^{-} \tilde{r}_{i}^{-}\left(u, f_{i}^{-}, \sigma\right), \\
& f^{+}=(1+\sigma) v_{i} \tilde{r}_{i}\left(u, v_{i}, \sigma\right)=f_{i}^{+} \tilde{r}_{i}\left(u, f_{i}^{+} /(1+\sigma), \sigma\right)=f_{i}^{+} \tilde{r}_{i}^{+}\left(u, f_{i}^{+}, \sigma\right) .
\end{aligned}
$$

where we define $f_{i}^{ \pm}=\left\langle\tilde{r}_{i}, f^{ \pm}\right\rangle$, i.e. $v_{i}=f_{i}^{ \pm} /(1 \pm \sigma)$. Thus the vectors $\tilde{r}_{i}^{ \pm}$are defined by the formulas

$$
\tilde{r}_{i}^{-}\left(u, f_{i}^{-}, \sigma\right) \doteq \tilde{r}_{i}\left(u, f_{i}^{-} /(1-\sigma), \sigma\right), \quad \tilde{r}_{i}^{+}\left(u, f_{i}^{+}, \sigma\right) \doteq \tilde{r}_{i}\left(u, f_{i}^{+} /(1+\sigma), \sigma\right) .
$$

We can thus rewrite the formula (4.7) for these new generalized eigenvectors, obtaining

$$
\tilde{r}_{i, f}^{ \pm}\left(u, f_{i}^{ \pm}, \sigma\right)=\frac{1}{1 \pm \sigma} \tilde{r}_{i, v}\left(u, f_{i}^{ \pm} /(1 \pm \sigma), \sigma\right)
$$

and

$$
\begin{aligned}
& (1+\sigma) f_{i}^{-} D \tilde{r}_{i}^{-} \tilde{r}_{i}^{-}\left(u, f_{i}^{-}, \sigma\right)+\left(\tilde{\lambda}_{i}^{-}-\sigma\right) f_{i}^{-} \tilde{r}_{i, f}^{-}\left(u, f_{i}^{-}, \sigma\right)=\left(A(u)-\tilde{\lambda}_{i}^{-} I\right) \tilde{r}_{i}^{-}\left(u, f_{i}^{-}, \sigma\right) \\
& (1-\sigma) f_{i}^{+} D \tilde{r}_{i}^{+} \tilde{r}_{i}^{+}\left(u, f_{i}^{+}, \sigma\right)+\left(\tilde{\lambda}_{i}^{+}-\sigma\right) f_{i}^{+} \tilde{r}_{i, f}^{+}\left(u, f_{i}^{+}, \sigma\right)=\left(A(u)-\tilde{\lambda}_{i}^{+} I\right) \tilde{r}_{i}^{+}\left(u, f_{i}^{+}, \sigma\right) .
\end{aligned}
$$

Note that also for $\tilde{r}_{i}^{ \pm}$the estimate (4.8) holds:

$$
\tilde{r}_{i, \sigma}^{ \pm}\left(u, f_{i}^{ \pm}, \sigma\right)=\mp \frac{f_{i}^{ \pm}}{(1 \pm \sigma)^{2}} \tilde{r}_{i, f}\left(u, f_{i}^{ \pm} /(1 \pm \sigma), \sigma\right)+\tilde{r}_{i, \sigma}\left(u, f_{i}^{ \pm} /(1 \pm \sigma), \sigma\right)=\mathcal{O}(1) f_{i}^{ \pm} .
$$

Finally, we can recover $f^{+}$from $f_{i}^{-}$or $f^{-}$from $f_{i}^{+}$by just using (4.9),

$$
f^{+}=\frac{1+\sigma}{1-\sigma} f_{i}^{-} \tilde{r}_{i}^{-}\left(u, f_{i}^{-}, \sigma\right), \quad f^{-}=\frac{1-\sigma}{1+\sigma} f_{i}^{+} \tilde{r}_{i}^{+}\left(u, f_{i}^{+}, \sigma\right) .
$$

Remark 4.1. We observe here that the vectors $f^{-}, f^{+}$are parallel if $u$ is a travelling profile. This is a special feature of the Jin-Xin scheme considered in this paper, which simplifies the computations: it does not hold for general BGK models.

Moreover, since the non linearity $A(u)$ depends only on the macroscopic state $u$, then it is natural to decompose $f^{-}, f^{+}$in travelling profiles passing through $u$. 


\section{DeCOMposition AND SOURCE TERMS}

Aim of this section is to decompose the couple of $2 n$ dimensional variables $\left(f^{-}, f^{+}\right),\left(g^{-}, g^{+}\right)$and $\left(h^{-}, h^{+}\right),\left(\iota^{-}, \iota^{+}\right)$as the sum of $2 n$ travelling profiles. The decomposition for the perturbations $h, \iota$ is the natural extension of the decomposition for $f, g$.

Let us explain what we are going to do. We consider each couple $\left(f^{-}, g^{-}\right),\left(f^{+}, g^{+}\right)$as the sum of $n$ travelling profile, passing through the same point $u$ but with a relative strength $f_{i}^{-}, f_{i}^{+}$and a speed $\sigma_{i}^{-}$, $\sigma_{i}^{+}$different for each family + and - . We say relative strength because the real strength of the wave is $u_{i, x}^{-}=f_{i}^{-} /\left(1-\sigma_{i}^{-}\right), u_{i, x}^{+}=f_{i}^{+} /\left(1+\sigma_{i}^{+}\right)$. The speed of each family is naturally assumed to be $g_{i}^{-} / f_{i}^{-}$, $g_{i}^{+} / f_{i}^{+}$.

In some sense, each kinetic variable has a decomposition which is independent on the other. However, when the speeds are the same and there is only the $\bar{i}$-th component for $f, g$ (i.e. only the components $f_{\bar{i}}^{-}$, $f_{\bar{i}}^{+}$are different from 0 ), and the speeds are the same, i.e. $\sigma_{\bar{i}}^{-}=\sigma_{\bar{i}}^{+}$, then we are on the center manifold. This shows that the decomposition identify exact travelling profiles, and that we actually replace the condition $\sigma_{x}=\sigma_{t}=0$ with $\sigma_{\bar{i}}^{-}=\sigma_{\bar{i}}^{+}$.

5.1. Decomposition in travelling profiles. We define

$$
\begin{aligned}
& \left\{\begin{array}{rlrl}
f^{-} & = & \sum_{i} f_{i}^{-} \tilde{r}_{i}^{-}\left(u, f_{i}^{-}, \sigma_{i}^{-}\right) & \sigma_{i}^{-}=\lambda_{i}(0)+\theta\left(-\frac{g_{i}^{-}}{f_{i}^{-}}\right), \\
g^{-} & = & \sum_{i}\left(g_{i}^{-}-\lambda_{i}(0) f_{i}^{-}\right) \tilde{r}_{i}^{-}\left(u, f_{i}^{-}, \sigma_{i}^{-}\right)
\end{array}\right. \\
& \left\{\begin{array}{lll}
f^{+} & = & \sum_{i} f_{i}^{+} \tilde{r}_{i}^{+}\left(u, f_{i}^{+}, \sigma_{i}^{+}\right) \\
g^{+} & = & \sum_{i}\left(g_{i}^{+}-\lambda_{i}(0) f_{i}^{+}\right) \tilde{r}_{i}^{+}\left(u, f_{i}^{+}, \sigma_{i}^{+}\right)
\end{array}\right.
\end{aligned}
$$

and similarly for $h$

$$
\left\{\begin{array}{ccc}
h^{ \pm} & = & \sum_{i} h_{i}^{ \pm} \tilde{r}_{i}^{ \pm}\left(u, f_{i}^{ \pm}, \sigma_{i}^{ \pm}\right) \\
\iota^{ \pm} & = & \sum_{i}\left(\iota_{i}^{ \pm}-\lambda_{i}(0) h_{i}^{ \pm}\right) \tilde{r}_{i}^{ \pm}\left(u, f_{i}^{ \pm}, \sigma_{i}^{ \pm}\right)
\end{array}\right.
$$

with $\sigma_{i}^{ \pm}$defined in $(5.1),(5.2)$.

The functions $\theta$ is a cutoff functions,

$$
\theta(x)=\left\{\begin{array}{lll}
x & |x| \leq 2 \delta_{2} \\
\text { smooth connection } & 2 \delta_{2}<|x|<3 \delta_{2} & |\theta| \leq 3 \delta_{2} . \\
0 & |x| \geq 3 \delta_{2}
\end{array}\right.
$$

Note that the center manifold is defined in a neighborhood of radius $5 \delta_{2}$, so that $\tilde{r}_{i}^{ \pm}\left(u, f_{i}^{ \pm}, \sigma_{i}^{ \pm}\right)$is meaningful for all values of $f, g$. Observe moreover that as a consequence of (4.13) the generalized eigenvectors $\tilde{r}_{i}^{ \pm}\left(u, f_{i}^{ \pm}, \sigma_{i}^{ \pm}\right)$are at least Lipschitz continuous.

Using the implicit mapping theorem as in [5], one can show that the maps (5.1), (5.2), (5.3) are invertible in a neighborhood of $(0,0)$. By regularity estimates we have that in a time interval $[0, T]$ the functions $f^{ \pm}, g^{ \pm}, h^{ \pm}, \iota^{ \pm}$are bounded in $L^{\infty}$ by $\mathcal{O}(1) \delta_{0}^{2}$. By choosing $\delta_{0}$ sufficiently small, we can thus assume that the decomposition in travelling profiles is well defined in $[0, T] \times \mathbb{R}$.

For shortness, we will use the notation

$$
\tilde{r}_{i}^{ \pm}=\tilde{r}_{i}^{ \pm}\left(u, f_{i}^{ \pm}, \sigma_{i}^{ \pm}\right), \quad \theta\left(-g_{i}^{ \pm} / f_{i}^{ \pm}\right)=\theta_{i}^{ \pm} .
$$

Since the variables $u_{x}, f, g$ and $h, \iota$ are not completely independent, we obtain some relations among the $f_{i}^{ \pm}, g_{i}^{ \pm}$and $h_{i}^{ \pm}, \iota_{i}^{ \pm}$. The most easy ones are of course

$$
\tilde{u}_{x}=f^{+}+f^{-}=\sum_{j}\left(f_{j}^{+} \tilde{r}_{j}^{+}+f_{j}^{-} \tilde{r}_{j}^{-}\right), \quad \tilde{h}=h^{+}+h^{-}=\sum_{j}\left(h_{j}^{+} \tilde{r}_{j}^{+}+h_{j}^{-} \tilde{r}_{j}^{-}\right)
$$

and from (2.33)

$$
\tilde{u}_{t}=u_{t}-e^{-t} u_{t}(0)=\sum_{j}\left(g_{j}^{-}-\lambda_{j}(0) f_{j}^{-}\right) \tilde{r}_{j}^{-}+\sum_{j}\left(g_{j}^{+}-\lambda_{j}(0) f_{j}^{+}\right) \tilde{r}_{j}^{+}=\sum_{j}\left(f_{j}^{-} \tilde{r}_{j}^{-}-f_{j}^{+} \tilde{r}_{j}^{+}\right),
$$

and similarly for $\tilde{\iota}$,

$$
\tilde{\iota}=\sum_{j}\left(\iota_{j}^{-}-\lambda_{j}(0) h_{j}^{-}\right) \tilde{r}_{j}^{-}+\sum_{j}\left(\iota_{j}^{+}-\lambda_{j}(0) h_{j}^{+}\right) \tilde{r}_{j}^{+}=\sum_{j}\left(h_{j}^{-} \tilde{r}_{j}^{-}-h_{j}^{+} \tilde{r}_{j}^{+}\right) .
$$


One estimate which will be used in the following is the difference among $\tilde{r}_{i}^{+}-\tilde{r}_{i}^{-}$:

$$
\begin{aligned}
\tilde{r}^{+}-\tilde{r}^{-}= & \mathcal{O}(1)\left(\frac{f_{i}^{-}}{1-\sigma_{i}^{+}}-\frac{f_{i}^{+}}{1+\sigma_{i}^{-}}\right)+\mathcal{O}(1)\left(\left|f_{i}^{-}\right|+\left|f_{i}^{+}\right|\right)\left(\sigma_{i}^{+}-\sigma_{i}^{-}\right) \\
= & \mathcal{O}(1)\left(f_{i}^{-}-f_{i}^{+}+\sigma_{i}^{+} f_{i}^{+}+\sigma_{i}^{-} f_{i}^{-}\right)+\mathcal{O}(1)\left(\left|f_{i}^{-}\right|+\left|f_{i}^{+}\right|\right)\left(\sigma_{i}^{+}-\sigma_{i}^{-}\right) \\
= & \mathcal{O}(1)\left(g_{i}^{-}+g_{i}^{+}-\left(1+\lambda_{i}\right) f_{i}^{-}+\left(1-\lambda_{i}\right) f_{i}^{+}\right)+\mathcal{O}(1)\left(g_{i}^{-}+\theta_{i}^{-} f_{i}^{-}\right) \\
& +\mathcal{O}(1)\left(g_{i}^{+}+\theta_{i}^{+} f_{i}^{+}\right)+\mathcal{O}(1)\left(\left|f_{i}^{-}\right|+\left|f_{i}^{+}\right|\right)\left(\sigma_{i}^{+}-\sigma_{i}^{-}\right) .
\end{aligned}
$$

By using (5.6) in (5.5) we obtain the relation among the $g_{i}^{ \pm}$and the $f_{i}^{ \pm}$:

$$
\begin{aligned}
0= & \sum_{i}\left(g_{i}^{-}-\lambda_{i}(0) f_{i}^{-}\right) \tilde{r}_{i}^{-}+\left(g_{i}^{+}-\lambda_{i}(0) f_{i}^{+}\right) \tilde{r}_{i}^{+}-\sum_{i} f^{-} \tilde{r}_{i}^{-}-f_{i}^{+} \tilde{r}_{i}^{+} \\
= & \sum_{i}\left(g_{i}^{-}-\left(1+\lambda_{i}(0)\right) f_{i}^{-}\right) \tilde{r}_{i}^{-}+\sum_{i}\left(g_{i}^{+}-\left(1-\lambda_{i}(0)\right) f_{i}^{+}\right) \tilde{r}_{i}^{+} \\
= & \sum_{i}\left(g_{i}^{-}+g_{i}^{+}-\left(1+\lambda_{i}(0)\right) f_{i}^{-}+\left(1-\lambda_{i}(0)\right) f_{i}^{+}\right)\left(\tilde{r}_{i}^{-}+\mathcal{O}(1) \delta_{0}\right) \\
& +\mathcal{O}(1) \sum_{i}\left(\left|f_{i}^{-}\right|+\left|f_{i}^{+}\right|+\left|g_{i}^{-}+g_{i}^{+}\right|\right)\left(\left|g_{i}^{-}+\theta_{i}^{+} f_{i}^{-}\right|+\left|g_{i}^{+}+\theta_{i}^{+} f_{i}^{+}\right|\right) \\
& +\mathcal{O}(1) \sum_{i}\left(\left|f_{i}^{-}\right|+\left|f_{i}^{+}\right|+\left|g_{i}^{-}+g_{i}^{+}\right|\right)\left(\left|f_{i}^{-}\right|+\left|f_{i}^{+}\right|\right)\left|\sigma_{i}^{-}-\sigma_{i}^{+}\right|,
\end{aligned}
$$

which can be rewritten as

$$
\begin{aligned}
g_{i}^{-}+g_{i}^{+}= & \left(1+\lambda_{i}(0)\right) f_{i}^{-}-\left(1-\lambda_{i}(0)\right) f_{i}^{+} \\
& +\mathcal{O}(1) \sum_{j}\left(\left|f_{i}^{-}\right|+\left|f_{i}^{+}\right|\right)\left(\left|g_{j}^{-}+\theta_{j}^{+} f_{j}^{-}\right|+\left|g_{j}^{+}+\theta_{j}^{+} f_{j}^{+}\right|\right) \\
& +\sum_{j}\left(\left|f_{j}^{-}\right|+\left|f_{j}^{+}\right|\right)^{2}\left|\sigma_{j}^{-}-\sigma_{j}^{+}\right| .
\end{aligned}
$$

Here and in the following we substitute $\mathcal{O}(1) \delta_{0}$ for quantities depending on the $L^{1}, L^{\infty}$ norm of the variables. Coming back to (5.6) it follows that

$$
\tilde{r}^{+}-\tilde{r}^{-}=\mathcal{O}(1)\left(g_{i}^{-}+\theta_{i}^{-} f_{i}^{-}\right)+\mathcal{O}(1)\left(g_{i}^{+}+\theta_{i}^{+} f_{i}^{+}\right)+\mathcal{O}(1)\left(\left|f_{i}^{-}\right|+\left|f_{i}^{+}\right|\right)\left(\theta_{i}^{+}-\theta_{i}^{-}\right) .
$$

Similarly, from $\tilde{\iota}=\iota^{-}+\iota^{+}=h^{-}-h^{+}$one has that

$$
\begin{aligned}
\iota_{i}^{-}+\iota_{i}^{+}= & \left(1+\lambda_{i}(0)\right) h_{i}^{-}-\left(1-\lambda_{i}(0)\right) h_{i}^{+} \\
& +\mathcal{O}(1) \sum_{j}\left(\left|h_{i}^{-}\right|+\left|h_{i}^{+}\right|\right)\left(\left|g_{j}^{-}+\theta_{j}^{+} f_{j}^{-}\right|+\left|g_{j}^{+}+\vartheta_{j}^{+} f_{j}^{+}\right|\right) \\
& +\mathcal{O}(1) \sum_{j}\left(\left|h_{i}^{-}\right|+\left|h_{i}^{+}\right|\right)\left(\left|f_{j}^{-}\right|+\left|f_{j}^{+}\right|\right)\left|\theta_{j}^{-}-\theta_{j}^{+}\right| .
\end{aligned}
$$

5.2. Source terms of the components of the perturbation $h$. We can now write the source terms for the components $\left(h_{i}^{-}, h_{i}^{+}\right)$. The idea is that, once we have written the these equations, by substituting $h^{ \pm}$with $f^{ \pm}, g^{ \pm}, \iota^{ \pm}$and replacing the source terms $s_{h}^{ \pm}(t, x)$ for $h^{ \pm}$with those for $f, g, \iota$, one obtains the equations satisfied by $\left(f_{i}^{-}, f_{i}^{+}\right),\left(g_{i}^{-}, g_{i}^{+}\right),\left(\iota_{i}^{-}, \iota_{i}^{+}\right)$.

Since $\tilde{r}_{j}^{ \pm}$depends on $f_{j}^{ \pm}, g_{j}^{ \pm}$, during the computations we need clearly to assume to have the equations for $f_{j}^{ \pm}, g_{j}^{ \pm}$written: we thus suppose that each component $f_{j}^{ \pm}$satisfies

$$
\left\{\begin{array}{l}
f_{j, t}^{-}-f_{j, x}^{-}=-\frac{1+\tilde{\lambda}_{j}^{-}}{2} f_{j}^{-}+\frac{1-\tilde{\lambda}_{j}^{+}}{2} f_{j}^{+}+\varsigma_{f, j}^{-}(t, x) \\
f_{j, t}^{+}+f_{j, x}^{+}=\frac{1+\tilde{\lambda}_{j}^{-}}{2} f_{j}^{-}-\frac{1-\tilde{\lambda}_{j}^{+}}{2} f_{j}^{+}+\varsigma_{f, j}^{+}(t, x)
\end{array}\right.
$$

and similarly for $g_{j}^{ \pm}$. The form of the source term $\varsigma_{f}^{ \pm}$is computed by using the form of the source term for $h^{ \pm}$, and replacing $h_{j}^{ \pm}$with $f_{j}^{ \pm}$(or with $g_{j}^{ \pm}$if computing $\varsigma_{g}^{ \pm}$). This assumption will be validated when we obtain that the equations for $\left(h_{j}^{-}, h_{j}^{+}\right)$are of the form $(5.10)$. 
Instead of computing explicitly the source terms $\varsigma_{h}^{-}, \varsigma_{h}^{+}$(this is done in Appendix A), we show how their form can be deduced by using the following ideas:

(1) if there is only one component of $f, g$, let us say the $\bar{i}$-th component $f_{\bar{i}}^{ \pm}, g_{\bar{i}}^{ \pm}$, and

$$
g_{\bar{i}}^{-}+\theta_{\bar{i}}^{-} f_{\bar{i}}^{-}=g_{\bar{i}}^{+}+\theta_{\bar{i}}^{+} f_{\bar{i}}^{+}=0, \quad \theta_{\bar{i}}^{-}=\theta_{\bar{i}}^{+},
$$

then we are locally on a travelling profile of the $\bar{i}$-th family;

(2) on a travelling profile of the $\bar{i}$-th family with speed close to $\lambda_{\bar{i}}(0)$ the decomposition is exact, in the sense that the components $\left(f_{\bar{i}}^{-}, f_{\bar{i}}^{+}\right),\left(g_{\bar{i}}^{-}, g_{\bar{i}}^{+}\right)$satisfy the scalar conservative $2 \times 2$ system

$$
\begin{aligned}
& \left\{\begin{array}{l}
f_{\bar{i}, t}^{-}-f_{\bar{i}, x}^{-}=-\frac{1+\tilde{\lambda}_{\bar{i}}}{2} f_{\bar{i}}^{-}+\frac{1-\tilde{\lambda}_{\bar{i}}}{2} f_{\bar{i}}^{+} \\
f_{\bar{i}, t}^{+}+f_{\bar{i}, x}^{+}=\frac{1+\tilde{\lambda}_{\bar{i}}}{2} f_{\bar{i}}^{-}-\frac{1-\tilde{\lambda}_{\bar{i}}}{2} f_{\bar{i}}^{+}
\end{array}\right. \\
& \left\{\begin{array}{l}
g_{\bar{i}, t}^{-}-g_{\bar{i}, x}^{-}=-\frac{1+\tilde{\lambda}_{\bar{i}}}{2} g_{\bar{i}}^{-}+\frac{1-\tilde{\lambda}_{\bar{i}}}{2} g_{\bar{i}}^{+} \\
g_{\bar{i}, t}^{+}+g_{\bar{i}, x}^{+}=\frac{1+\tilde{\lambda}_{\bar{i}}}{2} g_{\bar{i}}^{-}-\frac{1-\tilde{\lambda}_{\bar{i}}}{2} g_{\bar{i}}^{+}
\end{array}\right.
\end{aligned}
$$

with $\tilde{\lambda}_{\bar{i}}=\tilde{\lambda}_{\bar{i}}^{ \pm}=\tilde{\lambda}_{\bar{i}}\left(u, f^{ \pm} /(1 \pm \sigma), \sigma\right)$ computed by $(4.6)$;

(3) if the perturbations $h, \iota$ have only the $\bar{i}$-th component and are proportional to $f, g$, then they satisfy the same equations (5.12), (5.13);

(4) a part from two terms involving derivatives of $\tilde{r}_{i}^{ \pm}$w.r.t. $f$ and $\sigma$, the source term we need to compute is quadratic and Lipschitz continuous.

We prove now these statements.

Proof. From (5.7), (5.8) we obtain immediately that $r_{\bar{i}}^{-}=r_{\bar{i}}^{+}, u_{t}+\sigma u_{x}=0$. Since $\tilde{r}_{j}^{ \pm}$are unitary vectors, by direct substitution in the equations (2.20), (2.29) for $f, g$ (neglecting the additional source term due to the initial data, which at this level can be chosen arbitrarily) one concludes that $f_{\bar{i}}^{ \pm}, g_{\bar{i}}^{ \pm}$satisfy (5.12), (5.13) of point 2), because of (4.6).

Thus the only point is that the derivatives of $\sigma$ are 0 , because one may suspect that it is possible for the speed to vary, even if the assumptions of point 1) are satisfied. However, by using the equations for $f, g$ it follows $(\theta(x)=x$ under point 1$))$

$$
\begin{aligned}
\sigma_{t}-\sigma_{x} & =\frac{g_{\bar{i}, t}^{-}-g_{\bar{i}, x}^{-}}{f_{\bar{i}}^{-}}-\frac{g_{\bar{i}}^{-}}{f_{\bar{i}}^{-}} \frac{f_{\bar{i}, t}^{-}-f_{\bar{i}, x}^{-}}{f_{\bar{i}}^{-}} \\
& =-\frac{1+\tilde{\lambda}_{\bar{i}}}{2}\left(\frac{g_{\bar{i}}^{-}}{f_{\bar{i}}^{-}}-\frac{g_{\bar{i}}^{-}}{f_{\bar{i}}^{-}} \frac{f_{\bar{i}}^{-}}{f_{\bar{i}}^{-}}\right)+\frac{1-\tilde{\lambda}_{\bar{i}}}{2}\left(\frac{g_{\bar{i}}^{+}}{f_{\bar{i}}^{-}}-\frac{g_{\bar{i}}^{-}}{f_{\bar{i}}^{-}} \frac{f_{\bar{i}}^{+}}{f_{\bar{i}}^{-}}\right)=\frac{1+\tilde{\lambda}_{\bar{i}}}{2} \frac{f_{\bar{i}}^{+}}{f_{\bar{i}}^{-}}\left(\frac{g_{\bar{i}}^{+}}{f_{\bar{i}}^{+}}-\frac{g_{\bar{i}}^{-}}{f_{\bar{i}}^{-}}\right)=0,
\end{aligned}
$$

and similarly $\sigma_{t}+\sigma_{x}=0$. It thus follows that $\sigma_{t}=\sigma_{x}=0$, so that we conclude that $u$ satisfy the travelling wave equations. This proves point 1), 2).

Point 3) is straightforward, while point 4) follows once we observe that the functions in (5.3) are Lipschitz continuous as soon as we do not differentiate them w.r.t. $f$, $\sigma$, i.e. the terms containing the vectors $\tilde{r}_{i, f}^{ \pm}, \tilde{r}_{i, \sigma}^{ \pm}$.

The rest of this section is dedicated to the computation of the form of the source terms.

Since the decomposition (5.3) is only Lipschitz continuous, we study carefully the right hand side of (2.39). We have

$$
\begin{aligned}
h_{t}^{-}-h_{x}^{-}= & \sum_{j}\left(\left(h_{j, t}^{-}-h_{j, x}^{-}\right) \tilde{r}_{j}^{-}+h_{j}^{-}\left(f_{j, t}^{-}-f_{j, x}^{-}\right)\left(\tilde{r}_{j, f}^{-}+\theta_{j, f}^{-} \tilde{r}_{j, \sigma}^{-}\right)+h_{j}^{-}\left(g_{j, t}^{-}-g_{j, x}^{-}\right) \theta_{j, g}^{-} \tilde{r}_{j, \sigma}^{-}\right) \\
& +\sum_{j} h_{j}^{-} D \tilde{r}_{j}^{-}\left(\tilde{u}_{t}-\tilde{u}_{x}\right)+\sum_{j} h_{j}^{-} e^{-t} D \tilde{r}_{j}^{-}\left(u_{0, t}+u_{0, t x}\right), \\
h_{t}^{+}+h_{x}^{+}= & \sum_{j}\left(\left(h_{j, t}^{+}+h_{j, x}^{+}\right) \tilde{r}_{j}^{+}+h_{j}^{+}\left(f_{j, t}^{+}+f_{j, x}^{+}\right)\left(\tilde{r}_{j, f}^{+}+\theta_{j, f}^{+} \tilde{r}_{j, \sigma}^{+}\right)+h_{j}^{+}\left(g_{j, t}^{+}+g_{j, x}^{+}\right) \theta_{j, g}^{+} \tilde{r}_{j, \sigma}^{+}\right) \\
& +\sum_{j} h_{j}^{+} D \tilde{r}_{j}^{+}\left(\tilde{u}_{t}+\tilde{u}_{x}\right)+\sum_{j} h_{j}^{+} e^{-t} D \tilde{r}_{j}^{+}\left(u_{0, t}-u_{0, t x}\right),
\end{aligned}
$$


where we have used $\tilde{u}=u+e^{-t} u_{0, t}$. We thus write the equations for $h$ as

$$
\begin{aligned}
& \sum_{j}\left(h_{j, t}^{-}-h_{j, x}^{-}+\frac{1+\tilde{\lambda}_{j}^{-}}{2} h_{j}^{-}-\frac{1-\tilde{\lambda}_{j}^{+}}{2} h_{j}^{+}\right) \tilde{r}_{j}^{-} \\
& +\sum_{j} h_{j}^{-}\left(f_{j, t}^{-}-f_{j, x}^{-}+\frac{1+\tilde{\lambda}_{j}^{-}}{2} f_{j}^{-}-\frac{1-\tilde{\lambda}_{j}^{+}}{2} f_{j}^{+}\right)\left(\tilde{r}_{j, f}^{-}+\theta_{j, f}^{-} \tilde{r}_{j, \sigma}^{-}\right) \\
& +\sum_{j} h_{j}^{-}\left(g_{j, t}^{-}-g_{j, x}^{-}+\frac{1+\tilde{\lambda}_{j}^{-}}{2} g_{j}^{-}-\frac{1-\tilde{\lambda}_{j}^{+}}{2} g_{j}^{+}\right) \theta_{j, g}^{-} \tilde{r}_{j, \sigma}^{-} \\
& =\tilde{s}_{h}^{-}(t, x)-\sum_{j} h_{j}^{-} e^{-t} D \tilde{r}_{j}^{-}\left(u_{0, t}+u_{0, t x}\right)+s_{h}(t, x), \\
& \sum_{j}\left(h_{j, t}^{+}+h_{j, x}^{+}-\frac{1+\tilde{\lambda}_{j}^{-}}{2} h_{j}^{-}+\frac{1-\tilde{\lambda}_{j}^{+}}{2} h_{j}^{+}\right) \tilde{r}_{j}^{+} \\
& +\sum_{j} h_{j}^{+}\left(f_{j, t}^{+}+f_{j, x}^{+}-\frac{1+\tilde{\lambda}_{j}^{-}}{2} f_{j}^{-}+\frac{1-\tilde{\lambda}_{j}^{+}}{2} f_{j}^{+}\right)\left(\tilde{r}_{j, f}^{+}+\theta_{j, f}^{+} \tilde{r}_{j, \sigma}^{+}\right) \\
& +\sum_{j} h_{j}^{+}\left(g_{j, t}^{+}+g_{j, x}^{+}-\frac{1+\tilde{\lambda}_{j}^{-}}{2} g_{j}^{-}+\frac{1-\tilde{\lambda}_{j}^{+}}{2} g_{j}^{+}\right) \theta_{j, g}^{+} \tilde{r}_{j, \sigma}^{+} \\
& =\tilde{s}_{h}^{+}(t, x)-\sum_{j} h_{j}^{+} e^{-t} D \tilde{r}_{j}^{+}\left(u_{0, t}-u_{0, t x}\right)+s_{h}(t, x),
\end{aligned}
$$

The terms $\tilde{s}_{h}^{ \pm}$(which have a quite complicated form, see Appendix A for details) are quadratic w.r.t. $h$, $f, g$ and linear w.r.t. $h$ : this means that

$$
\tilde{s}_{h}^{ \pm}=\mathcal{O}(1) \sum_{j, k}\left(\left|h_{j}^{-}\right|+\left|h_{j}^{+}\right|\right)\left(\left|f_{k}^{-}\right|+\left|f_{k}^{+}\right|+\left|g_{k}^{-}\right|+\left|g_{k}^{+}\right|\right)+\text {exponentially decaying terms. }
$$

They contain the source terms $\varsigma_{f}, \varsigma_{g}$ for $f, g$.

To simplify their form, first we observe that the terms $\varsigma_{f}, \varsigma_{g}$ can be neglected: in fact, once we prove that without these terms the source terms for $h$ is bounded in $L^{1}([0, T] \times \mathbb{R})$ by $C \delta_{0}\|h\|_{L^{1}}$, then the perturbation due to $\varsigma_{f}, \varsigma_{g}$ is of order

$$
C\left(\left\|\varsigma_{f}\right\|_{L^{1}([0, T] \times \mathbb{R})}+\left\|\varsigma_{f}\right\|_{L^{1}([0, T] \times \mathbb{R})}\right)\|h\|_{L^{\infty}}=C \delta_{0}^{2}\|h\|_{L^{\infty}} \leq C \delta_{0}^{4},
$$

i.e. of higher order w.r.t. $\varsigma_{h}$, which will be proved to be $\mathcal{O}(1) \delta_{0}^{2}$.

Next, we study separately the terms which can be discontinuous, i.e.

(1) the derivative of $\tilde{r}_{j}^{ \pm}$w.r.t. $f_{j}^{ \pm}$, only in the regions where either $\left|\frac{f_{j}^{-}}{f_{j}^{+}}\right| \ll 1$ or $\left|\frac{f_{j}^{+}}{f_{j}^{-}}\right| \ll 1$, i.e. taking into account that at equilibrium $\left(1+\lambda_{j}(0)\right) f_{j}^{-}=\left(1-\lambda_{j}(0)\right) f_{j}^{+}$and $\left(1+\lambda_{j}(0)\right) /\left(1-\lambda_{j}(0)\right) \in$ $[c / 4,4 / c]$

$$
h_{j}^{-} f_{j}^{+} \tilde{r}_{j, f}^{-} \chi\left\{\left|f_{j}^{+}\right| \geq 4 / c\left|f_{j}^{-}\right|\right\}, \quad h_{j}^{+} f_{j}^{-} \tilde{r}_{j, f}^{+} \chi\left\{\left|f_{j}^{-}\right| \geq 4 / c\left|f_{j}^{+}\right|\right\} .
$$

The above terms arise when we are far from equilibrium. Note that for a travelling wave one has $\left(1+\sigma_{j}\right) f_{j}^{-}=\left(1-\sigma_{j}\right) f_{j}^{+}$, and by construction $\sigma$ is close to some $\lambda_{j}(0)$ so that we are certainly far from any travelling profiles. In the remaining regions $f_{j}^{+} \simeq f_{j}^{-}$, and the terms containing $\tilde{r}_{j, f}^{ \pm}$ are again Lipschitz;

(2) with a similar computation to the one in (5.14), the derivative of $\tilde{r}_{j}^{ \pm}$w.r.t. $\sigma$ yields

$$
\frac{1-\tilde{\lambda}_{j}^{+}}{2} h_{j}^{-}\left(f_{j}^{+} \frac{g_{j}^{-}}{f_{j}^{-}}-g_{j}^{+}\right)\left(\theta_{j}^{-}\right)^{\prime} \frac{\tilde{r}_{j, \sigma}^{-}}{f_{j}^{-}}, \quad \frac{1+\tilde{\lambda}_{j}^{+}}{2} h_{j}^{-}\left(f_{j}^{-} \frac{g_{j}^{+}}{f_{j}^{+}}-g_{j}^{-}\right)\left(\theta_{j}^{+}\right)^{\prime} \frac{\tilde{r}_{j, \sigma}^{+}}{f_{j}^{+}} .
$$

These terms are bounded, but may have jumps when $g_{j}^{ \pm}, f_{j}^{ \pm} \simeq 0$. 
The remaining terms in $\tilde{s}_{h}^{ \pm}$are quadratic, Lipschitz continuous w.r.t. $f, g$ and linear w.r.t. $h$. Moreover we know from the discussion at the beginning of this section that these terms are 0 when the following conditions hold for any $j$ fixed:

$$
f_{k}^{ \pm}=g_{k}^{ \pm}=h_{k}^{ \pm}=0 \quad k \neq j, \quad \frac{g_{j}^{-}}{f_{j}^{-}}=\frac{g_{j}^{+}}{f_{j}^{+}} \in\left[-2 \delta_{2}, 2 \delta_{2}\right], \quad \frac{h_{j}^{-}}{f_{j}^{-}}=\frac{h_{j}^{+}}{f_{j}^{+}} .
$$

It is easy to check that the only terms which do not satisfy the first condition are those deriving from $h_{j}^{ \pm} D \tilde{r}_{j}^{ \pm}\left(\tilde{u}_{t} \pm \tilde{u}_{x}\right)$, i.e.

$$
\left|h_{j}^{-}\right|\left(\left|f_{k}^{+}\right|+\left|g_{k}^{+}\right|\right), \quad\left|h_{j}^{+}\right|\left(\left|f_{k}^{-}\right|+\left|g_{k}^{-}\right|\right), \quad j \neq k .
$$

We will here consider the more general terms

$$
\left(\left|h_{j}^{-}\right|+\left|h_{j}^{+}\right|\right)\left(\left|f_{k}^{-}\right|+\left|g_{k}^{-}\right|+\left|f_{k}^{+}\right|+\left|g_{k}^{+}\right|\right) .
$$

Following [5], we call them transversal terms.

We write the distance from the hypersurface defined by the second condition of (5.17) as

$$
\left|g_{j}^{-}+\theta_{j}^{-} f_{j}^{-}\right|+\left|g_{j}^{+}+\theta_{j}^{+} f_{j}^{+}\right|+\left(\left|f_{j}^{+}\right|+\left|f_{j}^{-}\right|\right)\left|\theta_{j}^{-}-\theta_{j}^{+}\right| .
$$

By multiplying this distance by $h$, we have clearly the terms in $\tilde{s}_{h}^{ \pm}$corresponding to the second condition of (5.17), namely

$$
\left(\left|h_{j}^{-}\right|+\left|h_{j}^{+}\right|\right)\left(\left|g_{j}^{-}+\theta_{j}^{-} f_{j}^{-}\right|+\left|g_{j}^{+}+\theta_{j}^{+} f_{j}^{+}\right|+\left(\left|f_{j}^{+}\right|+\left|f_{j}^{-}\right|\right)\left|\theta_{j}^{-}-\theta_{j}^{+}\right|\right) .
$$

These are the non transversal terms.

We can write for the two terms of (5.16)

$$
\begin{aligned}
h_{j}^{-}\left(f_{j}^{+} \frac{g^{-}}{f_{j}^{-}}-g_{j}^{+}\right)= & \mathcal{O}(1) h_{j}^{-} f_{j}^{+}\left(\theta_{j}^{-}-\theta_{j}^{+}\right)+\mathcal{O}(1)\left|h_{j}^{-}\right|\left|f_{j}^{+}\right| \chi\left\{\left|f_{j}^{+} / f_{j}^{-}\right| \notin[c / 4,4 / c]\right\} \\
& +\mathcal{O}(1) h_{j}^{-}\left(g_{j}^{-}+\theta_{j}^{-} f_{j}^{-}\right)+\mathcal{O}(1) h_{j}^{-}\left(g_{j}^{+}+\theta_{j}^{+} f_{j}^{+}\right), \\
h_{j}^{+}\left(f_{j}^{-} \frac{g^{+}}{f_{j}^{+}}-g_{j}^{-}\right)= & \mathcal{O}(1) h_{j}^{+} f_{j}^{-}\left(\theta_{j}^{-}-\theta_{j}^{+}\right)+\mathcal{O}(1)\left|h_{j}^{+}\right|\left|f_{j}^{-}\right| \chi\left\{\left|f_{j}^{+} / f_{j}^{-}\right| \notin[c / 4,4 / c]\right\} \\
& +\mathcal{O}(1) h_{j}^{+}\left(g_{j}^{-}+\theta_{j}^{-} f_{j}^{-}\right)+\mathcal{O}(1) h_{j}^{+}\left(g_{j}^{+}+\theta_{j}^{+} f_{j}^{+}\right) .
\end{aligned}
$$

Thus these terms have the same form of (5.19) and (5.15).

If we were studying the equations for $f, g$ we would have done. In fact the last condition of (5.17) is trivially satisfied if $h_{j}^{ \pm}=f_{j}^{ \pm}$, and if $h_{j}^{ \pm}=g_{j}^{ \pm}$then it reduces to the second condition. In our case we just add the term

$$
\left|h_{j}^{-} f_{j}^{+}-h_{j}^{+} f_{j}^{-}\right|
$$

which accounts for the third condition of (5.17).

To write the final estimate on the source terms for $h$, we need to study also

$$
\begin{aligned}
\int_{0}^{t} e^{\tau-t} D A\left(\tilde{u}_{x} \times \tilde{h}-\tilde{h} \times \tilde{u}_{x}\right) d \tau \leq & C \sum_{j \neq k} \int_{0}^{t} e^{t-\tau}\left(\left|h_{j}^{-}\right|+\left|h_{j}^{+}\right|\right)\left(\left|f_{k}^{+}\right|+\left|f_{k}^{-}\right|\right) d \tau \\
& +C \sum_{j} \int_{0}^{t} e^{\tau-t}\left(\left|h_{j}^{-}\right|+\left|h_{j}^{+}\right|\right)\left(\left|g_{j}^{-}+\theta_{j}^{-} f_{j}^{-}\right|+\left|g_{j}^{+}+\theta_{j}^{+} f_{j}^{+}\right|\right) d \tau \\
& +C \sum_{j} \int_{0}^{t} e^{\tau-t}\left(\left|h_{j}^{-}\right|+\left|h_{j}^{+}\right|\right)\left(\left|f_{j}^{-}\right|+\left|f_{j}^{+}\right|\right)\left|\theta_{j}^{+}-\theta_{j}^{-}\right| d \tau .
\end{aligned}
$$

In the above computation we actually did not use the fact that we are in the conservative variables $u_{x}, h$, since in a next section we are able to estimate all the terms appearing in (5.21). A more refined analysis can show that some terms in (5.21) do not appear in the source $s_{h}$. 
If we denote with $\varsigma_{h, i}^{ \pm}$the total source term of $h_{i}^{ \pm}$, we thus have that the components $h_{i}^{ \pm}$satisfy

$$
\left\{\begin{array}{l}
h_{i, t}^{-}-h_{i, x}^{-}=-\frac{1+\tilde{\lambda}_{i}^{-}}{2} h_{i}^{-}+\frac{1-\tilde{\lambda}_{i}^{+}}{2} h_{i}^{+}+\varsigma_{h, i}^{-}(t, x) \\
h_{i, t}^{+}+h_{i, x}^{+}=\frac{1+\tilde{\lambda}_{i}^{-}}{2} h_{i}^{-}-\frac{1-\tilde{\lambda}_{i}^{+}}{2} h_{i}^{+}+\varsigma_{h, i}^{+}(t, x)
\end{array}\right.
$$

and by means of $(2.40),(5.21)$ we have the estimate

$$
\begin{aligned}
\left|\varsigma_{h, j}^{-}(t, x)\right|,\left|\varsigma_{h, j}^{+}(t, x)\right| \leq & C \sum_{j \neq k}\left(\left|h_{j}^{-}\right|+\left|h_{j}^{+}\right|\right)\left(\left|f_{k}^{-}\right|+\left|g_{k}^{-}\right|+\left|f_{k}^{+}\right|+\left|g_{k}^{+}\right|\right) \\
& +C \sum_{j}\left(\left|h_{j}^{-}\right|+\left|h_{j}^{+}\right|\right)\left(\left|g_{j}^{-}+\theta_{j}^{-} f_{j}^{-}\right|+\left|g_{j}^{+}+\theta_{j}^{+} f_{j}^{+}\right|\right) \\
& +C \sum_{j}\left(\left|h_{j}^{-}\right|+\left|h_{j}^{+}\right|\right)\left(\left|f_{j}^{-}\right|+\left|f_{j}^{+}\right|\right)\left|\theta_{j}^{+}-\theta_{j}^{-}\right| \\
& +C \sum_{j}\left|h_{j}^{-} f_{j}^{+}-f_{j}^{-} h_{j}^{+}\right|+C \delta_{1} e^{-t} \\
& +C \sum_{j}\left(\left|h_{j}^{-}\right|+\left|h_{j}^{+}\right|\right)\left(\left|f_{j}^{-}\right|+\left|f_{j}^{+}\right|\right) \chi\left\{\left|f_{j}^{+} / f_{j}^{-}\right| \notin[c / 4,4 / c]\right\} \\
& +C \sum_{j \neq k} \int_{0}^{t} e^{\tau-t}\left(\left|h_{j}^{-}\right|+\left|h_{j}^{+}\right|\right)\left(\left|f_{k}^{-}\right|+\left|f_{k}^{+}\right|\right) d \tau \\
& +C \sum_{j} \int_{0}^{t} e^{\tau-t}\left(\left|h_{j}^{-}\right|+\left|h_{j}^{+}\right|\right)\left(\left|g_{j}^{-}+\theta_{j}^{-} f_{j}^{-}\right|+\left|g_{j}^{+}+\theta_{j}^{+} f_{j}^{+}\right|\right) d \tau \\
& +C \sum_{j} \int_{0}^{t} e^{\tau-t}\left(\left|h_{j}^{-}\right|+\left|h_{j}^{+}\right|\right)\left(\left|f_{j}^{-}\right|+\left|f_{j}^{+}\right|\right)\left|\theta_{j}^{+}-\theta_{j}^{-}\right| d \tau .
\end{aligned}
$$

We have used again (5.8) when projecting along $\tilde{r}_{i}^{ \pm}$.

Before entering into the computations to estimates the above terms, we observe that

$$
\int_{0}^{T} \int_{\mathbb{R}} \int_{0}^{t} e^{\tau-t} s(\tau, s) d \tau d x d t=\int_{0}^{T} \int_{\mathbb{R}} s(\tau, x) \int_{\tau}^{T} e^{\tau-t} d t d x d \tau=\int_{0}^{T} \int_{\mathbb{R}}\left(1-e^{\tau-T}\right) s(\tau, x) d x d \tau .
$$

Thus, the source terms we need to estimate are the reduced one

$$
\begin{aligned}
\left|\tilde{\varsigma}_{h, i}^{-}(t, x)\right|,\left|\tilde{\varsigma}_{h, i}^{+}(t, x)\right| \leq & C \sum_{j \neq k}\left(\left|h_{j}^{-}\right|+\left|h_{j}^{+}\right|\right)\left(\left|f_{k}^{-}\right|+\left|g_{k}^{-}\right|+\left|f_{k}^{+}\right|+\left|g_{k}^{+}\right|\right) \\
& +C \sum_{j}\left(\left|h_{j}^{-}\right|+\left|h_{j}^{+}\right|\right)\left(\left|g_{j}^{-}+\theta_{j}^{-} f_{j}^{-}\right|+\left|g_{j}^{+}+\theta_{j}^{+} f_{j}^{+}\right|\right) \\
& +C \sum_{j}\left(\left|h_{j}^{-}\right|+\left|h_{j}^{+}\right|\right)\left(\left|f_{j}^{-}\right|+\left|f_{j}^{+}\right|\right)\left|\theta_{j}^{+}-\theta_{j}^{-}\right| \\
& +C \sum_{j}\left|h_{j}^{-} f_{j}^{+}-f_{j}^{-} h_{j}^{+}\right|+C \delta_{1} e^{-t} \\
& +C \sum_{j}\left(\left|h_{j}^{-}\right|+\left|h_{j}^{+}\right|\right)\left(\left|f_{j}^{-}\right|+\left|f_{j}^{+}\right|\right) \chi\left\{\left|f_{j}^{+} / f_{j}^{-}\right| \notin[c / 4,4 / c]\right\} .
\end{aligned}
$$

The constant $C$ depends only on $\delta_{0}$ and the $L^{1}$ norm of $f, g, h$, so that it can be chosen uniformly in the time interval $[0, T]$. In the following it could be proportional to $\delta_{2}^{-1}$, but this constant is independent on the initial data.

Remark 5.1. For $\iota$, one may check that the non local-in-time source term contains also

$$
\sum_{j} \int_{0}^{t} e^{\tau-t}\left|f_{j}^{+} h_{j}^{-}-h_{j}^{+} f_{j}^{-}\right| d \tau .
$$

Clearly this term does not create any problem if we are able to estimate all the terms in (5.24). 
5.3. Equations satisfied by $f, g$. A particular and important case are the equations satisfied by $\left(f_{j}^{-}, f_{j}^{+}\right),\left(g_{j}^{-}, g_{j}^{+}\right)$. For completeness we write them explicitly: by replacing $h$ with $f$ and $\iota$ with $g$ we conclude that the source terms $\varsigma_{f, i}^{ \pm}$of $(5.10)$ can be estimated as

$$
\begin{aligned}
\left|\varsigma_{f, i}^{-}\right|,\left|\varsigma_{f, i}^{+}\right| \leq & C \sum_{j \neq k}\left(\left(\left|f_{j}^{-}\right|+\left|f_{j}^{+}\right|\right)\left(\left|f_{k}^{+}\right|+\left|g_{k}^{+}\right|+\left|f_{k}^{-}\right|+\left|g_{k}^{-}\right|\right)\right) \\
& +C \sum_{j}\left(\left|f_{j}^{-}\right|+\left|f_{j}^{+}\right|\right)\left(\left|g_{j}^{-}+\theta_{j}^{-} f_{j}^{-}\right|+\left|g_{j}^{+}+\theta_{j}^{+} f_{j}^{+}\right|\right) \\
& +C \sum_{j}\left(\left|f_{j}^{-}\right|+\left|f_{j}^{+}\right|\right)^{2}\left|\theta_{j}^{+}-\theta_{j}^{-}\right|+C \delta_{1} e^{-t} \\
& +C \sum_{j}\left(\left|f_{j}^{-}\right|+\left|f_{j}^{+}\right|\right)^{2} \chi\left\{\left|f_{j}^{+} / f_{j}^{-}\right| \notin[c / 4,4 / c]\right\} .
\end{aligned}
$$

Similarly one has

$$
\left\{\begin{array}{l}
g_{i, t}^{-}-g_{i, x}^{-}=-\frac{1+\tilde{\lambda}_{i}^{-}}{2} g_{i}^{-}+\frac{1-\tilde{\lambda}_{i}^{+}}{2} g_{i}^{+}+\varsigma_{g, i}^{-}(t, x) \\
g_{i, t}^{+}+g_{i, x}^{+}=\frac{1+\tilde{\lambda}_{i}^{-}}{2} g_{i}^{-}-\frac{1-\tilde{\lambda}_{i}^{+}}{2} g_{i}^{+}+\varsigma_{g, i}^{+}(t, x)
\end{array}\right.
$$

with

$$
\begin{aligned}
\left|\varsigma_{g, j}^{-}(t, x)\right|,\left|\varsigma_{g, j}^{+}(t, x)\right| \leq & C \sum_{j \neq k}\left(\left(\left|g_{j}^{-}\right|+\left|g_{j}^{+}\right|\right)\left(\left|f_{k}^{+}\right|+\left|g_{k}^{+}\right|+\left|f_{k}^{-}\right|+\left|g_{k}^{-}\right|\right)\right) \\
& +C \sum_{j}\left(\left|g_{j}^{-}\right|+\left|g_{j}^{+}\right|\right)\left(\left|g_{j}^{-}+\theta_{j}^{-} f_{j}^{-}\right|+\left|g_{j}^{+}+\theta_{j}^{+} f_{j}^{+}\right|\right) \\
& +C \sum_{j}\left(\left|g_{j}^{-}\right|+\left|g_{j}^{+}\right|\right)\left(\left|f_{j}^{-}\right|+\left|f_{j}^{+}\right|\right)\left|\theta_{j}^{+}-\theta_{j}^{-}\right| \\
& +C \sum_{j}\left|g_{j}^{-} f_{j}^{+}-f_{j}^{-} g_{j}^{+}\right|+C \delta_{1} e^{-t} \\
& +C \sum_{j}\left(\left|g_{j}^{-}\right|+\left|g_{j}^{+}\right|\right)\left(\left|f_{j}^{-}\right|+\left|f_{j}^{+}\right|\right) \chi\left\{\left|f_{j}^{+} / f_{j}^{-}\right| \notin[c / 4,4 / c]\right\} \\
& +C \sum_{j \neq k} \int_{0}^{t} e^{\tau-t}\left(\left|g_{j}^{-}\right|+\left|g_{j}^{+}\right|\right)\left(\left|f_{k}^{-}\right|+\left|f_{k}^{+}\right|+\left|g_{j}^{-}\right|+\left|g_{j}^{+}\right|\right) d \tau \\
& +C \sum_{j} \int_{0}^{t} e^{\tau-t}\left(\left|g_{j}^{-}\right|+\left|g_{j}^{+}\right|\right)\left(\left|g_{j}^{-}+\theta_{j}^{-} f_{j}^{-}\right|+\left|g_{j}^{+}+\theta_{j}^{+} f_{j}^{+}\right|\right) d \tau \\
& +C \int_{0}^{t} e^{\tau-t}\left(\left|g_{j}^{-}\right|+\left|g_{j}^{+}\right|\right)\left(\left|f_{j}^{-}\right|+\left|f_{j}^{+}\right|\right)\left|\theta_{j}^{+}-\theta_{j}^{-}\right| d \tau .
\end{aligned}
$$

5.4. Simplification of the source terms. We now describe the line of the proof of Theorem 1.1.

We assume that in the time interval $[0, T]$ the various components of the source terms for $f, g, h, \iota$ are bounded by $\delta_{0}$. By the results of Section 3 this is correct if $T$ is sufficiently small. Since by the $L^{1}$ contraction of $2 \times 2$ Jin-Xin model we have

$$
\left\|h_{i}^{-}(t)\right\|_{L^{1}}+\left\|h_{i}^{+}(t)\right\|_{L^{1}} \leq\left\|h_{i}^{-}(0)\right\|_{L^{1}}+\left\|h_{i}^{+}(0)\right\|_{L^{1}}+\int_{0}^{t}\left(\left|\tilde{s}_{h}^{-}(\tau)\right|+\left|\tilde{s}_{h}^{+}(\tau)\right|\right) d \tau \leq 2 \delta_{1}+2 \delta_{0}<4 \delta_{0}
$$

the $L^{1}$ norm of the components is bounded uniformly in $[0, T]$. We can assume that $T$ is the first time where the $L^{1}$ norm of some component reaches $4 \delta_{0}$. If we can prove that from $\left\|h_{j}^{ \pm}\right\|_{L^{1}} \leq 4 \delta_{0} \forall j$ it follows that the source remains uniformly small and less than $\delta_{0}$, then from (5.28) at time $T$ the components of $h$ should have a $L^{1}$ norm less than $4 \delta_{0}$, so that the solution can be prolonged for a small $\delta t$ with $L^{1}$ norm still less than $4 \delta_{0}$ : we thus have a contradiction. As a consequence $T=\infty$ : the solution exists for all $t \geq 0$ and has uniform $L^{1}$ norm (BV norm for $u$ ).

While the source terms of (5.24) have been already been simplified, in this section we put in evidence which are the basic terms to be estimated. The idea is similar to (5.15), where a particular source term 
appears only in some region where the components satisfy some inequality. To be precise, the various situations we consider are the following

(1) regions where the speed $g_{j}^{ \pm} / f_{j}^{ \pm}$is greater than $2 \delta_{2}$;

(2) regions where the two components $h_{j}^{-}, h_{j}^{+}$have different sign;

(3) regions where the ratio $h_{j}^{-} / h_{j}^{+}$is much different from $\left(1-\lambda_{j}(0)\right) /\left(1+\lambda_{j}(0)\right)$.

The first simplification is thus the observation that in the regions where $g_{j}^{-}+\theta_{j}^{-} f_{j}^{-} \neq 0$ we have the simple estimate

$$
\left|g_{j}^{-}+\theta_{j}^{-} f_{j}^{-}\right| \leq C \delta_{2}^{-1}\left|g_{j}^{-}\right| \leq C\left|g_{j}^{-}\right|
$$

Similarly for $g_{j}^{+}+\theta_{j}^{+} f_{j}^{+}$,

$$
\left|g_{j}^{+}+\theta_{j}^{+} f_{j}^{+}\right| \leq C \delta_{2}^{-1}\left|g_{j}^{+}\right| \leq C\left|g_{j}^{+}\right| .
$$

As we noted before, $\delta_{2}$ does not depend on $\delta_{0}$, so that $\delta_{2}^{-1}$ can be included in the constant $C$.

The second observation is that if the sign of the components +- of $f, g, h$ is different, we can reduce the source terms containing them as

$$
C^{2} \delta_{0}^{2}\left|f_{j}^{-}-f_{j}^{+}\right|, \quad C^{2} \delta_{0}^{2}\left|h_{j}^{-}-h_{j}^{+}\right|, \quad C^{2} \delta_{0}^{2}\left|h_{j}^{-}-h_{j}^{+}\right| .
$$

We have used the $L^{1}$ estimate of the derivative of the components, Theorem 3.1.

The transversal terms do not need any transformation, since we can estimate them as they are. Also the terms

$$
\left|h_{j}^{-} f_{j}^{+}-f_{j}^{-} h_{j}^{+}\right|, \quad C \delta_{1} e^{-t},
$$

will be estimated directly (the last one is trivial, of course).

Next we observe that from (5.7) (now $g_{j}^{-}$and $g_{j}^{+}$have the same sign), when $g_{j}^{-}+\theta_{j}^{-} f_{j}^{-} \neq 0$ or $g_{j}^{+}+\theta_{j}^{+} f_{j}^{+} \neq 0$ we have that

$$
\left|\frac{f_{j}^{-}}{1-\lambda_{j}(0)}-\frac{f_{j}^{+}}{1+\lambda_{j}(0)}\right| \geq 2 \delta_{2}-C \delta_{0}^{4} \geq \frac{3 \delta_{2}}{2} .
$$

Thus, in the regions where $g_{j}^{-}+\theta_{j}^{-} f_{j}^{-} \neq 0$ or $g_{j}^{+}+\theta_{j}^{+} f_{j}^{+} \neq 0$, we can write

$$
\begin{aligned}
\left(h_{j}^{+}+h_{j}^{-}\right)\left(g_{j}^{+}+g_{j}^{-}\right) \leq & \left(\left(h_{j}^{-}+h_{j}^{+}\right)^{2}+\left(g_{j}^{-}+g_{j}^{+}\right)^{2}\right) \chi\left\{\left(\frac{h_{j}^{+}}{h_{j}^{-}}-\frac{1+\lambda_{j}(0)}{1+\lambda_{j}(0)}\right) \notin\left[-5 \delta_{2} / 4,5 \delta_{2} / 4\right]\right\} \\
& +C\left(\left|h_{j}^{-} f_{j}^{-}\right|+\left|h_{j}^{+} f_{j}^{+}\right|\right) \chi\left\{\left(\frac{h_{j}^{+}}{h_{j}^{-}}-\frac{1+\lambda_{j}(0)}{1+\lambda_{j}(0)}\right) \in\left[-5 \delta_{2} / 4,5 \delta_{2} / 4\right]\right\} \\
\leq & \left(\left(h_{j}^{-}+h_{j}^{+}\right)^{2}+\left(g_{j}^{-}+g_{j}^{+}\right)^{2}\right) \chi\left\{\left(\frac{h_{j}^{+}}{h_{j}^{-}}-\frac{1+\lambda_{j}(0)}{1+\lambda_{j}(0)}\right) \notin\left[-5 \delta_{2} / 4,5 \delta_{2} / 4\right]\right\} \\
& +C\left(\left|h_{j}^{+} f_{j}^{-}-h_{j}^{-} f_{j}^{+}\right|\right) \chi\left\{\left(\frac{h_{j}^{+}}{h_{j}^{-}}-\frac{1+\lambda_{j}(0)}{1+\lambda_{j}(0)}\right) \in\left[-5 \delta_{2} / 4,5 \delta_{2} / 4\right]\right\} .
\end{aligned}
$$

In the last inequality we notice that in the region

$$
\left\{\left(\frac{h_{j}^{+}}{h_{j}^{-}}-\frac{1+\lambda_{j}(0)}{1+\lambda_{j}(0)}\right) \in\left[-5 \delta_{2} / 4,5 \delta_{2} / 4\right],\left(\frac{f_{j}^{+}}{f_{j}^{-}}-\frac{1+\lambda_{j}(0)}{1+\lambda_{j}(0)}\right) \notin\left[-3 \delta_{2} / 2,3 \delta_{2} / 2\right]\right\}
$$

one has

$$
\left|h_{j}^{-} f_{j}^{+}-h_{j}^{+} f_{j}^{-}\right|=\left|h_{j}^{-} f_{j}^{-}\right|\left|\frac{f_{j}^{+}}{f_{j}^{-}}-\frac{h_{j}^{+}}{h_{j}^{-}}\right| \geq \frac{\delta_{2}}{4}\left|h_{j}^{-} f_{j}^{-}\right|,
$$

and similarly for $h_{j}^{+} f_{j}^{+}$. The constant $\delta_{2}^{-1}$ is collected in $C$ as before. 
For the non transversal terms $\left(h_{j}^{-}+h_{j}^{+}\right)\left(f_{j}^{-}+f_{j}^{+}\right)\left(\theta_{j}^{-}-\theta_{j}^{+}\right)$, we have

$$
\begin{aligned}
\left|h_{j}^{-} f_{j}^{-}\left(\theta_{j}^{-}-\theta_{j}^{+}\right)\right| \leq C\left|h_{j}^{-}\left(f_{j}^{-} / f_{j}^{+}\right) g_{j}^{+}-h_{j}^{-} g_{j}^{-}\right| \\
=C\left|\left(f_{j}^{-} / f_{j}^{+}\right)\left(g_{j}^{+} h_{j}^{-}-h_{j}^{+} g_{j}^{-}\right)\right|+C\left|\left(h_{j}^{+}\left(f_{j}^{-} / f_{j}^{+}\right)-h_{j}^{-}\right) g_{j}^{-}\right| \\
=C\left|\left(f_{j}^{-} / f_{j}^{+}\right)\left(g_{j}^{+} h_{j}^{-}-h_{j}^{+} g_{j}^{-}\right)\right|+C\left|\left(g_{j}^{-} / f_{j}^{-}\right)\left(f_{j}^{-} / f_{j}^{+}\right)\left(h_{j}^{+} f_{j}^{-}-f_{j}^{+} h_{j}^{-}\right)\right|, \\
\left|h_{j}^{+} f_{j}^{-}\left(\theta_{j}^{-}-\theta_{j}^{-}\right)\right| \leq C\left|h_{j}^{+}\left(f_{j}^{-} / f_{j}^{+}\right) g_{j}^{+}-h_{j}^{+} g_{j}^{-}\right| \\
=C\left|\left(\left(f_{j}^{-} / f_{j}^{+}\right) h_{j}^{+}-h_{j}^{-}\right) g_{j}^{+}\right|+C\left|h_{j}^{+} g_{j}^{-}-g_{j}^{+} h_{j}^{-}\right| \\
=C\left|\left(g_{j}^{+} / f_{j}^{+}\right)\left(f_{j}^{-} h_{j}^{+}-h_{j}^{-} f_{j}^{+}\right)\right|+C\left|h_{j}^{+} g_{j}^{-}-g_{j}^{+} h_{j}^{-}\right|,
\end{aligned}
$$

and similarly

$$
\begin{gathered}
\left|h_{j}^{-} f_{j}^{+}\left(\theta_{j}^{-}-\sigma_{j}^{+}\right)\right| \leq C\left|\left(g_{j}^{-} / f_{j}^{-}\right)\left(f_{j}^{-} h_{j}^{+}-h_{j}^{-} f_{j}^{+}\right)\right|+C\left|h_{j}^{+} g_{j}^{-}-g_{j}^{+} h_{j}^{-}\right|, \\
\left|h_{j}^{+} f_{j}^{+}\left(\theta_{j}^{+}-\theta_{j}^{-}\right)\right| \leq C\left|\left(f_{j}^{+} / f_{j}^{-}\right)\left(g_{j}^{+} h_{j}^{-}-h_{j}^{+} g_{j}^{-}\right)\right|+C\left|\left(g_{j}^{+} / f_{j}^{+}\right)\left(f_{j}^{+} / f_{j}^{-}\right)\left(h_{j}^{+} f_{j}^{-}-f_{j}^{+} h_{j}^{-}\right)\right| .
\end{gathered}
$$

Thus if $\left|f_{j}^{+} / f_{j}^{-}\right|,\left|f_{j}^{-} / f_{j}^{+}\right|,\left|g_{j}^{-} / f_{j}^{-}\right|,\left|g_{j}^{+} / f_{j}^{+}\right|$are bounded, then we obtain

$$
\left|h_{j}^{-}+h_{j}^{+}\right|\left|f_{j}^{-}+f_{j}^{+}\right|\left|\theta_{j}^{+}-\theta_{j}^{-}\right| \leq C\left(\left|h^{+} f^{-}-f^{+} h^{-}\right|+\left|h^{+} g^{-}-g^{+} h^{-}\right|\right) .
$$

If $\left|g_{j}^{-} / f_{j}^{-}\right| \ll 1$ or $\left|g_{j}^{+} / f_{j}^{+}\right| \ll 1$ (only one case can occur), then by (5.7) we have $\left|f_{j}^{+} / f_{j}^{-}\right| \gg 1$ or $\left|f^{-} / f_{j}^{+}\right| \gg 1$. In this case, if $\left|h_{j}^{+} / h_{j}^{-}\right| \simeq 1$, then as in (5.30) we obtain that

$$
\left|f_{j}^{-} h_{j}^{-}\right|+\left|f_{j}^{+} h_{j}^{+}\right| \leq C \delta_{2}^{-1}\left|h_{j}^{-} f_{j}^{+}-f_{j}^{-} h_{j}^{+}\right|
$$

so that we obtain again for $\left|h_{j}^{+} / h_{j}^{-}\right| \simeq 1$ that

$$
\left|h_{j}^{-}+h_{j}^{+}\right|\left|f_{j}^{-}+f_{j}^{+}\right|\left|\theta_{j}^{-}-\theta_{j}^{+}\right| \leq C\left(\left|h_{j}^{-} f_{j}^{-}\right|+\left|h_{j}^{+} f_{j}^{+}\right|\right) \leq C\left|f^{-} h^{+}-h^{-} f^{+}\right| .
$$

The ultimate case is thus when $\left|f_{j}^{+} / f_{j}^{-}\right|,\left|h_{j}^{+} / h_{j}^{-}\right| \gg 1$ (or $\ll 1$ ), in this case we have

$$
\left|h_{j}^{-}+h_{j}^{+}\right|\left|f_{j}^{-}+f_{j}^{+} \| \theta_{j}^{+}-\theta_{j}^{-}\right| \leq C\left(\left(h_{j}^{-}+h_{j}^{+}\right)^{2}+\left(f_{j}^{-}+f_{j}^{+}\right)^{2}\right) \leq C\left(\left(h_{j}^{-}+h_{j}^{+}\right)^{2}+\left(g_{j}^{-}+g_{j}^{+}\right)^{2}\right),
$$

where in the last inequality we use again (5.7).

The form of the source terms $\tilde{\varsigma}_{h, i}^{ \pm}$can be thus rewritten as

$$
\begin{aligned}
\left|\tilde{\varsigma}_{h, i}^{-}(t, x)\right|,\left|\tilde{\varsigma}_{h, i}^{+}(t, x)\right| \leq & C \sum_{j \neq k}\left(\left|h_{j}^{-}\right|+\left|h_{j}^{+}\right|\right)\left(\left|f_{k}^{+}\right|+\left|g_{k}^{+}\right|+\left|f_{k}^{-}\right|+\left|g_{k}^{-}\right|\right) \\
& +C \sum_{j}\left|h_{j}^{-} f_{j}^{+}-f_{j}^{-} h_{j}^{+}\right|+C \sum_{j}\left|h_{j}^{-} g_{j}^{+}-g_{j}^{-} h_{j}^{+}\right|+C \sum_{j}\left|g_{j}^{-} f_{j}^{+}-f_{j}^{-} g_{j}^{+}\right| \\
& +C \sum_{j}\left|h_{j}^{-}+h_{j}^{+}\right|^{2} \chi\left\{\left(\frac{h_{j}^{+}}{h_{j}^{-}}-\frac{1+\lambda_{j}(0)}{1+\lambda_{j}(0)}\right) \notin\left[-5 \delta_{2} / 4,5 \delta_{2} / 4\right]\right\} \\
& +C \sum_{j}\left(\left|f_{j}^{-}+f_{j}^{+}\right|^{2}+\left|g_{j}^{-}+g_{j}^{+}\right|^{2}\right) \chi\left\{\left(\frac{f_{j}^{+}}{f_{j}^{-}}-\frac{1+\lambda_{j}(0)}{1+\lambda_{j}(0)}\right) \notin\left[-5 \delta_{2} / 4,5 \delta_{2} / 4\right]\right\} \\
& +C \delta_{0}^{2} \sum_{j}\left|h_{j}^{-}-h_{j}^{+}\right| \chi\left\{h_{j}^{-} h_{j}^{+}<0\right\}+C \delta_{0}^{2} \sum_{j}\left|f_{j}^{-}-f_{j}^{+}\right| \chi\left\{f_{j}^{-} f_{j}^{+}<0\right\}
\end{aligned}
$$

$$
+C \delta_{0}^{2} \sum_{j}\left|g_{j}^{-}-g_{j}^{+}\right| \chi\left\{g_{j}^{-} g_{j}^{+}<0\right\}+C \delta_{1} e^{-t}
$$

In the following we will replace $\tilde{\lambda}_{i}^{+}$with $\tilde{\lambda}_{i}^{-}$, because their difference is of order of $\tilde{\varsigma}_{h, j}^{ \pm}$and can be included into it. 


\section{INTERACTION ESTIMATES}

In this section we prove that under the assumptions of regularity and $\|h\|_{L^{1}} \leq 4 \delta_{0}$, then the various terms appearing in (5.31) are of order $C \delta_{0}^{2}$.

We divide these computations in 4 parts. Each type of source term correspond to a different functional we have to use. The terms are:

transversal terms: these are the terms

$$
\left(\left|h_{j}^{-}\right|+\left|h_{j}^{+}\right|\right)\left(\left|f_{k}^{-}\right|+\left|f_{k}^{+}\right|+\left|g_{k}^{-}\right|+\left|g_{k}^{+}\right|\right) .
$$

These terms correspond to the interaction among waves of different families;

non transversal terms: these term correspond to interaction of waves of the same family. They are

$$
\left|h_{j}^{-} f_{j}^{+}-f_{j}^{-} h_{j}^{+}\right|, \quad\left|h_{j}^{-} g_{j}^{+}-g_{j}^{-} h_{j}^{+}\right|, \quad\left|g_{j}^{-} f_{j}^{+}-f_{j}^{-} g_{j}^{+}\right| ;
$$

energy terms: these terms appear when one wave in the decomposition has a speed too much different w.r.t. $\lambda(0)$, or equivalently $f_{j}^{-} / f_{j}^{+} \not 1$ and similarly for $g_{j}^{ \pm}, h_{j}^{ \pm}$. We just write the term for $h$, the other being similar:

$$
\left|h_{j}^{-}+h_{j}^{+}\right|^{2} \chi\left\{\left(\frac{h_{j}^{+}}{h_{j}^{-}}-\frac{1+\lambda_{j}(0)}{1+\lambda_{j}(0)}\right) \notin\left[-5 \delta_{2} / 4,5 \delta_{2} / 4\right]\right\}
$$

opposite sign terms: these terms correspond to the fact that if $h_{i}^{-}, h_{i}^{+}$have different sign, then their $L^{1}$ norm is decaying, and for $h$ they have the form

$$
\left|h_{j}^{-}-h_{j}^{+}\right| \chi\left\{h_{j}^{-} h_{j}^{+}<0\right\} .
$$

We now analyze the different types, starting with the last one, which is the easiest.

6.1. Opposite sign terms. We just need to repeat the computations of (3.6). We consider the model scheme

$$
\left\{\begin{array}{c}
z_{t}^{-}-z_{x}^{-}=-\frac{1+a(t, x)}{2} z^{-}+\frac{1-a(t, x)}{2} z^{+}+s^{-}(t, x) \\
z_{t}^{+}-z_{x}^{+}=\frac{1+a(t, x)}{2} z^{-}-\frac{1-a(t, x)}{2} z^{+}+s^{+}(t, x)
\end{array}\right.
$$

with the assumption $|a(t, x)| \leq 1-c,\left\|s^{ \pm}\right\|_{L^{1}([0, T] \times \mathbb{R})} \leq \delta_{0}$. Then, by a slight variation of (3.6) one obtains

$$
\frac{d}{d t}\left(\left\|z^{-}\right\|_{L^{1}}+\left\|z^{+}\right\|_{L^{1}}\right) \leq-c \int_{z^{-} z^{+}<0}\left|z^{-}(t, x)-z^{+}(t, x)\right|+\int_{\mathbb{R}}\left|s^{-}(t, x)\right|+\left|s^{+}(t, x)\right| d x
$$

so that integrating in time and assuming $\left\|z^{ \pm}(t=0)\right\|_{L^{1}} \leq \delta_{0}$, one has

$$
\int_{0}^{T} \int_{z^{-} z^{+}<0}\left|z^{-}(t, x)\right|+\left|z^{+}(t, x)\right| d x \leq 2 \delta_{0}+2 \delta_{0} \leq 4 \delta_{0} .
$$

In particular we obtain the estimates

$$
\int_{0}^{T} \int_{h_{j}^{-} h_{j}^{+}}\left|h_{j}^{-}-h_{j}^{+}\right| d x, \int_{0}^{T} \int_{h_{j}^{-} h_{j}^{+}}\left|h_{j}^{-}-h_{j}^{+}\right| d x, \int_{0}^{T} \int_{h_{j}^{-} h_{j}^{+}}\left|h_{j}^{-}-h_{j}^{+}\right| d x \leq 4 \delta_{0},
$$

so that the last three terms of $(5.31)$ are of order $C \delta_{0}^{3}$.

6.2. Transversal terms. These terms are estimated by means of an interaction functional, which is the extension of the Glimm interaction functional for transversal families to the Jin-Xin relaxation model. The method to find this functional is by using complex analysis, and it is developed in [2], showing that these terms are essential linear terms. For a probabilistic approach, see [15].

Here we write only the final form of the functional, and check that it is decreasing and bounds the term of (5.18). Next, by a simple argument, we prove how to estimate all the transversal terms appearing in $(5.31)$. 
Given two constant $a_{1}, a_{2}$, with $-1+c \leq a_{1}<a_{2} \leq 1-c$, define the two $4 \times 4$ tensors $P_{i j k \ell}^{-}(x)$, $P_{i j k \ell}^{+}(x), i, j, k, \ell=-,+, x \in \mathbb{R}$, by

$$
\begin{aligned}
{\left[P_{i j}^{-}(x)\left(\begin{array}{c}
v_{1}^{-} \\
v_{1}^{+}
\end{array}\right) \times\left(\begin{array}{c}
v_{2}^{-} \\
v_{2}^{+}
\end{array}\right)\right] \doteq } & \frac{1}{a_{2}-a_{1}}\left(\frac{1+a_{1}}{2+a_{1}+a_{2}} v_{1}^{-}+\frac{1-a_{1}}{2-a_{1}-a_{2}} v_{1}^{+}\right) \\
& \cdot\left(\frac{1+a_{2}}{2+a_{1}+a_{2}} v_{2}^{-}+\frac{1-a_{2}}{2-a_{1}-a_{2}} v_{2}^{+}\right) \\
& \cdot \frac{1}{4}\left[\begin{array}{cc}
\left(2-a_{1}-a_{2}\right)^{2} & 4-\left(a_{1}+a_{2}\right)^{2} \\
4-\left(a_{1}+a_{2}\right)^{2} & \left(2+a_{1}+a_{2}\right)^{2}
\end{array}\right] \exp \left\{-\frac{2\left(a_{1}-a_{2}\right)}{4-\left(a_{1}+a_{2}\right)^{2}} x_{0}\right\},
\end{aligned}
$$

$$
\left[P_{i j}^{+}(x)\left(\begin{array}{c}
v_{1}^{-} \\
v_{1}^{+}
\end{array}\right) \times\left(\begin{array}{c}
v_{2}^{-} \\
v_{2}^{+}
\end{array}\right)\right] \doteq \frac{1}{a_{2}-a_{1}}\left(v_{1}^{-}+v_{1}^{+}\right)\left(v_{2}^{-}+v_{2}^{+}\right) \frac{1}{4}\left[\begin{array}{cc}
\left(1-a_{1}\right)\left(1-a_{2}\right) & \left(1-a_{1}\right)\left(1+a_{2}\right) \\
\left(1+a_{1}\right)\left(1-a_{2}\right) & \left(1+a_{1}\right)\left(1+a_{2}\right)
\end{array}\right] .
$$

Define finally the weight function $P_{i j k \ell}(x)$ by

$$
P_{i j k \ell}(x) \doteq \begin{cases}P_{i j k \ell}^{-}(x) & x \leq 0 \\ P_{i j k \ell}^{+}(x) & x>0\end{cases}
$$

and the interaction functional $Q(x)$ on the two vector valued functions $z_{1}, z_{2}: \mathbb{R} \mapsto \mathbb{R}^{2}$ by

$$
Q\left(\left(z_{1}^{-}, z_{1}^{+}\right),\left(z_{2}^{+}, z_{2}^{+}\right)\right) \doteq \iint_{\mathbb{R}^{2}}\left(P_{12}(x-y)+P_{21}(x-y)\right)\left(\begin{array}{c}
\left|z_{1}^{-}(x)\right| \\
\left|z_{1}^{+}(x)\right|
\end{array}\right) \times\left(\begin{array}{c}
\left|z_{2}^{-}(y)\right| \\
\left|z_{2}^{+}(y)\right|
\end{array}\right) d x d y .
$$

Remark 6.1. As was observed in [2], the meaning of the weight function $P_{i j k \ell}(x)$ is the following. Consider two schemes (6.1) for $z_{1}, z_{2}$, with source term equal to 0 and constant average speed $a_{1}, a_{2}$ satisfying the above assumptions. Then if the initial data are

$$
z_{1}^{k}(0, x)=\delta\left(x-x_{0}\right), \quad z_{2}^{\ell}(0, x)=\delta(x), \quad x_{0} \neq 0,
$$

and the other two components are 0 , we have that the following equality holds

$$
\int_{0}^{+\infty} \int_{\mathbb{R}} z_{1}^{i}(t, x) z_{2}^{j}(t, x) d x d t=P_{i j k \ell}\left(x_{0}\right) .
$$

Thus in (6.7) we are just computing all the interaction among the +- components, with initial data $z_{1}$, $z_{2}$.

To show how this functional works, we consider two systems of the form

$$
\begin{gathered}
\left\{\begin{array}{l}
z_{1, t}^{-}-z_{1, x}^{-}=-\frac{1+a_{1}(t, x)}{2} z_{1}^{-}+\frac{1-a_{1}(t, x)}{2} z_{1}^{+}+s_{1}^{-}(t, x) \\
z_{1, t}^{+}+z_{1, x}^{+}=\frac{1+a_{1}(t, x)}{2} z_{1}^{-}-\frac{1-a_{1}(t, x)}{2} z_{1}^{+}+s_{1}^{+}(t, x)
\end{array}\right. \\
\left\{\begin{array}{l}
z_{2, t}^{-}-z_{2, x}^{-}=-\frac{1+a_{2}(t, x)}{2} z_{2}^{-}+\frac{1-a_{2}(t, x)}{2} z_{2}^{+}+s_{2}^{-}(t, x) \\
z_{2, t}^{+}+z_{2, x}^{+}=\frac{1+a_{2}(t, x)}{2} z_{2}^{-}-\frac{1-a_{2}(t, x)}{2} z_{2}^{+}+s_{2}^{+}(t, x)
\end{array}\right.
\end{gathered}
$$

with the assumptions

$$
\left|a_{1}(t, x)\right|,\left|a_{2}(t, x)\right| \leq 1-c, \quad \min _{s, t, s, y}\left\{a_{2}(s, x)-a_{1}(t, y)\right\} \geq c>0, \quad \int_{0}^{t} \int_{\mathbb{R}}\left|s_{1}^{ \pm}\right|+\left|s_{2}^{ \pm}\right| d x d t \leq 2 \delta_{0} .
$$

Define

$$
a_{1}=\max \lambda_{1}, \quad a_{2}=\min \lambda_{2},
$$

and consider the functional $Q$ of (6.7) computed with $a_{1}, a_{2}$. 
Essentially we need to analyze the behavior of the functional constructed by means of the projectors

$$
\begin{aligned}
& \mathcal{L}(x)\left(\begin{array}{c}
v_{1}^{-} \\
v_{1}^{+}
\end{array}\right) \times\left(\begin{array}{c}
v_{2}^{-} \\
v_{2}^{+}
\end{array}\right)= \\
& \begin{cases}\frac{4-\left(a_{1}+a_{2}\right)^{2}}{2\left(a_{2}-a_{1}\right)}\left(\frac{1+a_{1}}{2+a_{1}+a_{2}} v_{1}^{-}+\frac{1-a_{1}}{2-a_{1}-a_{2}} v_{1}^{+}\right)\left(\frac{1+a_{2}}{2+a_{1}+a_{2}} v_{2}^{-}+\frac{1-a_{2}}{2-a_{1}-a_{2}} v_{2}^{+}\right) e^{-\frac{2\left(a_{1}-a_{2}\right)}{4-\left(a_{1}+a_{2}\right)^{2}} x_{0}} & x \leq 0 \\
\frac{1-a_{1} a_{2}}{2\left(a_{2}-a_{1}\right)}\left(v_{1}^{-}+v_{1}^{+}\right)\left(v_{2}^{-}+v_{2}^{+}\right) & x>0\end{cases}
\end{aligned}
$$

Denote for simplicity

$$
\begin{gathered}
A_{1} \doteq\left[\begin{array}{cc}
-\frac{1+a_{1}}{2} & \frac{1-a_{1}}{2} \\
\frac{1+a_{1}}{2} & -\frac{1-a_{1}}{2}
\end{array}\right], \quad A_{2} \doteq\left[\begin{array}{cc}
-\frac{1+a_{2}}{2} & \frac{1-a_{2}}{2} \\
\frac{1+a_{2}}{2} & -\frac{1-a_{2}}{2}
\end{array}\right], \quad \Lambda \doteq\left[\begin{array}{cc}
-1 & 0 \\
0 & 1
\end{array}\right], \quad R=\left[\begin{array}{cc}
1 & 1 \\
-1 & -1
\end{array}\right], \\
L_{1}=\left(\frac{1+a_{1}}{2+a_{1}+a_{2}}, \frac{1-a_{1}}{2-a_{1}-a_{2}}\right), \quad L_{2}=\left(\frac{1+a_{2}}{2+a_{1}+a_{2}}, \frac{1-a_{2}}{2-a_{1}-a_{2}}\right), \quad \alpha=\frac{2\left(a_{1}-a_{2}\right)}{4-\left(a_{1}+a_{2}\right)^{2}}
\end{gathered}
$$

By direct differentiation of the reduced functional

$\tilde{Q}(t) \doteq \iint_{\mathbb{R}^{2}} \mathcal{L}(x-y)\left(\begin{array}{c}\left|v_{1}^{-}(t, x)\right| \\ \left|v_{1}^{+}(t, x)\right|\end{array}\right) \times\left(\begin{array}{c}\left|v_{2}^{-}(t, y)\right| \\ \left|v_{2}^{+}(t, y)\right|\end{array}\right) d x d y$

$$
=\frac{4-\left(a_{1}+a_{2}\right)^{2}}{2\left(a_{2}-a_{1}\right)} \iint_{x<y}\left\langle L_{1},\left|z_{1}(x)\right|\right\rangle\left\langle L_{2},\left|z_{2}(y)\right|\right\rangle e^{-\alpha(x-y)} d x d y+\frac{1-a_{1} a_{2}}{2\left(a_{2}-a_{1}\right)} \iint_{x>y}\left|z_{1}(x)\right|\left|z_{2}(y)\right| d x d y,
$$

one obtains by the $L^{1}$ contraction

$$
\begin{aligned}
\frac{d \tilde{Q}}{d t} \leq & \iint_{\mathbb{R}^{2}} L(x-y)\left(-\Lambda\left|z_{1}\right|_{x}+A_{1}\left|z_{1}(x)\right|-\left(a_{1}(x)-a_{1}\right) R\left|z_{1}(x)\right|+\left|s_{1}(x)\right|\right) \times\left|z_{2}(y)\right| d x d y \\
& +\iint_{\mathbb{R}^{2}} L(x-y)\left|z_{1}(x)\right| \times\left(-\Lambda\left|z_{2}\right|_{y}+A_{2}\left|z_{2}(y)\right|-\left(a_{2}(t, y)-a_{2}\right) R\left|z_{2}(y)\right|+\left|s_{2}(y)\right|\right) d x d y .
\end{aligned}
$$

We have the following equalities (which can be used to define $L_{1}, L_{2}$ and $\left.\alpha, \varpi\right)$ :

so that it follows that

$$
\begin{gathered}
L_{1}\left(\begin{array}{c}
1 \\
-1
\end{array}\right)=\frac{2\left(a_{1}-a_{2}\right)}{4-\left(a_{1}+a_{2}\right)^{2}}, \quad L_{2}\left(\begin{array}{c}
1 \\
-1
\end{array}\right)=\frac{2\left(a_{2}-a_{1}\right)}{4-\left(a_{1}+a_{2}\right)^{2}}, \\
L_{1}\left(-\alpha \Lambda+A_{1}\right)=\varpi L_{1}, \quad L_{2}\left(\alpha \Lambda+A_{2}\right)=-\varpi L_{2}, \quad \varpi=\frac{a_{1}^{2}-a_{2}^{2}}{4-\left(a_{1}+a_{2}\right)^{1}},
\end{gathered}
$$

$$
\begin{aligned}
& \iint_{x<y}\left\langle L_{1},-\Lambda\left|z_{1}\right|_{x}+A_{1}\left|z_{1}(x)\right|-\left(a_{1}(x)-a_{1}\right) R\left|z_{1}(x)\right|\right\rangle\left\langle L_{2},\left|z_{2}(y)\right|\right\rangle e^{-\alpha(x-y)} d x d y \\
& \leq \iint_{x<y}\left\langle-\alpha L_{1} \Lambda+A_{1},\left|z_{1}\right|\right\rangle\left\langle L_{2},\left|z_{2}(y)\right|\right\rangle e^{-\alpha(x-y)} d x d y-\int_{\mathbb{R}}\left\langle L_{1} \Lambda,\left|z_{1}\right|\right\rangle\left\langle L_{2},\left|z_{2}\right|\right\rangle d y \\
& =\iint_{x<y} \varpi\left\langle L_{1},\left|z_{1}\right|\right\rangle\left\langle L_{2},\left|z_{2}(y)\right|\right\rangle e^{-\alpha(x-y)} d x d y-\int_{\mathbb{R}}\left\langle L_{1} \Lambda,\left|z_{1}\right|\right\rangle\left\langle L_{2},\left|z_{2}\right|\right\rangle d y,
\end{aligned}
$$

and similarly

$$
\begin{aligned}
& \iint_{x<y}\left\langle L_{1},\left|z_{1}(x)\right|\right\rangle\left\langle L_{2},-\Lambda\left|z_{2}\right|_{y}+A_{2}\left|z_{2}(y)\right|-\left(a_{2}(t, y)-a_{2}\right) R\left|z_{2}(y)\right|\right\rangle e^{-\alpha(x-y)} d x d y \\
& =-\iint_{x<y} \varpi\left\langle L_{1},\left|z_{1}\right|\right\rangle\left\langle L_{2},\left|z_{2}(y)\right|\right\rangle e^{-\alpha(x-y)} d x d y+\int_{\mathbb{R}}\left\langle L_{1},\left|z_{1}\right|\right\rangle\left\langle L_{2} \Lambda,\left|z_{2}\right|\right\rangle d y,
\end{aligned}
$$

We adding the integrals, only the boundary terms survives, which can be written as

$$
\int_{\mathbb{R}}\left(\frac{\left(1-a_{1}\right)\left(1+a_{2}\right)}{4-\left(a_{1}+a_{2}\right)^{2}}\left|z_{1}^{+}\right|\left|z_{2}^{-}\right|-\frac{\left(1+a_{1}\right)\left(1-a_{2}\right)}{4-\left(a_{1}+a_{2}\right)^{2}}\left|z_{1}^{-}\right|\left|z_{2}^{+}\right|\right) d x
$$

For the part with $x>y$ one obtains by the integration by parts that only the boundary terms remain, i.e.

$$
\int_{\mathbb{R}}\left|z_{1}^{-}\right|\left|z_{2}^{+}\right|-\left|z_{1}^{+}\right|\left|z_{2}^{-}\right| d x
$$


Adding the two above results, and taking into account the coefficients in front of the intergals of (6.14), we obtain

$$
\frac{d \tilde{Q}}{d t} \leq-\int_{\mathbb{R}}\left|z_{1}^{-}\right|\left|z_{2}^{+}\right|+\left|z_{1}^{+}\right|\left|z_{2}^{-}\right| d x+C \delta_{0} \int_{\mathbb{R}}\left|s_{1}\right|+\left|s_{2}\right| d x .
$$

By means of (6.11) we thus conclude that

$$
\int_{0}^{T} \int_{\mathbb{R}}\left|z_{1}^{-}(t, x) z_{2}^{+}(t, x)\right|+\left|z_{1}^{+}(t, x) z_{2}^{-}(t, x)\right| d x \leq C \delta_{0}^{2},
$$

if $\delta_{0}$ is sufficiently small. The constant $C$ collects some $c^{-1}$, where $c$ is the separation of speeds.

We now estimate also the terms $\left|z_{1}^{-} z_{2}^{-}\right|,\left|z_{1}^{+} z_{2}^{+}\right|$. We only consider the first term, since the second one is similar. We have

$$
\begin{aligned}
\left(\left|z_{1}^{-} z_{2}^{-}\right|\right)_{t}-\left(\left|z_{1}^{-} z_{2}^{-}\right|\right)_{x} \leq & \left(-\frac{1+a_{1}}{2}\left|z_{1}^{-}\right|+\frac{1-a_{1}}{2}\left|z_{1}^{+}\right|+\left|s_{1}^{-}(t, x)\right|\right)\left|z_{2}^{-}\right| \\
& +\left|z_{1}^{-}\right|\left(-\frac{1+a_{2}}{2}\left|z_{2}^{-}\right|+\frac{1-a_{2}}{2}\left|z_{2}^{+}\right|+\left|s_{2}^{-}(t, x)\right|\right)
\end{aligned}
$$

so that by means of $(6.11)$

$$
\frac{d}{d t} \int_{\mathbb{R}}\left|z_{1}^{-} z_{2}^{-}\right| d x \leq-c \int_{\mathbb{R}}\left|z_{1}^{-} z_{2}^{-}\right| d x+\int_{\mathbb{R}}\left|z_{1}^{-} z_{2}^{+}\right|+\left|z_{1}^{+} z_{2}^{-}\right| d x+C \delta_{0}^{2} \int_{\mathbb{R}}\left|s_{1}^{-}\right|+\left|s_{2}^{-}\right| d x
$$

This gives the estimates

$$
\begin{aligned}
\int_{\mathbb{R}}\left|z_{1}^{-}(t) z_{2}^{-}(t)\right| d x \leq & \int_{0}^{t} e^{c(\tau-t)} \int_{\mathbb{R}}\left|z_{1}^{-}(\tau) z_{2}^{+}(\tau)\right|+\left|z_{1}^{+}(\tau) z_{2}^{-}(\tau)\right| d x \\
& +C \delta_{0}^{2} \int_{0}^{t} e^{c(\tau-t) / 2} \int_{\mathbb{R}}\left|s_{1}^{-}(\tau)\right|+\left|s_{2}^{-}(\tau)\right| d x
\end{aligned}
$$

which integrated in time and using (6.18) yields

$$
\int_{0}^{T} \int_{\mathbb{R}}\left|z_{1}^{-}(t) z_{2}^{-}(t)\right| d x \leq C \delta_{0}^{2}
$$

Repeating the computations for $\left|z_{1}^{+} z_{2}^{+}\right|$we obtain also

$$
\int_{0}^{T} \int_{\mathbb{R}}\left|z_{1}^{+}(t) z_{2}^{+}(t)\right| d x \leq C \delta_{0}^{2} .
$$

With the estimates (6.18), (6.19), (6.20) we have computed the transversal terms appearing in the source term,

$$
\sum_{j \neq k} \int_{0}^{T} \int_{\mathbb{R}}\left(\left|h_{j}^{-}\right|+\left|h_{j}^{+}\right|\right)\left(\left|f_{k}^{-}\right|+\left|f_{k}^{+}\right|+\left|g_{k}^{-}\right|+\left|g_{k}^{+}\right|\right) d x d t \leq C \delta_{0}^{2},
$$

with $C$ sufficiently large.

6.3. Interaction terms of the same family. The terms due to interactions of waves of the same family are divided into 2 categories: non transversal terms, to which it corresponds a Glimm type functional, and energy type terms. The Glimm type interaction functional is the kinetic analog of the functional introduced in [6] to analyze the interaction terms of the same family. However in this case its construction is slightly more complicated.

The energy terms appears only due to the fact that the cut off function $\theta$ is acting, and the speed of a perturbation and the travelling wave are very different. We have seen that this also implies the ratio $f_{j}^{+} / f_{j}^{-}$to be far from 1 . 
6.3.1. Glimm functional. Before constructing the Glimm type functional for relaxation, we recall its construction for a scalar nonlinear parabolic equation

$$
u_{t}+\lambda(u) u_{x}=u_{x x} .
$$

One can in fact introduce the vector valued function $P=\left(u, f(u)-u_{x}\right)$, which satisfies the equation

$$
P_{t}+\lambda(u) P_{x}=P_{x x} .
$$

For the above equation one can show that the functional

$$
Q(t)=\frac{1}{2} \iint_{x<y}\left|P_{x}(t, x) \wedge P_{x}(t, y)\right| d x d y
$$

is decreasing: precisely

$$
\frac{d Q}{d t} \leq-\int_{\mathbb{R}}\left|P_{t}(t, x) \wedge P_{x}(t, x)\right| d x=-\int_{\mathbb{R}}\left|P_{x x}(t, x) \wedge P_{x}(t, x)\right| d x
$$

The last equation can be thought as the instantaneous are swept by the curve $P(t, x)$.

It is possible to give another interpretation of the previous functional. In fact one can consider the variable

which satisfies

$$
\tilde{p}(t, x, y)=P_{x}(t, x) \wedge P_{x}(t, y)=u_{x}(t, x) u_{t}(t, y)-u_{x}(t, y) u_{x}(t, x)
$$

Due to the symmetry $\tilde{p}(t, x, y)+p(t, y, x)=0$, the above scalar 2 -d equation can be considered in the half plane $x<y$ with boundary data $\tilde{p}(t, x, x)=0$. The functional $Q$ is now half of the $L^{1}$ norm of $\tilde{p}$, and its time derivative is the flux of $\tilde{p}$ across the boundary $\{x=y\}$.

While the first interpretation as a shortening curve is difficult to extend to relaxation, the last interpretation is more suitable: we will thus associate a $4 \times 42$-d kinetic system to a $2 \times 2$ scheme (or more precisely to $22 \times 2$ schemes) and study the flux across a boundary.

We thus consider two couple of variables $\left(z_{1}^{-}, z_{1}^{+}\right),\left(z_{2}^{-}, z_{2}^{+}\right)$, which satisfy the same $2 \times 2$ kinetic scheme, with some source term, i.e.

$$
\left\{\begin{array}{l}
z_{i, t}^{-}-z_{i, x}^{-}=-\frac{1+a(t, x)}{2} z_{i}^{-}+\frac{1-a(t, x)}{2} z_{i}^{+}+s_{i}^{-}(t, x) \\
z_{i, t}^{+}+z_{i, x}^{+}=\frac{1+a(t, x)}{2} z_{i}^{-}-\frac{1-a(t, x)}{2} z_{i}^{+}+s_{i}^{+}(t, x)
\end{array} \quad i=1,2,\right.
$$

with the standard non degeneracy assumptions

$$
|a(t, x)| \leq 1-c, c>0, \quad \int_{0}^{t} \int_{\mathbb{R}}\left|s_{1}^{ \pm}\right|+\left|s_{2}^{ \pm}\right| d x d t \leq 2 \delta_{0} .
$$

We then consider the vector valued variables $P^{-}=\left(z_{1}^{-}, z_{2}^{-}\right), P^{+}=\left(z_{1}^{+}, z_{2}^{+}\right)$, which satisfy the same equation as $z_{i}^{ \pm}$but vector valued:

$$
\left\{\begin{array}{l}
P_{t}^{-}-P_{x}^{-}=-\frac{1+a(t, x)}{2} P^{-}+\frac{1-a(t, x)}{2} P^{+}+s^{-}(t, x) \\
P_{t}^{+}+P_{x}^{+}=\frac{1+a(t, x)}{2} P^{-}-\frac{1-a(t, x)}{2} P_{i}^{+}+s^{+}(t, x)
\end{array}\right.
$$

with source terms

$$
s^{-}(t, x)=\left(\begin{array}{c}
s_{1}^{-}(t, x) \\
s_{2}^{-}(t, x)
\end{array}\right), \quad s^{+}(t, x)=\left(\begin{array}{c}
s_{1}^{+}(t, x) \\
s_{2}^{+}(t, x)
\end{array}\right) .
$$

In the vanishing viscosity case we have only one scalar variable defined on $\mathbb{R}^{2}$, while now we need to consider 4 variables,

$$
\begin{array}{ll}
P^{--}(t, x, y)=P^{-}(t, x) \wedge P^{-}(t, y), & P^{-+}(t, x, y)=P^{-}(t, x) \wedge P^{+}(t, y), \\
P^{+-}(t, x, y)=P^{+}(t, x) \wedge P^{-}(t, y), & P^{++}(t, x, y)=P^{+}(t, x) \wedge P^{+}(t, y),
\end{array}
$$

with the relations

$$
P^{--}(t, y, x)=-P^{--}(t, x, y), \quad P^{++}(t, y, x)=-P^{++}(t, x, y), \quad P^{-+}(t, y, x)+P^{+-}(t, x, y)=0
$$


The kinetic scheme these variables satisfy is clearly (for shortness denote $a(t, x)$ by $a(x)$ )

$$
\left\{\begin{array}{l}
P_{t}^{--}-P_{x}^{--}-P_{y}^{--}=\frac{1-a(x)}{2} P^{+-}+\frac{1-a(y)}{2} P^{-+}-\left(1+\frac{a(x)+a(y)}{2}\right) P^{--}+s^{--}(t, x, y) \\
P_{t}^{-+}-P_{x}^{-+}+P_{y}^{-+}=\frac{1+a(y)}{2} P^{--}+\frac{1-a(x)}{2} P^{++}-\left(1+\frac{a(x)-a(y)}{2}\right) P^{-+}+s^{-+}(t, x, y) \\
P_{t}^{+-}+P_{x}^{+-}-P_{y}^{+-}=\frac{1+a(x)}{2} P^{--}+\frac{1-a(y)}{2} P^{++}-\left(1-\frac{a(x)-a(y)}{2}\right) P^{+-}+s^{+-}(t, x, y) \\
P_{t}^{++}+P_{x}^{++}+P_{y}^{++}=\frac{1+a(y)}{2} P^{+-}+\frac{1+a(x)}{2} P^{-+}-\left(1-\frac{a(x)+a(y)}{2}\right) P^{++}+s^{++}(t, x, y)
\end{array}\right.
$$

where the source terms are

$$
s^{\alpha \beta}=\left(\begin{array}{c}
s^{\alpha}(x) \\
s^{\alpha}(x)
\end{array}\right) \wedge P^{\beta}(y)+P^{\alpha}(x) \wedge\left(\begin{array}{c}
s^{\beta}(y) \\
s^{\beta}(y)
\end{array}\right), \quad \int_{0}^{T} \int_{\mathbb{R}^{2}}\left|s^{\alpha \beta}(t, x, y)\right| d x d y d t \leq C \delta_{0}^{2} .
$$

As we notice for the viscosity case, due to the symmetries (6.29) we can consider the above system in the half plane $x \geq y$, with boundary data given by

$$
P^{--}(t, x, x)=P^{++}(t, x, x)=0, \quad P^{-+}(t, x, x)+P^{+-}(t, x, x)=0,
$$

We can describe the above boundary conditions by saying that a particle with speed $(-1,1)$ hits the boundary and bounce back with the opposite sign, or equivalently that the "conservative" variable $P^{--}+$ $P^{-+}+P^{+-}+P^{++}$is 0 at the boundary $\{x=y\}$.

Our goal is to estimate the flux of $u$ through the boundary, more precisely the number of particle which hit the line $x=y$,

$$
\int_{0}^{T} \int_{\mathbb{R}}\left|P^{-+}(t, x, x)\right| d x=\int_{0}^{T} \int_{\mathbb{R}}\left|z_{1}^{-}(t, x) z_{2}^{+}(t, x)-z_{1}^{+}(t, x) z_{2}^{-}(t, x)\right| d x d t .
$$

By setting $s^{\alpha \beta}=0$ for $t \geq T$, we can extend the solution to all $t$ and thus estimate the integral in $[0, \infty)$. The main problem in this estimate is that we cannot suppose the solution to be positive: the boundary condition (6.31) in fact changes the sign of the solution.

We thus construct the solution $P$ to $(6.30)$ as a sum of various part. It the boundary were no present, then we just obtain functions whose sum is of the order of the initial $L^{1}$ norm, due to conservation (easy to check in $\mathbb{R}^{2}$ ) and neglecting the source terms $s^{\alpha \beta}$. The presence of the boundary generates a step in this process in which some fraction of the initial $L^{1}$ mass disappears.

To understand better the construction and the final estimate, we consider here the following 1-d example.

Example 6.2. We consider the simple model

$$
\left\{\begin{array}{c}
z_{t}^{-}-z_{x}^{-}=\frac{z^{+}-z^{-}}{2} \\
z_{t}^{+}+z_{x}^{+}=\frac{z^{-}-z^{+}}{2}
\end{array}\right.
$$

in $x \geq 0$ with boundary data $f^{-}(t, 0)+f^{+}(t, 0)=0$. Our goal is to estimate

$$
\int_{0}^{+\infty}\left|z^{-}(t, 0)\right| d t
$$

To have a better control of the solution, we first notice that if the boundary data is $z^{+}(t, 0)=0$, then clearly by $L^{1}$ contraction

$$
\frac{d}{d t} \int_{\mathbb{R}^{+}}\left|z^{-}(t, x)\right|+\left|z^{+}(t, x)\right| d x \leq-\left|z^{-}(t, 0)\right|,
$$

so that the integral (6.33) is bounded by the initial $L^{1}$ norm of $z$. The above estimate just tells that the number of particle which cross the boundary (and disappears) is bounded by the total number of particles in $x>0$.

We thus have only to consider the case of an initial $\delta$ function in $f^{+}$located at $x=0$. We now decompose the solution to (6.32) with initial data $z^{-}(0, x)=0, z^{+}(0, x)=\delta(x)$ as the sum of functions $z^{ \pm, i}, i=0,1, \ldots$, each one satisfying

$$
\left\{\begin{array}{c}
z_{t}^{-, i+1}-z_{x}^{-, i+1}=\frac{z^{-, i}+z^{+, i}}{2}-z^{-, i+1} \\
z_{t}^{+, i+1}+z_{x}^{+, i+1}=\frac{z^{-, i}+z^{+, i}}{2}-z^{+, i+1}
\end{array}\right.
$$


with $z^{ \pm,-1}=0, z^{-, 0}=0, z^{+, 0}=\delta(x)$, and null initial data for $i=1,2, \ldots$ Roughly speaking, we can imagine that each function $z^{ \pm, i}$ describes a generation of particles, moving with speed \pm 1 , and with average decay time of 1 . When 2 particles of the $i$ generation decays, then 2 particles of the $i+1$ generation are created, with speed -1 and +1 .

It is simple to construct the first solutions: in fact,

$$
\begin{gathered}
z^{-, 0}(t, x)=0, \quad z^{+, 0}(t, x)=e^{-t} \delta(x-t), \\
z^{-, 1}(t, x)=\frac{e^{-t}}{2} \chi\{0 \leq x \leq t\}, \quad z^{+, 1}(t, x)=-\frac{e^{-t}}{2} \chi\{0 \leq x \leq t\}+\frac{t}{2} e^{-t} \delta(x-t) .
\end{gathered}
$$

At this point we can observe that at the next step the total source $z^{-, 1}+z^{+, 1}$ has become $1 / 2$, while the total flux of these solutions across the boundary is $1 / 2$. We thus have proved the following: after $1+1 / 2$ crossing ( 1 is due to the initial absorbing boundary), we have that $1 / 2$ of the initial $L^{1}$ norm disappear. It is thus clear that the total crossing is bounded by

$$
\frac{1+1 / 2}{1-1 / 2}=3
$$

We thus conclude with the estimate

$$
\int_{0}^{+\infty}\left|z^{-}(t, 0)\right| d t \leq 3 \int_{\mathbb{R}^{+}}\left|z^{-}(0, x)\right|+\left|z^{+}(0, x)\right| d x .
$$

In the following computations we just extend the analysis of the example to our 2-d case. Clearly also in this case we have only to study the initial data

$$
P^{+-}=\delta(x, y), \quad P^{--}=P^{-+}=P^{++}=0 .
$$

We have just to remember that the total mass flowing with absorbing boundary conditions is now

$$
\sum_{\alpha \beta} \iint_{x>y}\left|P^{\alpha \beta}(0, x, y)\right| d x d y+\sum_{\alpha \beta} \int_{0}^{T} \iint_{x>y}\left|s^{\alpha \beta}(t, x, y)\right| d x d y d t=C \delta_{0}^{2} .
$$

Here and in the following for shortness we use the indexes $\alpha, \beta=-,+$. Let $\bar{a}$ be a constant and assume that

$$
|a(t, x)-\bar{a}| \leq C \delta_{0} .
$$

This condition is satisfied if we replace $a(t, x), \bar{a}$ with $\tilde{\lambda}_{i}, \lambda_{i}(0)$.

In the 2 -d case we need to consider 4 functions. We define the $P_{1}$ as the solution of

$$
P_{1, t}^{\alpha \beta}+(\alpha, \beta) \cdot \nabla P_{1}^{\alpha \beta}=-P_{1}^{\alpha \beta},
$$

i.e.

$$
P_{1}^{+-}=e^{-t} \delta(x-t, y+t), \quad P_{1}^{++}=P_{1}^{-+}=P_{1}^{--}=0 .
$$

In this case the boundary conditions do not enter in the computation.

The function $P_{2}^{\alpha \beta}$ is defined by

$$
\left\{\begin{array}{l}
P_{2, t}^{--}-(1,1) \cdot \nabla P_{2}^{--}=\frac{1-\bar{a}}{2} e^{-t} \delta(x-t, y+t)-(1+\bar{a}) P_{2}^{--} \\
P_{2, t}^{++}+(1,1) \cdot \nabla P_{2}^{++}=\frac{1+\bar{a}}{2} e^{-t} \delta(x-t, y+t)-(1-\bar{a}) P_{2}^{++},
\end{array}\right.
$$

Also in this case the boundary data does not enter in the solution. The solution is thus

$$
\left\{\begin{array}{l}
P_{2}^{--}=\frac{1-\bar{a}}{4} e^{-(x+t) / 2-(1+\bar{a})(t-x) / 2} \chi_{[-t, t]}(x) \delta(y+t) \\
P_{2}^{++}=\frac{1+\bar{a}}{4} e^{-(t-y) / 2-(1-\bar{a})(t+y) / 2} \delta(x-t) \chi_{[-t, t]}(y)
\end{array}\right.
$$

Its total mass (i.e. its integral in $\left.\mathbb{R}^{+} \times\{x>y\}\right)$ is $\left(1+a^{2}\right) /\left(1-a^{2}\right)$.

The functions $P_{3}^{-+}, P_{3}^{+-}$are given by solving the system

$$
\left\{\begin{array}{l}
P_{3, t}^{-+}+(-1,1) \cdot \nabla P_{3}^{-+}=\frac{1+\bar{a}}{2} P_{2}^{--}+\frac{1-\bar{a}}{2} P_{2}^{++}-P_{3}^{-+} \\
P_{3, t}^{+-}+(1,-1) \cdot \nabla P_{3}^{+-}=\frac{1+\bar{a}}{2} P_{2}^{--}+\frac{1-\bar{a}}{2} P_{2}^{++}-P_{3}^{+-}
\end{array}\right.
$$


where the boundary conditions are $P_{3}^{+-}+P_{3}^{-+}=0$. The solution is

$$
\begin{aligned}
P_{3}^{-+}= & \frac{1-\bar{a}^{2}}{16} e^{-t+\bar{a}(x+y) / 2} \chi\{x \in[-t, t], y \in[-t, t], x+y \leq 0\} \\
P_{3}^{+-}= & -\frac{1-\bar{a}^{2}}{16} e^{-t+\bar{a}(x+y) / 2} \chi\{x \in[-t, t], y \in[-t, t], x+y \leq 0\} \\
& +\frac{1-\bar{a}^{2}}{16}(t+x) e^{-(x+t) / 2-(1+\bar{a})(t-x) / 2} \chi_{[-t, t]}(x) \delta(y+t) \\
& +\frac{1-\bar{a}^{2}}{16}(t-y) e^{-(t-y) / 2-(1-\bar{a})(t+y) / 2} \delta(x-t) \chi_{[-t, t]}(y) .
\end{aligned}
$$

Note that the total mass entering into $P_{3}^{-+}$is now $1 / 2$. The flux of $P_{3}^{-+}$across the boundary is $1 / 8$. The mass of the sum $P_{3}^{-+}+P_{3}^{-+}$is due to the last singular part, and its value is $1 / 2$.

Finally the function $P_{4}$ satisfies (6.30) with source terms the errors we have neglected, which are

$$
P_{3}^{-+}+P_{3}^{+-}, \quad(a-\bar{a})\left(\left|P_{1}^{+-}\right|+\left|P_{1}^{--}\right|+\left|P_{2}^{++}\right|+\left|P_{3}^{-+}\right|+\left|P_{3}^{+-}\right|\right) .
$$

Clearly these errors are of order $1+C \delta_{0}$, because of cancellations in the sum $P_{3}^{-+}+P_{3}^{+-}$, the exponential decay of $P_{i}^{\alpha \beta}$ and (6.37).

As in Example 6.2, we have thus that the total crossing is for the special initial data is

$$
\frac{1+\text { flux of the our approximate solutions }}{1-\text { mass disappearing }}=\frac{1+1 / 8}{1-\left(1 / 2-C \delta_{0}\right)} \leq 3\left(1+C \delta_{0}\right) \leq 4,
$$

if $\delta_{0}$ is sufficiently small, and hence we have the estimate

$$
\int_{0}^{T} \int_{\mathbb{R}}\left|P^{-+}(t, x, x)\right| d x d t=\int_{0}^{T} \int_{\mathbb{R}}\left|z_{1}^{-}(t, x) z_{2}^{+}(t, x)-z_{1}^{+}(t, x) z_{2}^{-}(t, x)\right| d x d t \leq C \delta_{0}^{2} .
$$

The above estimate can be used for all the terms

$$
\left|f_{j}^{-} g_{j}^{+}-f_{j}^{+} g_{j}^{-}\right|, \quad\left|f_{j}^{-} h_{j}^{+}-f_{j}^{+} h_{j}^{-}\right|, \quad\left|h_{j}^{-} g_{j}^{+}-h_{j}^{+} g_{j}^{-}\right|, \quad\left|h_{j}^{-} \iota_{j}^{+}-h_{j}^{+} \iota_{j}^{-}\right|,
$$

yielding the desired estimates $\leq C \delta_{0}^{2}$. It can be used also for $\left|f_{j}^{-} \iota_{j}^{+}-f_{j}^{+} \iota_{j}^{-}\right|,\left|g_{j}^{-} \iota_{j}^{+}-g_{j}^{+} \iota_{j}^{-}\right|$, but we will not need them (they are needed when studying $\iota$, however).

6.3.2. Energy estimates around a solution. We are left with the estimates of the terms $h_{j}^{+}+h_{j}^{-}$in the regions where they are far from the equilibrium $\left(1-\tilde{\lambda}^{-}, 1+\tilde{\lambda}_{j}^{-}\right)$. In this regions, the non linearity can be controlled, and thus we have only to estimate the energy for a linear equation. What is interesting is that we will also obtain an energy type functional around travelling profiles, which in practice tells us that the equilibrium is given by $\left(f_{j}^{-}, f_{j}^{+}\right) \simeq(1-\sigma, 1+\sigma)$, where $\sigma$ is the speed of the local travelling wave. This in some sense extends the energy estimate also to the non linear travelling profiles.

Consider as before the two systems (6.25), with $a(t, x)-\bar{a}=\mathcal{O}(1) \delta_{0}$, and introduce the functional

$$
\begin{aligned}
E & \doteq \int_{\mathbb{R}}(1+\bar{a})\left(z_{1}^{-}\right)^{2} \psi\left(z_{2}^{-} / z_{1}^{-}\right)+(1-\bar{a})\left(z_{1}^{+}\right)^{2} \psi\left(z_{1}^{+} / z_{2}^{+}\right) \\
& =\int_{\mathbb{R}}(1+\bar{a})\left(z_{1}^{-}\right)^{2} \psi^{-}+(1-\bar{a})\left(z_{1}^{+}\right)^{2} \psi^{+}
\end{aligned}
$$

where $\psi$ is a cut off function of the form

$$
\psi(x)= \begin{cases}0 & |x| \leq \delta_{2} / 2 \\ \text { smooth connection } & \delta_{2} / 2<|x|<\delta_{2} \quad|\theta| \leq 1 . \\ 1 & |x| \geq \delta_{2}\end{cases}
$$

Differentiating w.r.t. $t$ we have

$$
\begin{aligned}
\frac{d E}{d t}= & -\int_{\mathbb{R}}\left((1+\bar{a}) z_{1}^{-} \psi^{-}-(1-\bar{a}) z_{1}^{+} \psi^{+}\right)\left((1+a) z_{1}^{-}-(1-a) z_{1}^{+}\right) \\
& +2 \int_{\mathbb{R}}\left((1+\bar{a})\left(\psi^{-}\right)^{\prime}-(1-\bar{a})\left(\psi^{+}\right)^{\prime}\right)\left(z_{1}^{-} z_{2}^{+}-z_{1}^{+} z_{2}^{-}\right)+C \delta_{0}^{2} \int_{\mathbb{R}}\left|s_{1}^{ \pm}\right|+\left|s_{2}^{ \pm}\right| d x .
\end{aligned}
$$

The last term is only the interactions of waves of the same family we studied at the previous section. 
We now make the following additional assumptions: $z_{1}^{ \pm}$and $z_{2}^{ \pm}$are tied with the same relation among $\iota$ and $h$, i.e.

$$
z_{2}^{-}+z_{2}^{+}=(1+\bar{a}) z_{1}^{-}-(1-\bar{a}) z_{1}^{+}+\varsigma(t, x)
$$

where $\varsigma$ is assumed to be integrable and of order $2 \delta_{0}$.

As we did in studying the source terms, we simplify the first integral by considering the various situations can occur:

(1) if $z_{1}^{-} z_{1}^{+}<0$, then we can bound the right hand side of (6.41) by the decrease of the total variation, i.e. we have

$$
\frac{d E}{d t} \leq C \delta_{0}^{2} \int_{z_{1}^{-} z_{1}^{+}<0}\left|z_{1}^{-}\right|+\left|z_{1}^{+}\right| d x+C \delta_{0}^{2} \int_{\mathbb{R}}\left|s_{1}^{ \pm}\right|+\left|s_{2}^{ \pm}\right| d x .
$$

(2) if $\left|z_{2}^{ \pm} / z_{1}^{ \pm}\right| \geq \delta_{2} / 2$, then we obtain that by similar computations as the one used in Section 5.4 and using (5.9)

$$
\begin{aligned}
(1+a) z_{1}^{-}-(1-a) z_{1}^{+} & =(1+\bar{a}) z_{1}^{-}-(1-\bar{a}) z_{1}^{+}+(a-\bar{a})\left(z_{1}^{-}+z_{1}^{+}\right) \\
& =z_{2}^{-}+z_{2}^{+}+(a-\bar{a})\left(z_{1}^{-}+z_{1}^{+}\right)+\varsigma(t, x) \\
& =\left(1+(a-\bar{a}) z_{1}^{-} / z_{2}^{-}\right) z_{2}^{-}+\left(1+(a-\bar{a}) z_{1}^{+} / z_{2}^{+}\right) z_{2}^{+}+\varsigma(t, x) .
\end{aligned}
$$

Substituting the above equation into the first term of the right hand side of (6.41) we have thus

$$
\begin{aligned}
& \left|\left((1+\bar{a}) z_{1}^{-} \psi^{-}-(1-\bar{a}) z_{1}^{+} \psi^{+}\right)\left((1+a) z_{1}^{-}-(1-a) z_{1}^{+}\right)\right| \\
& \quad \geq c \min \left\{\psi^{-}, \psi^{+}\right\}\left|z_{2}^{-}+z_{2}^{+}\right|\left|\left(1+(a-\bar{a}) z_{1}^{-} / z_{2}^{-}\right) z_{2}^{-}+\left(1+(a-\bar{a}) z_{1}^{+} / z_{2}^{+}\right) z_{2}^{+}\right|-C\left|z_{2}^{-}+z_{2}^{+}\right| \varsigma(t, x) .
\end{aligned}
$$

If $z_{2}^{-} z_{2}^{+}<0$, then we can again use the $L^{1}$ norm decay of $z_{2}^{ \pm}$. Otherwise, we obtain a term of the form

We thus have that

$$
-c \min \left\{\psi^{-}, \psi^{+}\right\}\left(z_{2}^{-}+z_{2}^{+}\right)^{2}+C \delta_{0}^{2} \varsigma(t, x) .
$$

$$
\begin{aligned}
\frac{d E}{d t} \leq & -c \int_{\mathbb{R}} \min \left\{\psi^{-}, \psi^{+}\right\}\left(z_{2}^{-}+z_{2}^{+}\right)^{2}+C \delta_{0}^{2} \int_{z_{1}^{-} z_{1}^{+}<0}\left|z_{1}^{-}\right|+\left|z_{1}^{+}\right| d x \\
& +C \int_{\mathbb{R}}\left|z_{1}^{-} z_{2}^{+}-z_{1}^{+} z_{2}^{-}\right| d x+C \delta_{0}^{2} \int_{\mathbb{R}}\left(\left|s_{1}^{ \pm}\right|+\left|s_{2}^{ \pm}\right|+|\varsigma|\right) d x,
\end{aligned}
$$

from which we conclude, using (6.38) that

$$
\int_{0}^{T} \int_{\left|z_{2}^{ \pm} / z_{1}^{ \pm}\right| \geq \delta_{2} / 2}\left(z_{2}^{-}+z_{2}^{+}\right)^{2} \leq C \delta_{0}^{2} .
$$

To obtain the estimate for $z_{1}^{ \pm}$, observe that similarly to the cases studied in Section 5.4 one has

$$
\left(z_{2}^{-}+z_{2}^{+}\right) \chi\left\{\left|z_{2}^{ \pm} / z_{1}^{ \pm}\right| \geq \delta_{2} / 2\right\} \simeq C^{-1}\left(z_{1}^{-}+z_{1}^{+}\right),
$$

so that we have also the estimates for $z_{1}^{ \pm}$. Finally observe that when $\left|z_{2}^{ \pm} / z_{1}^{ \pm}\right| \geq \delta_{2} / 2$, then

$$
\left(\frac{z_{1}^{+}}{z_{1}^{-}}-\frac{1+a}{1+a}\right) \notin\left[-5 \delta_{2} / 4,5 \delta_{2} / 4\right], \quad\left(\frac{z_{2}^{+}}{z_{2}^{-}}-\frac{1+a}{1+a}\right) \notin\left[-5 \delta_{2} / 4,5 \delta_{2} / 4\right] .
$$

With this we have estimated the energy terms appearing in the source (5.31), i.e.

$$
\begin{aligned}
& C \sum_{j}\left(\left|f_{j}^{-}+f_{j}^{+}\right|^{2}+\left|g_{j}^{-}+g_{j}^{+}\right|^{2}\right) \chi\left\{\left(\frac{f_{j}^{+}}{f_{j}^{-}}-\frac{1+\lambda_{j}(0)}{1+\lambda_{j}(0)}\right) \notin\left[-5 \delta_{2} / 4,5 \delta_{2} / 4\right]\right\} \\
& +C \sum_{j}\left|h_{j}^{-}+h_{j}^{+}\right|^{2} \chi\left\{\left(\frac{h_{j}^{+}}{h_{j}^{-}}-\frac{1+\lambda_{j}(0)}{1+\lambda_{j}(0)}\right) \notin\left[-5 \delta_{2} / 4,5 \delta_{2} / 4\right]\right\} .
\end{aligned}
$$

Note that for the estimate of $h$ we have supposed to have written the equations for $\iota$ and estimated the source terms. 
To conclude the section, we extend the energy estimate to travelling profiles: to determine the equilibrium, we can use the speed $\sigma$ instead of $\lambda_{i}(0)$. It is in fact straightforward that on a travelling wave of the $j$-th family

One has

$$
\frac{f^{-}}{1-\sigma}=\frac{f^{+}}{1+\sigma} \quad \Longleftrightarrow(1+\sigma) f_{j}^{-}=(1-\sigma) f_{j}^{+}
$$

$$
\begin{aligned}
\frac{d}{d t} \frac{1}{2} \int \frac{\left(f_{j}^{-}\right)^{2}}{1-\sigma_{j}^{-}}+\frac{\left(f_{j}^{+}\right)^{2}}{1+\sigma_{j}^{+}} d x= & \int\left(\frac{f_{j}^{-}}{1-\sigma_{j}^{-}}-\frac{f_{j}^{+}}{1+\sigma_{j}^{+}}\right)\left(\frac{1-\tilde{\lambda}_{j}}{2} f_{j}^{+}-\frac{1+\tilde{\lambda}_{j}}{2} f_{j}^{-}\right) d x+\int s(t, x) d x \\
= & -\int \frac{\left(1+\sigma_{j}^{+}\right)\left(1-\sigma_{j}^{-}\right)}{2}\left(\frac{f_{j}^{-}}{1-\sigma_{j}^{-}}-\frac{f_{j}^{+}}{1+\sigma_{j}^{+}}\right)^{2} d x+\int s(t, x) d x \\
= & -\int \frac{\left(1+\sigma_{j}^{+}\right)\left(1-\sigma_{j}^{-}\right)}{2}\left(\frac{f_{j}^{-}}{1-\sigma_{j}^{-}}-\frac{f_{j}^{+}}{1+\sigma_{j}^{+}}\right)^{2} d x+\int s(t, x) d x \\
= & -\int \frac{1}{2\left(1+\sigma_{j}^{+}\right)\left(1-\sigma_{j}^{-}\right)}\left(g_{j}^{-}+\theta_{j}^{-} f_{j}^{-}+g_{j}^{+}+\theta_{j}^{+} f_{j}^{+}\right)^{2} d x \\
& +\mathcal{O}(1) \delta_{0} \int\left(\left|f_{j}^{+}\right|+f_{j}^{-} \mid\right)\left|g_{j}^{-}+\theta_{j}^{-} f_{j}^{-}+g_{j}^{+}+\theta_{j}^{+} f_{j}^{+}\right| d x+\int s(t, x) d x,
\end{aligned}
$$

where we have used the relations among $f$ and $g$, and for shortness we collect all the source terms we know to estimate in the function $s(t, x)$.

Integrating in $t$ we obtain that $g_{j}^{-}+\sigma_{j}^{-} f_{j}^{-}+g_{j}^{+}+\sigma_{j}^{+} f_{j}^{+} \rightarrow 0$, so that using again (5.7) is follows that $\sigma_{j}^{-}-\sigma_{j}^{+} \rightarrow 0$ and $\left(1+\sigma_{j}\right) f_{j}^{-}-\left(1-\sigma_{j}\right) f_{j}^{+} \rightarrow 0$. Moreover we have the estimate

$$
\int_{0}^{T} \int_{\mathbb{R}}\left(\left(1+\sigma_{j}^{+}\right) f_{j}^{-}-\left(1-\sigma_{j}^{-}\right) f_{j}^{+}\right)^{2} d x d t \leq C \delta_{0}^{2} .
$$

This inequality can also be obtained by

$$
\left(1+\sigma_{j}^{-}\right) f_{j}^{-}-\left(1-\sigma_{j}^{+}\right) f_{j}^{+}=C\left(\left|g_{j}^{-}+\theta_{j}^{-} f_{j}^{-}\right|+\left|g_{j}^{+}+\theta_{j}^{+} f_{j}^{+}\right|+\left(\left|f_{j}^{+}\right|+f_{j}^{-} \mid\right)\left|\theta_{j}^{-}-\theta_{j}^{+}\right|\right)
$$

and using the results of Section 6 .

The above estimate is the nonlinear counterpart of the standard estimate

$$
\int_{0}^{T} \int_{\mathbb{R}}\left((1+\lambda) f^{-}-(1-\lambda) f^{+}\right)^{2} d x d t=\int_{\mathbb{R}}(1+\lambda)\left(f^{-}\right)^{2}+(1-\lambda)\left(f^{+}\right)^{2} d x .
$$

for linear $2 \times 2$ systems with average speed $\lambda$.

\section{Conclusion of the Proof of TheOrem 1.1}

Since we have proved that the $L^{1}([0, T] \times \mathbb{R})$ norm of the source term is quadratic w.r.t. the $L^{1}$ norm of the solution, using the argument outlined in Section 5.4 we have that all the couple of kinetic variables $\left(f^{-}, f^{+}\right),\left(g^{-}, g^{+}\right),\left(h^{-}, h^{+}\right),\left(\iota^{-}, \iota^{+}\right)$have bounded $L^{1}$ norm uniformly in time. If we observe that the initial data enters in the computation multiplied by a constant $C$, then we can assume $\delta_{1} \leq C^{-1} \delta_{0}$.

Of all these variables, only $\left(f^{-}, f^{+}\right),\left(h^{-}, h^{+}\right)$are meaningful: the first ones are related with $\left(u_{x}, u_{t}\right)$, the second with the perturbation $h$ of $u$. The other variables are used in the travelling wave decomposition. If the system where in conservation form, also $\left(g^{-}, g^{+}\right)$and $\left(\iota^{-}, \iota^{+}\right)$can be described as derivatives and perturbations of the variables $\left(F^{-}, F^{+}\right)$.

We can reassume the results of the above section in the following proposition:

Proposition 7.1. Let the initial data $\left(u_{0}, u_{0, t}\right)$ and $\left(h_{0}, h_{0, t}\right)$ be smooth and satisfy $(2.8),(2.9)$, substituting $u_{x}$ with $h$. Then the variable $\tilde{u}(t, x)=u(t, x)+e^{-t} u_{t, 0}(x)$ satisfies

$$
\left\|\tilde{u}_{x}(t)\right\|_{L^{1}},\left\|\tilde{u}_{t}(t)\right\|_{L^{1}} \leq C \delta_{0}
$$

In a similar way, the variable $\tilde{h}(t, x)=h(t, x)+e^{-t} h_{t, 0}(x)$ satisfies

$$
\|\tilde{h}(t)\|_{L^{1}},\left\|\tilde{h}_{t}(t)\right\|_{L^{1}} \leq L\left(\left\|h_{0}+h_{t, 0}\right\|_{L^{1}}+C\left(\left\|h_{0, t x}\right\|_{L^{1}}+\left\|h_{0, t x x}\right\|_{L^{1}}\right)\right) .
$$

for some constant $L$. 
Proof. We have only to show how the regularity estimates yield $\left\|\tilde{h}_{t}(t)\right\|_{L^{1}} \leq C \delta_{0}$. In fact $h$ satisfies a linear equation, so that the $\delta_{0}$ can be replaced with $C\|h\|_{L^{1}}$.

From the results of Theorem 3.1 in Section 3 we obtain a uniform estimate for $\tilde{\iota}_{x}$, and the result follows by using the first equation of (2.38).

In the above proposition we estimate $h_{t}$ because $\iota$ has no direct relation with $u$ if the system is not conservative and the initial data in $u_{t}$ are not in $L^{1}$, while $\tilde{h}_{t}$ can be clearly interpreted as the perturbation of $\tilde{u}_{t}$.

The final computation is the estimate on the Lipschitz dependence, and this follows by a homotopy argument. One consider two initial data $\left(u_{0}, u_{0, t}\right),\left(\hat{u}_{0}, \hat{u}_{0, t}\right)$, with

$$
\left\|u_{0}-\hat{u}_{0}\right\|_{L^{1}},\left\|\hat{u}_{0, t}-\hat{u}_{0, t}\right\|_{L^{1}} \leq \delta_{1} \text {. }
$$

and consider the path $\left(u_{0}^{\theta}, u_{0, t}^{\theta}\right), \theta \in[0,1]$, connecting $\left(u_{0}, u_{0, t}\right)$ to $\left(\hat{u}_{0}, \hat{u}_{0, t}\right)$ defined by

$$
\left(u_{0}^{\theta}, u_{0, t}^{\theta}\right)=\left((1-\theta) u_{0}+\theta \hat{u}_{0},(1-\theta) u_{0, t}+\theta \hat{u}_{0, t}\right) .
$$

Clearly, each $\left(u_{0}^{\theta}, u_{t, 0}^{\theta}\right)$ has uniform bounded total variation, so that it can be considered as a starting point of the flow $S_{t}$ of $(2.1)$, and one has

$$
\left(u(t), u_{t}(t)\right)-\left(\hat{u}(t), \hat{u}_{t}(t)\right)=S_{t}\left(u_{0}, u_{0, t}\right)-S_{t}\left(\hat{u}_{0}, \hat{u}_{0, t}\right)=\int_{0}^{1} \frac{d}{d \theta} S_{t}\left(u_{0}^{\theta}, u_{0, t}^{\theta}\right) d \theta .
$$

Since $d S_{t}\left(u^{\theta}, v^{\theta}\right) / d t$ is the evolution of the linearized equation along the solution $\left(u^{\theta}(t), u_{t}^{\theta}(t)\right)=S_{t}\left(u_{0}^{\theta}, u_{0, t}^{\theta}\right)$ with initial data $\left(u_{0}-\hat{u}_{0}, u_{0, t}-\hat{u}_{0, t}\right)$, we can apply (7.2) of Proposition 7.1 to obtain the estimate

$$
\begin{aligned}
\left\|u(t)-\hat{u}(t)+e^{-t}\left(u_{0, t}-\hat{u}_{0, t}\right)\right\|_{L^{1}} & +\left\|u_{t}(t)-\hat{u}_{t}(t)-e^{-t}\left(u_{0, t}-\hat{u}_{0, t}\right)\right\|_{L^{1}} \\
\leq & L \int_{0}^{1}\left\|\left(u(0)+u_{0, t}\right)-\left(\hat{u}(0)+\hat{u}_{0, t}\right)\right\|_{L^{1}} d \theta \\
& +L C \int_{0}^{1}\left(\left\|u_{0, t x}-\hat{u}_{0, t x}\right\|_{L^{1}}+\left\|u_{0, t x x}-\hat{u}_{0, t x x}\right\|_{L^{1}}\right) d \theta .
\end{aligned}
$$

We thus have proved (1.8), and this concludes the proof of Theorem 1.1.

\section{The hyperbolic Limit $\epsilon \rightarrow 0$}

We first rewrite Theorem 1.1 after the hyperbolic rescaling $(t, x) \mapsto(\epsilon t, \epsilon x)$ :

Theorem 8.1. Consider the equation

$$
u_{t}+A(u) u_{x}=\epsilon\left(u_{x x}-u_{t t}\right) \text {, }
$$

with $A(u)$ strictly hyperbolic and with eigenvalues in $[-1+c, 1-c], c>0$. Assume that the initial data $\left(u_{0}, u_{0, t}\right)$ satisfy

$$
\begin{gathered}
\left\|u_{0}\right\|_{L^{\infty}},\left\|\epsilon u_{0, t}\right\|_{L^{\infty}},\left\|u_{0, x}\right\|_{L^{1}},\left\|\epsilon u_{0, t x}\right\|_{L^{1}} \leq \delta_{1} \\
\left\|\epsilon u_{0, x x}\right\|_{L^{1}},\left\|\epsilon^{2} u_{0, t x x}\right\|_{L^{1}},\left\|\epsilon^{2} u_{0, x x x}\right\|_{L^{1}},\left\|\epsilon^{3} u_{0, t x x x}\right\|_{L^{1}} \leq C \delta_{1} .
\end{gathered}
$$

If $\delta_{1} \leq C^{-1} \delta_{0}$, with $C$ sufficiently large, then there exists a solution $\left(u, u_{t}\right)$ defined for all $t \geq 0$, and with uniformly bounded $L^{1}$ norm in both $u_{x}, \epsilon u_{t x}$, and depending continuously w.r.t. the initial data: for some constant $L$ independent on $\epsilon$

$$
\left\|u(t)+\epsilon e^{-t / \epsilon} u_{0, t}-\left(\hat{u}(s)+\epsilon e^{-s / \epsilon} \hat{u}_{0, t}\right)\right\|_{L^{1}}+\left\|\left(u_{t}(t)-e^{-t / \epsilon} u_{0, t}\right)-\left(\hat{u}_{t}(s)-e^{-s / \epsilon} \hat{u}_{0, t}\right)\right\|_{L^{1}}
$$

$$
\leq L\left(|t-s|+\left\|\left(u_{0}+\epsilon u_{0, t}\right)-\left(\hat{u}_{0}+\epsilon \hat{u}_{0, t}\right)\right\|_{L^{1}}+\epsilon^{2}\left\|u_{0, t x}-\hat{u}_{0, t x}\right\|_{L^{1}}+\epsilon^{3}\left\|u_{0, t x x}-\hat{u}_{0, t x x}\right\|_{L^{1}}\right) .
$$

Since we are considering the hyperbolic limit $\epsilon \rightarrow 0$, we have to assume the convergence of the initial data: fixed two functions $u_{0}, u_{0, t}$, we assume that the initial data for the case $\epsilon \neq 0$ are

$$
u_{0}^{\epsilon}(x)=u_{0}(x), \quad u_{0, t}^{\epsilon}=\frac{1}{\epsilon} u_{0, t}(x) .
$$


Passing to the limit $\epsilon \rightarrow 0$, recalling (8.2) and taking a subsequence $\epsilon_{i}$ such that $u^{\epsilon_{i}}$ converges to some $u$ on a dense countable set $\left\{t_{i}\right\}_{i \in \mathbb{N}}$ of times, we have for all $t, s>0$ that $u$ satisfies

$$
\|u(t)-\hat{u}(s)\|_{L^{1}} \leq L\left(|t-s|+\left\|\left(u_{0}+u_{0, t}\right)-\left(\hat{u}_{0}+\hat{u}_{0, t}\right)\right\|_{L^{1}}\right) .
$$

Since for $\tau>0$ we have that

$$
u^{\epsilon}(\tau)+\epsilon u_{t}^{\epsilon}(\tau)=u^{\epsilon}(\tau)+\epsilon \tilde{u}_{t}^{\epsilon}(\tau)+e^{-t / \epsilon} u_{t, 0}(\tau) \quad \longrightarrow u(\tau)
$$

as $\epsilon \rightarrow 0$, then we have also for $0<\tau \leq t, s$

$$
\|u(t)-\hat{u}(s)\|_{L^{1}} \leq L\left(|t-s|+\|u(\tau)-\hat{u}(\tau)\|_{L^{1}}\right)
$$

The Lipschitz dependence w.r.t. $t$ implies the convergence for all $t \in \mathbb{R}^{+}$. Moreover we have that as $t \rightarrow 0$ the limiting function $u(t)$ tends to the initial data $u_{0}+u_{t, 0}$.

Note that we have a Lipschitz dependence on the initial data, and the domain of functions satisfying (8.2) becomes dense in the set

$$
D=\left\{u, u_{t}: \text { Tot.Var. }(u), \text { Tot.Var. }\left(u_{t}\right) \leq \delta_{1}\right\},
$$

as $\epsilon \rightarrow 0$. Thus we can extend by continuity the Lipschitz flow $\mathcal{S}_{t}$ to all the initial data $u_{0}+u_{t, 0}$ in $D$.

By defining $\mathcal{D}$ to be the evolution of $D$ under the flow $\mathcal{S}_{t}$, then we obtain a Lipschitz continuous semigroup. In principle this semigroup may depend on the limiting sequence considered: the final result of this section is to prove that it is independent on the limiting sequence, hence the whole sequence $u^{\epsilon}(t)$ converges to $\mathcal{S}_{t}\left(u_{0}+u_{0, t}\right)$ for $\epsilon \rightarrow 0$.

Remark 8.2. Clearly, up to subsequences, one has also

$$
u_{t}^{\epsilon} \rightarrow\left(\mathcal{S}_{t}\left(u_{0}+u_{0, t}\right)\right)_{t}, \quad u_{x}^{\epsilon} \rightarrow\left(\mathcal{S}_{t}\left(u_{0}+u_{0, t}\right)\right)_{x},
$$

weakly in measure. One can also define the non conservative product $A(u) u_{x}$ in terms of $-u_{t}$, i.e. for a.e. $t$, to each jump $\left(u^{-}, u^{+}\right)$in $\bar{x}$ there corresponds a shock wave with speed $\sigma$ and

$$
A(u) u_{x}=-\sigma\left(u^{+}-u^{-}\right) \delta(x-\bar{x}) .
$$

These results however do not yield additional information on $\mathcal{S}_{t}$. See the regularity results of [9].

8.0.3. Solution to a Riemann problem. A particular solution of the limiting semigroup is the trajectory of $\mathcal{S}$ with initial data

$$
\bar{u}(0, x)= \begin{cases}u^{-} & x \leq 0 \\ u^{+} & x>0\end{cases}
$$

with $u^{-}, u^{+}$close to $0,\left|u^{-}-u^{+}\right|$small. For this special initial data there is a general technique to determine which is the limiting solution by studying the evolution equation on the center manifold (4.5). In $[4,5]$ it is shown that, under the assumptions of uniform BV estimates, finite speed of propagation and $L^{1}$ stability, this limit does not depend on the approximating sequence, and in the conservative case it coincides with the Riemann Solver constructed by the vanishing viscosity limit. The results of the previous sections show that our system satisfies all the needed hypotheses.

More precisely, if $\tilde{r}_{i}, \tilde{\lambda}_{i}$ are the functions defined in (4.3), (4.6) respectively, then consider the system in $\mathbb{R}^{n+2}$,

$$
\left\{\begin{array}{rcc}
u(\tau) & = & u^{-}+\int_{0}^{\tau} \tilde{r}_{i}\left(u(\varsigma), v_{i}(\varsigma), \sigma_{i}(\varsigma)\right) d \varsigma \\
v_{i}(\tau) & = & \left(f_{i}\left(\tau ; u, v_{i}, \sigma_{i}\right)-\operatorname{conv}_{[0, s]} f_{i}\left(\tau ; u, v_{i}, \sigma_{i}\right)\right) /\left(1-\sigma_{i}(\tau)^{2}\right) \\
\sigma_{i}(\tau) & = & \frac{d}{d \tau} \operatorname{conv}_{[0, s]} f_{i}\left(\tau ; u, v_{i}, \sigma_{i}\right)
\end{array}\right.
$$

where we define the "reduced" flux $f_{i}$ by

$$
f_{i}\left(\tau ; u, v_{i}, \sigma_{i}\right) \doteq \int_{0}^{\tau} \tilde{\lambda}_{i}\left(u(\varsigma), v_{i}(\varsigma), \sigma_{i}(\varsigma)\right) d \varsigma,
$$

and $\operatorname{conv}_{[0, s]} f_{i}$ denotes the convex envelope of $f_{i}$ in $[0, s]$. 
In [4] it is shown that for all $u^{-}$close to 0 and $s$ sufficiently small, there exists a curve $\gamma_{i}:[0, s] \mapsto \mathbb{R}^{n+2}$, solution of the above system starting from $u^{-}$and taking values near $\left(u^{-}, 0, \lambda_{i}\left(u^{-}\right)\right)$. Moreover by construction the speed $\sigma_{i}$ is increasing, so that we can define the (piecewise constant) function $u(\xi)$ as

$$
u_{i}(\xi)=u\left(\sigma^{-1}(\xi)\right) .
$$

Recall that the above two functions are the components of the curve $\gamma_{i}$, i.e. $\gamma_{i}(s)=\left(u(s), v_{i}(s), \sigma_{i}(s)\right)$.

Define the reduced curve $\mathcal{T}_{i}\left(s, u^{-}\right)$as the end point of $(\gamma(s))_{u}$, and consider the composed map

$$
\left(s_{1}, \ldots, s_{n}\right) \mapsto \mathcal{T}_{n}\left(s_{n}, \mathcal{T}_{n-1}\left(s_{n-1}, \mathcal{T}_{n-2}\left(s_{n-2}, \ldots \mathcal{T}_{1}\left(s_{1}, u\right)\right)\right)\right) .
$$

In [4] it is shown that the above map is invertible if $u^{-},\left(s_{1}, \ldots, s_{n}\right)$ are close to 0 . Fixed thus $u^{-}, u^{+}$, we can find the $n$ curves $\gamma_{i}$ connecting $u^{-}$to $u^{+}$.

The solution to the Riemann (8.7) is then given by piecing together the functions $u_{i}(\xi)$ defined by (8.10) for each curve $\gamma_{i}$ obtained by inverting (8.11). Since the speed $\sigma_{i}$ are close to $\lambda_{i}(0)$, by the strict hyperbolicity these functions do not overlap.

8.0.4. Viscosity solutions of $u_{t}+A(u) u_{x}=0$. We recall that a Viscosity Solution of a quasilinear hyperbolic system

$$
u_{t}+A(u) u_{x}=0
$$

is defined as follows.

Let $u(t, x)$ be a BV function w.r.t. $x$. Given a point $(\tau, \xi)$, denote by $U_{(u ; \tau, \xi)}^{\sharp}$ the solution to the Riemann problem

$$
u(\tau, x)= \begin{cases}\lim _{y \rightarrow \xi-} u(\tau, y) & x \leq \xi \\ \lim _{y \rightarrow \xi+} u(\tau, y) & x>\xi\end{cases}
$$

This solution is obtained by the Riemann solver defined in [4], i.e., it is the unique limit of $u^{\epsilon}(t)$ with the special initial data (8.13).

We denote by $U_{(u ; \tau, \xi)}^{b}$ the solution to the linear system

$$
u_{t}+A(u(\tau, \xi)) u_{x}=0
$$

with initial data $u(\tau, x)$.

A Viscosity Solution to (8.12) is now a function $u(t, x)$ satisfying the integral estimates:

(i) At every point $(\tau, \xi)$, for some $\beta^{\prime}>0$

$$
\lim _{h \rightarrow 0^{+}} \frac{1}{h} \int_{\xi-h \beta}^{\xi+h \beta}\left|u(\tau+h, x)-U_{(u ; \tau, \xi)}^{\sharp}(\tau+h, x)\right| d x=0 .
$$

(ii) There are constant $C, \beta \leq \beta^{\prime}$ such that for every $a<\xi<b$

$$
\lim _{h \rightarrow 0^{+}} \frac{1}{h} \int_{a+h \beta}^{b-h \beta}\left|u(\tau+h, x)-U_{(u ; \tau, \xi)}^{b}(\tau+h, x)\right| d x \leq C \mathcal{V}(u ;] a, b[)^{2} .
$$

8.1. Uniqueness of the limiting semigroup. The last step in proving uniqueness is to thus to show that any limit of a convergent subsequence is a Viscosity Solution to

$$
u_{t}+A(u) u_{x}=0
$$

in the sense of [8]. In fact it follows that any trajectory is a trajectory of the semigroup $\mathcal{S}$, i.e., the limit does not depend on the subsequence.

Using the same technique of [5], one can prove the following Lemma:

Lemma 8.3. Let $\mathcal{S}:[0, \infty[\times \mathcal{D} \mapsto \mathcal{D}$ be a semigroup of solutions, constructed as the limit of a sequence $u^{\epsilon_{i}}$ of the wave equation (8.1) and defined on a domain $\mathcal{D} \subset L_{\mathrm{loc}}^{1}$ of functions with small total variation. Let $u:[0, T] \mapsto \mathcal{D}$ be Lipschitz continuous w.r.t. time, i.e.

$$
\|u(t)-u(s)\|_{L^{1}} \leq L|t-s|
$$


for some constant $L$ and all $s, t \in[0, T]$. Then

$$
u(t)=\mathcal{S}_{t} u(0) \quad \forall t \in[0, T]
$$

if and only if $u$ is a viscosity solution of (8.12).

Proof. The first result follows by $L^{1}$ stability and the results of Section 8.0.3. In fact, we can consider two initial data $u_{0}, \hat{u}_{0}$, where $u_{0}$ is $u(\tau)$ restricted to $[\xi-2 h, \xi+2 h]$ and $\hat{u}$ the exact Riemann problem (8.13). Then we have

$$
\frac{1}{h} \int_{\xi-h \beta}^{\xi+h \beta}\left|u(\tau+h, x)-U_{(u ; \tau, \xi)}^{\sharp}(\tau+h, x)\right| d x \leq L \frac{1}{h} \int_{\xi-2 h}^{\xi+2 h}\left|u_{0}(y)-\hat{u}(y)\right| d y=o(h),
$$

because $u_{0}$ is $\mathrm{BV}$ and because of (8.13).

To prove (8.16), we have directly that for $\epsilon>0$

$$
u_{t}+A(u(\tau, \xi)) u_{x}=(A(u(\tau, \xi))-A(u)) u_{x}+\epsilon\left(u_{x x}-u_{t t}\right),
$$

and by regularity estimates, neglecting the exponentially decaying term,

$$
\epsilon\left\|u_{x x}-u_{t t}\right\|_{L^{1}},\left\|(A(u(\tau, \xi))-A(u)) u_{x}\right\|_{L^{1}} \leq C \operatorname{Tot} . \operatorname{Var} .(u)^{2} .
$$

The result thus follows by using the finite speed of propagation for (8.1) and the following lemma on Lipschitz semigroups [8]:

Lemma 8.4. If $S:[0, T] \times \mathcal{D} \mapsto \mathcal{D}$ is a Lipschitz semigroup on a domain $\mathcal{D} \subset E, E$ Banach space, and satisfying

$$
\left\|S_{t} u-S_{s} v\right\|_{E} \leq L\left(|t-s|+\|u-v\|_{E}\right), \quad \forall u, v \in \mathcal{D},
$$

and $w:[0, T] \mapsto \mathcal{D}$ is Lipschitz continuous, then we have the estimate

$$
\left\|w(T)-S_{t} w(0)\right\|_{E} \leq L \int_{0}^{T} \liminf _{\tau \rightarrow 0} \frac{\left\|w(t+\tau)-S_{\tau} w(t)\right\|_{E}}{\tau} d t .
$$

In fact, the linear equation on the left hand side of (8.19) is clearly a Lipschitz semigroup, the $L^{1}$ norm of the right hand side is the infinitesimal time error, so that we have

$$
\int_{a+h}^{b-h}\left|u(\tau+h, x)-U_{(u ; \tau, \xi)}^{b}(\tau+h, x)\right| d x \leq L \int_{0}^{h} \operatorname{Tot.Var} .(u ;] a, b[)^{2} \leq L h \operatorname{Tot} . \operatorname{Var} .(u ;] a, b[)^{2} .
$$

In particular $\mathcal{S}_{t} u$ is a viscosity solution to (8.12). This concludes the proof of uniqueness of the limit, and Theorem 1.2 is proved.

\section{Appendix A. Explicit computations of the source terms for the Perturbation $h$}

Here we compute the source terms for the equations of the components of the perturbation $h$. We have for the right hand side of $(2.39)$

$$
\begin{aligned}
h_{t}^{-}-h_{x}^{-}= & \sum_{j}\left(\left(h_{j, t}^{-}-h_{j, x}^{-}\right) \tilde{r}_{j}^{-}+h_{j}^{-}\left(f_{j, t}^{-}-f_{j, x}^{-}\right)\left(\tilde{r}_{j, f}^{-}+\theta_{j, f}^{-} \tilde{r}_{j, \sigma}^{-}\right)+h_{j}^{-}\left(g_{j, t}^{-}-g_{j, x}^{-}\right) \theta_{j, g}^{-} \tilde{r}_{j, \sigma}^{-}\right) \\
& +\sum_{j k} h_{j}^{-}\left(g_{k}^{-}-\left(1+\lambda_{k}(0)\right) f_{k}^{-}\right) D \tilde{r}_{j}^{-} \tilde{r}_{k}^{-}+\sum_{j k} h_{j}^{-}\left(g_{k}^{+}-\left(1+\lambda_{k}(0)\right) f_{k}^{+}\right) D \tilde{r}_{j}^{-} \tilde{r}_{k}^{+} \\
& +\sum_{j} h_{j}^{-} e^{-t} D \tilde{r}_{j}^{-} u_{t}(0),
\end{aligned}
$$




$$
\begin{aligned}
h_{t}^{+}+h_{x}^{+}= & \sum_{j}\left(\left(h_{j, t}^{+}-h_{j, x}^{+}\right) \tilde{r}_{j}^{+}+h_{j}^{+}\left(f_{j, t}^{+}-f_{j, x}^{+}\right)\left(\tilde{r}_{j, f}^{+}+\theta_{j, f}^{+} \tilde{r}_{j, \sigma}^{+}\right)+h_{j}^{+}\left(g_{j, t}^{+}-g_{j, x}^{+}\right) \theta_{j, g}^{+} \tilde{r}_{j, \sigma}^{+}\right) \\
& +\sum_{j k} h_{j}^{+}\left(g_{k}^{-}+\left(1-\lambda_{j}(0)\right) f_{k}^{-}\right) D \tilde{r}_{j}^{+} \tilde{r}_{k}^{-}+\sum_{j k} h_{j}^{+}\left(g_{k}^{+}+\left(1-\lambda_{j}(0)\right) f_{k}^{+}\right) D \tilde{r}_{j}^{+} \tilde{r}_{k}^{+} \\
& +\sum_{j} h_{j}^{+} e^{-t} D \tilde{r}_{j}^{+} u_{t}(0),
\end{aligned}
$$

Using now

$$
\begin{gathered}
D A \tilde{r}_{i}^{ \pm}=\tilde{\lambda}_{i}^{ \pm} \tilde{r}_{i}^{ \pm}+\left(\tilde{\lambda}_{i}^{ \pm}-\sigma\right) f_{i}^{ \pm} \tilde{r}_{i, f}^{ \pm}+(1 \mp \sigma) f_{i}^{ \pm} D \tilde{r}_{i}^{ \pm} \tilde{r}_{i}^{ \pm} \\
\tilde{r}_{i, f}^{+}=\frac{1}{1 \pm \sigma_{i}^{ \pm}} \tilde{r}_{i, v}\left(u, f_{i}^{ \pm} /\left(1 \pm \sigma_{i}^{ \pm}\right), \sigma_{i}^{ \pm}\right)
\end{gathered}
$$

we can compute

$$
\begin{aligned}
& \frac{I+D A(u)}{2} \tilde{r}_{j}^{-}\left(u, f_{j}^{-}, \sigma_{j}^{-}\right)=\frac{1+\tilde{\lambda}_{j}^{-}}{2} \tilde{r}_{j}^{-}+\frac{\tilde{\lambda}_{j}^{-}-\sigma_{j}^{-}}{2} \frac{f_{j}^{-}}{1-\sigma_{j}^{-}} \tilde{r}_{j, v}^{-}+\frac{1+\sigma_{j}^{-}}{2} f_{j}^{-} D \tilde{r}_{j}^{-} \tilde{r}_{j}^{-}, \\
& \frac{I-D A(u)}{2} \tilde{r}_{j}^{+}\left(u, f_{j}^{+}, \sigma_{j}^{+}\right)=\frac{1+\tilde{\lambda}_{j}^{+}}{2} \tilde{r}_{j}^{+}-\frac{\tilde{\lambda}_{j}^{+}-\sigma_{j}^{+}}{2} \frac{f_{j}^{+}}{1+\sigma_{j}^{+}} \tilde{r}_{j, v}^{+}-\frac{1-\sigma_{j}^{+}}{2} f_{j}^{+} D \tilde{r}_{j}^{+} \tilde{r}_{j}^{+} .
\end{aligned}
$$

We thus obtain that the system for $\left(h^{-}, h^{+}\right)$can be rewritten as

$$
\begin{aligned}
& \sum_{j}\left(h_{j, t}^{-}-h_{j, x}^{-}+\frac{1+\tilde{\lambda}_{j}^{-}}{2} h_{j}^{-}-\frac{1-\tilde{\lambda}_{j}^{+}}{2} h_{j}^{+}\right) \tilde{r}_{j}^{-} \\
& +\sum_{j} h_{j}^{-}\left(f_{j, t}^{-}-f_{j, x}^{-}+\frac{1+\tilde{\lambda}_{j}^{-}}{2} f_{j}^{-}-\frac{1-\tilde{\lambda}_{j}^{+}}{2} f_{j}^{+}\right)\left(\tilde{r}_{j, v}^{-} /\left(1-\sigma_{j}^{-}\right)+\theta_{j, f}^{-} \tilde{r}_{j, \sigma}^{-}\right) \\
& +\sum_{j} h_{j}^{-}\left(g_{j, t}^{-}-g_{j, x}^{-}+\frac{1+\tilde{\lambda}_{j}^{-}}{2} g_{j}^{-}-\frac{1-\tilde{\lambda}_{j}^{+}}{2} g_{j}^{+}\right) \theta_{j, g}^{-} \tilde{r}_{j, \sigma}^{-}=\tilde{s}_{h}^{-}(t, x), \\
& \sum_{j}\left(h_{j, t}^{+}+h_{j, x}^{+}-\frac{1+\tilde{\lambda}_{j}^{-}}{2} h_{j}^{-}+\frac{1-\tilde{\lambda}_{j}^{+}}{2} h_{j}^{+}\right) \tilde{r}_{j}^{+} \\
& +\sum_{j} h_{j}^{+}\left(f_{j, t}^{+}+f_{j, x}^{+}-\frac{1+\tilde{\lambda}_{j}^{-}}{2} f_{j}^{-}+\frac{1-\tilde{\lambda}_{j}^{+}}{2} f_{j}^{+}\right)\left(\tilde{r}_{j, v}^{+} /\left(1+\sigma_{j}^{+}\right)+\theta_{j, f}^{+} \tilde{r}_{j, \sigma}^{+}\right) \\
& +\sum_{j} h_{j}^{+}\left(g_{j, t}^{+}+g_{j, x}^{+}-\frac{1+\tilde{\lambda}_{j}^{-}}{2} g_{j}^{-}+\frac{1-\tilde{\lambda}_{j}^{+}}{2} g_{j}^{+}\right) \theta_{j, g}^{+} \tilde{r}_{j, \sigma}^{+}=\tilde{s}_{h}^{+}(t, x),
\end{aligned}
$$

where the source terms are

$$
\begin{aligned}
\tilde{s}_{h}^{-}(t, x)= & -\sum_{j \neq k} h_{j}^{-}\left(g_{k}^{-}-f_{k}^{-}\right) D \tilde{r}_{j}^{-} \tilde{r}_{k}^{-}+\sum_{j \neq k} h_{j}^{-}\left(g_{k}^{+}-f_{k}^{+}\right) D \tilde{r}_{j}^{-} \tilde{r}_{k}^{+} \\
& -\sum_{j} h_{j}^{-}\left[\left(g_{j}^{-}-\left(1+\bar{\lambda}_{j}\right) f_{j}^{-}\right) D \tilde{r}_{j}^{-} \tilde{r}_{j}^{-}+\frac{1+\sigma_{j}^{-}}{2} f_{j}^{-} D \tilde{r}_{j}^{-} \tilde{r}_{j}^{-}+\left(g_{j}^{+}-\left(1+\bar{\lambda}_{j}\right) f_{j}^{+}\right) D \tilde{r}_{j}^{-} \tilde{r}_{j}^{+}-\frac{1-\sigma_{j}^{+}}{2} f_{j}^{+} D \tilde{r}_{j}^{+} r_{j}^{+}\right] \\
& +\sum_{j} h_{j}^{-}\left(\frac{1-\tilde{\lambda}_{j}^{+}}{2} f_{j}^{+}-\frac{1+\tilde{\lambda}_{j}^{-}}{2} f_{j}^{-}+\frac{\tilde{\lambda}_{j}^{-}-\sigma_{j}^{-}}{2} f_{j}^{-}\right) \frac{\tilde{r}_{j, v}^{-}}{1-\sigma_{j}^{-}}+\sum_{j} h_{j}^{+} \frac{\tilde{\lambda}_{j}^{+}-\sigma_{j}^{+}}{2} \frac{f_{j}^{+}}{1+\sigma_{j}^{+}} \tilde{r}_{j, v}^{+} \\
& +\sum_{j} h_{j}^{-}\left(\frac{1+\tilde{\lambda}_{j}^{+}}{2}\right)\left[g_{j}^{+}-\frac{g_{j}^{-}}{f_{j}^{-}} f_{j}^{+}\right]\left(\theta_{j}^{-}\right)^{\prime} \tilde{r}_{j, \sigma}^{-} / f_{j}^{-}+e^{-t} h_{0, t}(x)-\int_{0}^{t} e^{-(t-\tau)} D A\left(u_{x} \times h-h \times u_{x}\right) d \tau,
\end{aligned}
$$




$$
\begin{aligned}
\tilde{s}_{h}^{+}(t, x)= & -\sum_{j \neq k} h_{j}^{+}\left(g_{k}^{-}+f_{k}^{-}\right) D \tilde{r}_{j}^{+} \tilde{r}_{k}^{-}+\sum_{j \neq k} h_{j}^{+}\left(g_{k}^{+}+f_{k}^{+}\right) D \tilde{r}_{j}^{+} \tilde{r}_{k}^{+} \\
& -\sum_{j} h_{j}^{+}\left[\left(g_{j}^{-}-\left(1+\bar{\lambda}_{j}\right) f_{j}^{-}\right) D \tilde{r}_{j}^{+} \tilde{r}_{j}^{-}+\frac{1+\sigma_{j}^{-}}{2} f_{j}^{-} D \tilde{r}_{j}^{-} \tilde{r}_{j}^{-}+\left(g_{j}^{+}-\left(1+\bar{\lambda}_{j}\right) f_{j}^{+}\right) D \tilde{r}_{j}^{+} \tilde{r}_{j}^{+}-\frac{1-\sigma_{j}^{+}}{2} f_{j}^{+} D \tilde{r}_{j}^{+} r_{j}^{+}\right] \\
& -\sum_{j} h_{j}^{+}\left(\frac{1-\tilde{\lambda}_{j}^{+}}{2} f_{j}^{+}-\frac{1+\tilde{\lambda}_{j}^{-}}{2} f_{j}^{-}+\frac{\tilde{\lambda}_{j}^{+}-\sigma_{j}^{+}}{2} f_{j}^{+}\right) \frac{\tilde{r}_{j, v}^{+}}{1+\sigma_{j}^{-}}+\sum_{j} h_{j}^{-} \frac{\tilde{\lambda}_{j}^{-}-\sigma_{j}^{-}}{2} \frac{f_{j}^{-}}{1-\sigma_{j}^{-}} \tilde{r}_{j, v}^{-} \\
& +\sum_{j} h_{j}^{+}\left(\frac{1-\tilde{\lambda}_{j}^{+}}{2}\right)\left[g_{j}^{-}-\frac{g_{j}^{+}}{f_{j}^{+}} f_{j}^{-}\right]\left(\theta_{j}^{+}\right)^{\prime} \tilde{r}_{j, \sigma}^{+} / f_{j}^{+}+e^{-t} h_{0, t}(x)-\int_{0}^{t} e^{-(t-\tau)} D A\left(u_{x} \times h-h \times u_{x}\right) d \tau .
\end{aligned}
$$

Using now (5.7), (5.6) it follows that we have the estimates

$$
\begin{aligned}
& \left(g_{j}^{-}-\left(1+\bar{\lambda}_{j}\right) f_{j}^{-}\right) D \tilde{r}_{j}^{-} \tilde{r}_{j}^{-}+\frac{1+\sigma_{j}^{-}}{2} f_{j}^{-} D \tilde{r}_{j}^{-} \tilde{r}_{j}^{-}+\left(g_{j}^{+}-\left(1+\bar{\lambda}_{j}\right) f_{j}^{+}\right) D \tilde{r}_{j}^{-} \tilde{r}_{j}^{+}-\frac{1-\sigma_{j}^{+}}{2} f_{j}^{+} D \tilde{r}_{j}^{+} r_{j}^{+} \\
= & \left(\left(g_{j}^{-}-\left(1+\bar{\lambda}_{j}\right) f_{j}^{-}\right) D \tilde{r}_{j}^{-} \tilde{r}_{j}^{-}+\frac{1+\sigma_{j}^{-}}{2} f_{j}^{-}+\left(g_{j}^{+}-\left(1+\bar{\lambda}_{j}\right) f_{j}^{+}\right)-\frac{1-\sigma_{j}^{+}}{2} f_{j}^{+}\right) D \tilde{r}_{j}^{-} r_{j}^{-} \\
& +\mathcal{O}(1)\left(g_{j}^{-}+\theta_{j}^{-} f_{j}^{-}\right)+\mathcal{O}(1)\left(g_{j}^{+}+\theta_{j}^{+} f_{j}^{+}\right)+\mathcal{O}(1)\left(\left|f_{j}^{-}\right|+\left|f_{j}^{+}\right|\right)\left(\sigma_{j}^{+}-\sigma_{j}^{-}\right) \\
= & \mathcal{O}(1)\left(g_{j}^{-}+\theta_{j}^{-} f_{j}^{-}\right)+\mathcal{O}(1)\left(g_{j}^{+}+\theta_{j}^{+} f_{j}^{+}\right)+\mathcal{O}(1)\left(\left|f_{j}^{-}\right|+\left|f_{j}^{+}\right|\right)\left(\sigma_{j}^{+}-\sigma_{j}^{-}\right), \\
& \left(g_{j}^{-}-\left(1+\bar{\lambda}_{j}\right) f_{j}^{-}\right) D \tilde{r}_{j}^{+} \tilde{r}_{j}^{-}+\frac{1+\sigma_{j}^{-}}{2} f_{j}^{-} D \tilde{r}_{j}^{-} \tilde{r}_{j}^{-}+\left(g_{j}^{+}-\left(1+\bar{\lambda}_{j}\right) f_{j}^{+}\right) D \tilde{r}_{j}^{+} \tilde{r}_{j}^{+}-\frac{1-\sigma_{j}^{+}}{2} f_{j}^{+} D \tilde{r}_{j}^{+} r_{j}^{+} \\
= & \mathcal{O}(1)\left(g_{j}^{-}+\theta_{j}^{-} f_{j}^{-}\right)+\mathcal{O}(1)\left(g_{j}^{+}+\theta_{j}^{+} f_{j}^{+}\right)+\mathcal{O}(1)\left(\left|f_{j}^{-}\right|+\left|f_{j}^{+}\right|\right)\left(\sigma_{j}^{+}-\sigma_{j}^{-}\right), \\
h_{j}^{-} & \left(\frac{1-\tilde{\lambda}_{j}^{+}}{2} f_{j}^{+}-\frac{1+\tilde{\lambda}_{j}^{-}}{2} f_{j}^{-}+\frac{\tilde{\lambda}_{j}^{-}-\sigma_{j}^{-}}{2} f_{j}^{-}\right) \frac{\tilde{r}_{j, v}^{-}}{1-\sigma_{j}^{-}}+h_{j}^{+} \frac{\tilde{\lambda}_{j}^{+}-\sigma_{j}^{+}}{2} \frac{f_{j}^{+}}{1+\sigma_{j}^{+}} \tilde{r}_{j, v}^{+} \\
= & \mathcal{O}(1)\left(\left|h_{j}^{-}\right|+\left|h_{j}^{+}\right|\right)\left(\left|g_{j}^{-}+\theta_{j}^{-} f_{j}^{-}\right|+\left|g_{j}^{+}+\theta_{j}^{+} f_{j}^{+}\right|+\left(\left|f_{j}^{-}\right|+\left|f_{j}^{+}\right|\right)\left|\sigma_{j}^{+}-\sigma_{j}^{-}\right|\right)+\mathcal{O}(1)\left(h_{j}^{-} f_{j}^{+}-h_{j}^{+} f_{j}^{-}\right), \\
h_{j}^{+} & \left(\frac{1-\tilde{\lambda}_{j}^{+}}{2} f_{j}^{+}-\frac{1+\tilde{\lambda}_{j}^{-}}{2} f_{j}^{-}+\frac{\tilde{\lambda}_{j}^{+}-\sigma_{j}^{+}}{2} f_{j}^{+}\right) \frac{\tilde{r}_{j, v}^{+}}{1+\sigma_{j}^{-}}+h_{j}^{-} \frac{\tilde{\lambda}_{j}^{-}-\sigma_{j}^{-}}{2} \frac{f_{j}^{-}}{1-\sigma_{j}^{-}} \tilde{r}_{j, v}^{-} \\
= & \mathcal{O}(1)\left(\left|h_{j}^{-}\right|+\left|h_{j}^{+}\right|\right)\left(\left|g_{j}^{-}+\theta_{j}^{-} f_{j}^{-}\right|+\left|g_{j}^{+}+\theta_{j}^{+} f_{j}^{+}\right|+\left(\left|f_{j}^{-}\right|+\left|f_{j}^{+}\right|\right)\left|\sigma_{j}^{+}-\sigma_{j}^{-}\right|\right)+\mathcal{O}(1)\left(h_{j}^{-} f_{j}^{+}-h_{j}^{+} f_{j}^{-}\right),
\end{aligned}
$$

We thus recover the estimates (5.23).

\section{REFERENCES}

[1] P. Baiti, A. Bressan, and H.K. Jenssen. Instability of travelling profiles for the Lax-Friedrichs scheme. preprint.

[2] S. Bianchini. A Glimm type functional for a special Jin-Xin relaxation model. Ann. Inst. H. Poincarè, 18(1):19-42, 2001.

[3] S. Bianchini. BV solutions to semidiscrete schemes. Arch. Rat. Mech. Anal., 167(1):1-81, 2003.

[4] S. Bianchini. On the Riemann problem for non-conservative hyperbolic systems. Arch. Rat. Mech. Anal., 166(1):1-26, 2003.

[5] S. Bianchini and A. Bressan. Vanishing viscosity solutions of nonlinear hyperbolic systems. preprint SISSA, 2001.

[6] S. Bianchini and A. Bressan. On a Lyapunov functional relating viscous conservation laws and shortening curves. Nonlinear Analysis TMA, 51(4):649-662, 2002.

[7] S. Bianchini, B. Hanouzet, and R. Natalini. Asymptotic behavior of smooth solutions for dissipative hyperbolic systems with a convex entropy. preprint, 2004.

[8] A. Bressan. The unique limit of the Glimm scheme. Arch. Rational Mech. Anal., 130:205-230, 1995.

[9] A. Bressan. Hyperbolic systems of conservation laws. Oxford Univ. Press, 2000.

[10] A. Bressan and R.M. Colombo. The semigroup generated by $2 \times 2$ conservation laws. Arch. Rational Mech. Anal., 133:1-75, 1995.

[11] A. Bressan, G. Crasta, and B. Piccoli. Well posedness of the Cauchy problem for $n \times n$ systems of conservation laws. Memoir A.M.S., 694, 2000.

[12] A. Bressan and P. Goatin. Oleinik type estimates and uniqueness for $n \times n$ conservation laws. J. Differential Equations, 156:26-49, 1999. 
[13] A. Bressan and M. Lewicka. A uniqueness condition for hyperbolic systems of conservation laws. Discr Cont. Dyn. Systems, 6-3:673-682, 2000.

[14] A. Bressan, T.P. Liu, and T. Yang. $L^{1}$ stability estimates for $n \times n$ conservation laws. Arch. Rat. Mech. Anal., 149:1-22, 1999.

[15] A. Bressan and W. Shen. BV estimates for multicomponent chromatography with relaxation. Discrete Cont. Dynamical Systems, 48:235-277, 2000.

[16] C. Dafermos. Hyperbolic conservation laws in continuous physics. Springer, 2000.

[17] J. Glimm. Solutions in the large for nonlinear hyperbolic systems of equations. Comm. Pure Appl. Math., 18:697-715, 1965.

[18] B. Hanouzet and R. Natalini. Existence of solution for relaxation with convex entropy. Arch. Rat. Mech. Anal., 2003.

[19] S. Jin and Z. Xin. The relaxation schemes for systems of conservation laws in arbitrary space dimensions. Comm. Pure Appl. Math., 48:235-277, 1995.

[20] T.-P. Liu. Hyperbolic conservation laws with relaxation. Commun. Math. Phys., 108:153-175, 1987.

[21] R. Natalini. Convergence to equilibrium for the relaxation approximations of conservation laws. Comm. Pure Appl. Math., 49:795-823, 1998.

[22] R. Natalini. Recent results on hyperbolic relaxation problems. In H. Freistühler, editor, Analysis of systems of conservation laws, pages 128-198. Chapman \& Hall/CRC, 1998.

[23] Y. Zeng. Gas dynamics in thermal nonequilibrium and general hyperbolic systems with relaxation. Arch. Rational Mech. Anal., 150:225-279, 1999.

E-mail address: bianchini@iac.cnr.it

$U R L:$ http://www.iac.rm.cnr.it/〜bianchin/ 